\title{
Combining Bidentate Lewis Acid Catalysis and Photochemistry: Formal Insertion of $o$-Xylene Into an Enamine Double Bond
}

Sebastian Ahles, ${ }^{+, \ddagger}$ Julia Ruhl, ${ }^{\dagger, \ddagger}$ Marcel A. Strauss, ${ }^{\dagger, \neq}$ Hermann A. Wegner ${ }^{*,+, \neq}$

${ }^{+}$Institute of Organic Chemistry, Justus Liebig University Giessen, Heinrich-BuffRing 17, 35392 Giessen, Germany

${ }^{\ddagger}$ Center for Materials Research (LaMa), Justus Liebig University Giessen, Heinrich-Buff-Ring 16, 35392 Giessen, Germany

hermann.a.wegner@org.chemie.uni-giessen.de

General Information: $\quad 2$

Synthesis: $\quad 4$

Optimization of Irradiation Wavelength for the Domino IEDDA/PIRO Reaction: 23

Isomerization Monitoring of IEDDA/PIRO Product: $\quad 24$

Degradation of BDLA-Phthalazine Complex by Irradiation: 25

NMR Spectra: $\quad 26$

Computations: $\quad 56$

References: $\quad 66$ 


\section{General Information:}

Domino-IEDDA-reactions were set up in a nitrogen filled MBRAUN UNIlab glove box. Other air and/or water sensitive reactions were carried out in a fume hood under Schlenk conditions.

\section{NMR:}

NMR spectra were measured on a Bruker Avance II $200 \mathrm{MHz}$, Avance II $400 \mathrm{MHz}$, Avance III $400 \mathrm{MHz}$ $\mathrm{HD}$ or Avance III $600 \mathrm{MHz}$ spectrometer at $25{ }^{\circ} \mathrm{C}$ if not otherwise noted. The ${ }^{1} \mathrm{H}(7.26 \mathrm{ppm})$ or ${ }^{13} \mathrm{C}$ (77.16 ppm) chemical shift of internal residual $\mathrm{CHCl}_{3}$ from $\mathrm{CDCl}_{3}$ was used as reference. Boron trifluoride diethyl etherate was used for ${ }^{19} \mathrm{~F}(-153 \mathrm{ppm})$ as external reference.

\section{UV/Vis:}

Spectra were measured with a SPECORD ${ }^{\circledR} 200$ PLUS UV/Vis spectrophotometer equipped with two automatic eightfold cell changers and a Peltier thermostat system for temperature control manufactured by Analytik Jena. The spectrophotometer system was operated by the software ASpect UV from Analytik Jena. The samples were measured in QS High Precision Cells made of Quartz Suprasil ${ }^{\circledR}$ by Hellma Analytics with a light path of $10 \mathrm{~mm}$.

MS:

ESI-MS spectra were measured on a Bruker Micro TOF.

\section{Chemicals:}

The chemicals were purchased from Sigma-Aldrich, Acros Organics, Alfa Aesar and TCI Europe. Anhydrous solvents were purchased from Acros Organics.

Deuterated solvents were purchased from Euriso - Top GmbH.

Solids were dried over Sicapent ${ }^{\circledR}$ and under high vacuum if necessary.

Technical grade solvents, used during work-up and purification, were distilled prior to use.

Aldehydes 6 were purified by distillation, degassed by freeze-pump-thaw-cycles, and stored in a nitrogen filled glove box.

Amines 7 were degassed by freeze-pump-thaw-cycles, dried either by distillation over $\mathrm{CaH}_{2}$ or storage over molecular sieve $3 \AA$, and were stored in a nitrogen filled glove box.

Bidentate Lewis acid BDLA was synthesized as described in literature, and was stored in a nitrogen filled glove box. ${ }^{1,2}$

Substituted phthalazines $\mathbf{1} \mathbf{b}-\mathbf{f},{ }^{3,4}$ and aldehyde $\mathbf{6 d}^{5}$ were synthesized as described in literature.

\section{Column Chromatography:}

Flash column chromatography was carried out with Silica $60 \mathrm{M}(0.04-0.063 \mathrm{~mm})$ from MachereyNagel GmbH \& Co. KG.

Thin layer chromatography was carried out on Polygram ${ }^{\circledR} \mathrm{SIL} \mathrm{G/UV}{ }_{254}$ from Macherey-Nagel GmbH \& Co. KG.

In cases when $\mathrm{NEt}_{3}$ was added to the eluent, the TLC plates were washed with cyclohexane containing $1 \% \mathrm{NEt}_{3}$ and dried prior to use. For a good separation the correct amount of $\mathrm{NEt}_{3}$ is essential. It depends strongly on the acidity of used Silica. 
LEDs:

Following LEDs were used for irradiation:

\begin{tabular}{ccl}
$\lambda_{\max } / \mathrm{nm}$ & $\Delta \lambda_{\text {FWHM }} / \mathrm{nm}$ & Typ \\
\hline 385 & 10 & NCSU276AT-U385 \\
405 & 12 & NCSU276AT-U405 \\
$425-430$ & 14 & LHUV-0425-0650 \\
448 & 20 & LXML-PR01-0500 \\
470 & 20 & LXML-PB01-0030 \\
500 & 30 & NCSE119AT
\end{tabular}




\section{Synthesis:}

\section{General Procedure for Domino IEDDA/PIRO Reaction Catalyzed by BDLA:}<smiles>[R]#Cc1cccc2cn[nH+]cc12</smiles>

1<smiles>[R]CC(=O)[18OH]</smiles>

6<smiles>CB1c2ccccc2B(C)c2ccccc21</smiles>

BDLA<smiles>[R]/C=C\c1ccccc1/C=C/N([R])[R]</smiles>

4

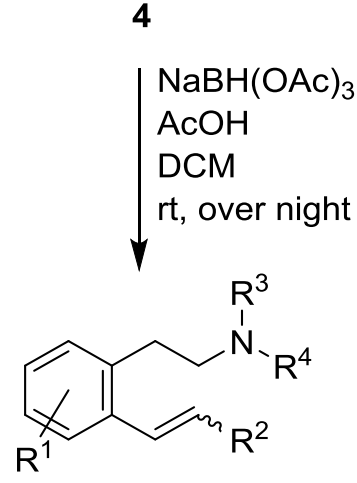

5

The reaction was set up in a nitrogen filled glovebox.

Phthalazine 1 and BDLA were suspended in THF (dry, degassed) $(2.00 \mathrm{~mL})$. Then, amine 7 was added, the reaction vessel was sealed and the remaining part of the reaction was carried out in a fume hood. The reaction mixture was heated/cooled to the reported temperature, and irradiated with a LED. Afterwards aldehyde 6 was added. Gas evolution was observed after a few minutes. For electron deficient phthalazines $\mathbf{1}$, the reaction mixture was initially cooled, and the temperature was slowly increased until gas evolution started. The reaction was kept irradiated at this temperature overnight. Then, the yellow solution was concentrated under reduced pressure $\left(10^{-1} \mathrm{mbar}\right.$, volatile compounds were condensed into a cooling trap cooled by liquid nitrogen). The remaining oil (in some cases suspension) was transferred with DCM (dry, degassed) $(3 \times 2.00 \mathrm{~mL})$ into a Schlenk tube containing $\mathrm{NaBH}(\mathrm{OAc})_{3}$. Then, $\mathrm{AcOH}$ was added. The yellow white suspension was stirred at $\mathrm{rt}$ overnight. Afterwards it was quenched by the addition of $1 \mathrm{M} \mathrm{NaOH}$ solution $(15.0 \mathrm{~mL})$. After gas evolution ceased the layers were separated. The aqueous one was extracted with DCM $(3 \times 10.0 \mathrm{~mL})$. The combined organic fractions were washed with brine $(20.0 \mathrm{~mL})$, dried over $\mathrm{Na}_{2} \mathrm{SO}_{4}$, filtered and concentrated under reduced pressure. The crude product was purified by flash column chromatography. 


\section{Phenethylamine 5a}
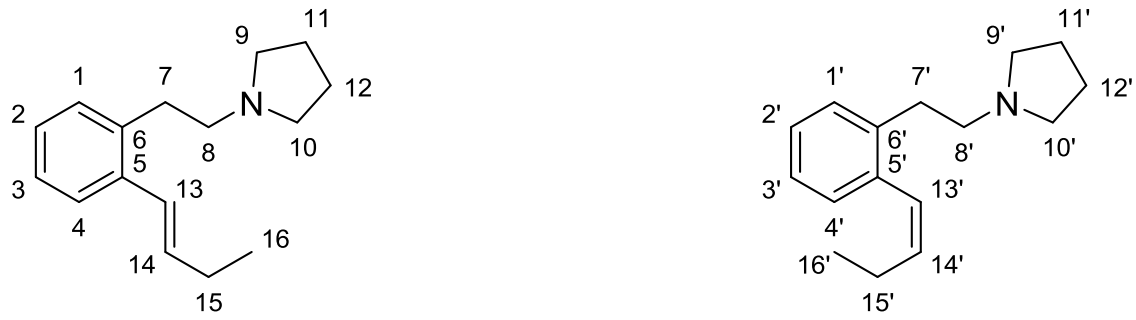

Starting materials:

Phthalazine (1a) $(98.6 \mathrm{mg}, 750 \mu \mathrm{mol}, 1.00$ equiv); BDLA (3.80 mg, $18.6 \mu \mathrm{mol}, 2.49 \mathrm{~mol} \%) ;$ pyrrolidine (7a) $\left(74.7 \mu \mathrm{L}, 900 \mu \mathrm{mol}, 1.20\right.$ equiv); butyraldehyde (6a) $\left(100 \mu \mathrm{L}, 1.09 \mathrm{mmol}, 1.45\right.$ equiv); $\mathrm{NaBH}(\mathrm{OAc})_{3}$ (328 mg, $1.50 \mathrm{mmol}, 2.00$ equiv); $\mathrm{AcOH}$ (90.4 $\mu \mathrm{L}, 1.50 \mathrm{mmol}, 2.00$ equiv).

LED:

$425-430 \mathrm{~nm}$

Temperature:

$30{ }^{\circ} \mathrm{C}$

Purification:

Flash column chromatography (2 times) $\left(\mathrm{SiO}_{2}: 20 \mathrm{~g}\right.$, cyclohexane/EtOAc $\left.+1 \% \mathrm{NEt}_{3}, 2: 1\right)$.

Yield: mixture of $E / Z$-isomers E-5a/Z-5a (1.00 : 1.78)

$139 \mathrm{mg}(604 \mu \mathrm{mol}, 81 \%) ;$ pale yellow oil.

\section{$E-5 a:$}

${ }^{1} \mathrm{H}$ NMR (400 MHz, $\mathrm{CDCl}_{3}$ with 0.03\% v/v TMS): $\delta 7.46-7.39(\mathrm{~m}, 1 \mathrm{H}, \mathrm{H} 4), 7.24-7.12(\mathrm{~m}, 3 \mathrm{H}, \mathrm{H} 1, \mathrm{H} 2$, $\mathrm{H} 3), 6.65(\mathrm{~d}, J=15.6 \mathrm{~Hz}, 1 \mathrm{H}, \mathrm{H} 13), 6.14(\mathrm{dt}, J=15.7,6.6 \mathrm{~Hz}, 1 \mathrm{H}, \mathrm{H} 14), 2.95-2.86(\mathrm{~m}, 2 \mathrm{H}, \mathrm{H} 7), 2.66-$ $2.53(\mathrm{~m}, 6 \mathrm{H}, \mathrm{H} 8-\mathrm{H} 10), 2.31-2.20(\mathrm{~m}, 4 \mathrm{H}, \mathrm{H} 15), 1.87-1.76(\mathrm{~m}, 4 \mathrm{H}, \mathrm{H} 11, \mathrm{H} 12), 1.10(\mathrm{t}, J=7.5 \mathrm{~Hz}, 3 \mathrm{H}$, H16).

${ }^{13} \mathrm{C}\left\{{ }^{1} \mathrm{H}\right\}$ NMR (101 MHz, $\mathrm{CDCl}_{3}$ with $0.03 \% \mathrm{v} / \mathrm{v}$ TMS): $\delta 137.3$ (C6), 137.0 (C5), 134.6 (C14), 129.9 (C1), 127.1 (C3), 126.6 (C4), 126.4 (C13), 126.0 (C2), 57.7 (C8), 54.4 (C9, C10), 33.4 (C7), 26.5 (C15), 23.6 (C11, C12), 14.0 (C16).

\section{Z-5a:}

${ }^{1} \mathrm{H}$ NMR (400 MHz, $\mathrm{CDCl}_{3}$ with 0.03\% v/v TMS): $\delta 7.24-7.12\left(\mathrm{~m}, 4 \mathrm{H}, \mathrm{H1}^{\prime}-\mathrm{H} 4{ }^{\prime}\right), 6.50(\mathrm{~d}, J=11.4 \mathrm{~Hz}, 1 \mathrm{H}$, $\left.\mathrm{H}_{13}^{\prime}\right), 5.71\left(\mathrm{dt}, J=11.5,7.4 \mathrm{~Hz}, 1 \mathrm{H}, \mathrm{H} 14^{\prime}\right), 2.86-2.80\left(\mathrm{~m}, 2 \mathrm{H}, \mathrm{H} 7^{\prime}\right), 2.66-2.53\left(\mathrm{~m}, 6 \mathrm{H}, \mathrm{H} 8^{\prime}-\mathrm{H} 10^{\prime}\right), 2.14$ (tdd, $\left.J=7.5,7.4,1.6 \mathrm{~Hz}, 2 \mathrm{H}, \mathrm{H} 15^{\prime}\right), 0.99\left(\mathrm{t}, J=7.5 \mathrm{~Hz}, 8 \mathrm{H}, \mathrm{H} 16^{\prime}\right)$.

${ }^{13} \mathrm{C}\left\{{ }^{1} \mathrm{H}\right\}$ NMR (101 MHz, CDCl 3 with 0.03\% v/v TMS): $\delta 138.7$ (C6'), 136.8 (C5'), 135.2 (C14'), 129.7 (C4'),

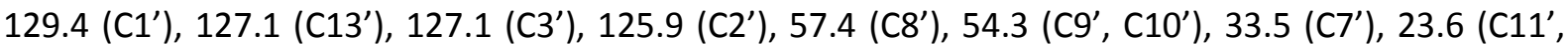
C12'), 21.9 (C15'), 14.5 (C16').

HRMS (ESI) m/z [M+H] calc. for $\mathrm{C}_{16} \mathrm{H}_{24} \mathrm{~N}^{+}$: 230.1903; found: 230.1904 . 
<smiles>c1ccc2cnncc2c1</smiles>

$1 \mathrm{a}$<smiles>CCCC=O</smiles>

$6 a$

$7 \mathbf{a}$
1.) $2.5 \mathrm{~mol} \%$ BDLA THF, $30^{\circ} \mathrm{C}, 15 \mathrm{~h}$ $425-430 \mathrm{~nm}+448 \mathrm{~nm}$

2.) $\mathrm{NaBH}(\mathrm{OAc})_{3}$ $\mathrm{AcOH}$ DCM, rt, $15 \mathrm{~h}$<smiles>CCC=Cc1ccccc1CCN1CCCC1</smiles>

$E-5 a / Z-5 a$

In case the reaction is scaled up it has to be ensured that the employed light source is strong enough or the reaction vessel is designed for photoreactions, i.e. thin diameter/large surface area. Insufficient irradiation let to the formation of IEDDA/amine group transfer product ${ }^{6}$, among others (see $3.00 \mathrm{mmol}-$ Scale below).

\section{$1.50 \mathrm{mmol}-\mathrm{Scale}$ :}

The reaction was set up in a nitrogen filled glovebox.

Phthalazine 1a (394 mg, $3.00 \mathrm{mmol}, 1.00$ equiv) and BDLA (15.4 mg, $75.5 \mu \mathrm{mol}, 2.52 \mathrm{~mol} \%$ ) were suspended in THF (dry, degassed) $(4.00 \mathrm{~mL}$ ). Then, pyrrolidine (7a) (300 $\mu \mathrm{L}, 3.62 \mu \mathrm{mol}, 1.21$ equiv) was added, the reaction vessel was sealed and the remaining part of the reaction was carried out in a fume hood. The colorless solution was heated to $30^{\circ} \mathrm{C}$, and irradiated with two LEDs (425 - $430 \mathrm{~nm}+$ $448 \mathrm{~nm}$ ). Afterwards butyraldehyde (6a) ( $400 \mu \mathrm{L}, 4.35 \mathrm{mmol}, 1.45$ equiv) was added. Gas evolution was observed almost immediately. The reaction was kept irradiated at $30^{\circ} \mathrm{C}$ overnight. Then, the pale yellow solution was concentrated under reduced pressure $\left(10^{-1} \mathrm{mbar}\right.$, volatile compounds were condensed into a cooling trap cooled by liquid nitrogen). The remaining yellow suspension was transferred with DCM (dry, degassed) $(3 \times 4.00 \mathrm{~mL})$ into a Schlenk tube containing $\mathrm{NaBH}(\mathrm{OAc})_{3}$ (670 mg, $1.50 \mathrm{mmol}, 2.00$ equiv). Then, $\mathrm{AcOH}(181 \mu \mathrm{L}, 3.00 \mathrm{mmol}, 2.00$ equiv) was added, and the yellow white suspension was stirred at rt overnight. Afterwards it was quenched by the addition of $1 \mathrm{M}$ $\mathrm{NaOH}$ solution $(30.0 \mathrm{~mL})$. After gas evolution ceased the layers were separated. The aqueous one was extracted with DCM $(3 \times 20.0 \mathrm{~mL})$. The combined organic fractions were washed with brine $(40.0 \mathrm{~mL})$, dried over $\mathrm{Na}_{2} \mathrm{SO}_{4}$, filtered and concentrated under reduced pressure. The crude product was purified by flash column chromatography $\left(\mathrm{SiO}_{2}: 50 \mathrm{~g}\right.$, cyclohexane/EtOAc $\left.+1 \% \mathrm{NEt}_{3}, 2: 1\right)$.

Yield:

mixture of $E / Z$-isomers $E$-5a/Z-5a (1.00: 1.23)

$269 \mathrm{mg}$ (1.17 mmol, 78\%); pale yellow oil. 


\section{$3.00 \mathrm{mmol}-\mathrm{Scale}:$}

Starting materials (solvents were scaled up accordingly):

Phthalazine (1a) (394 mg, $3.00 \mathrm{mmol}, 1.00$ equiv); BDLA (15.4 mg, $75.5 \mu \mathrm{mol}, 2.52 \mathrm{~mol} \%$ ); pyrrolidine (7a) $\left(300 \mu \mathrm{L}, 3.62 \mu \mathrm{mol}, 1.21\right.$ equiv); butyraldehyde (6a) $\left(400 \mu \mathrm{L}, 4.35 \mathrm{mmol}, 1.45\right.$ equiv); $\mathrm{NaBH}(\mathrm{OAc})_{3}$ (1.34 g, $6.00 \mathrm{mmol}, 2.00$ equiv); $\mathrm{AcOH}(361 \mu \mathrm{L}, 6.00 \mathrm{mmol}, 2.00$ equiv).

$\operatorname{LED}(3 \mathrm{x})$ :

$425-430 \mathrm{~nm}+2 \times 448 \mathrm{~nm}$

Temperature:

$30{ }^{\circ} \mathrm{C}$

Purification:

Flash column chromatography $\left(\mathrm{SiO}_{2}: 100 \mathrm{~g}\right.$, cyclohexane/EtOAc $\left.+1 \% \mathrm{NEt}_{3}, 2: 1\right)$.

Yield:

IEDDA/PIRO product

mixture of $E / Z$-isomers $E-5 a / Z-5 a(1.10: 1.00)$

$447 \mathrm{mg}(1.95 \mathrm{mmol}, 65 \%)$; pale yellow oil.

IEDDA/amine group transfer product ${ }^{6}$

$38.3 \mathrm{mg}$ (0.168 mmol, 6\%); pale yellow oil.

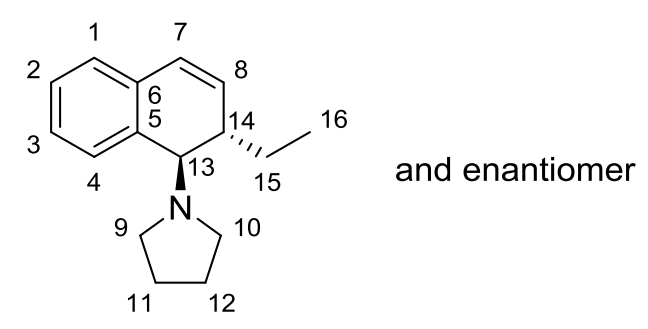

${ }^{1} \mathrm{H} \mathrm{NMR}\left(400 \mathrm{MHz}, \mathrm{CDCl}_{3}\right.$ with $\left.0.03 \% \mathrm{v} / \mathrm{v} \mathrm{TMS}\right): \delta 7.22(\mathrm{td}, J=7.3,1.6 \mathrm{~Hz}, 1 \mathrm{H}, \mathrm{H} 2), 7.16(\mathrm{td}, J=7.3$, $1.5 \mathrm{~Hz}, 1 \mathrm{H}, \mathrm{H} 3$ ), 7.10 (dd, $J=7.4,1.5 \mathrm{~Hz}, 1 \mathrm{H}, \mathrm{H} 4), 7.07$ (dd, $J=7.4,1.4 \mathrm{~Hz}, 1 \mathrm{H}, \mathrm{H} 1$ ), 6.44 (d, $J=9.6 \mathrm{~Hz}$, $1 \mathrm{H}, \mathrm{H7}$ ), 6.07 (ddd, $J=9.6,6.0,1.1 \mathrm{~Hz}, 1 \mathrm{H}, \mathrm{H} 8), 3.47(\mathrm{~s}, 1 \mathrm{H}, \mathrm{H} 13), 2.53-2.39(\mathrm{~m}, 5 \mathrm{H}, \mathrm{H} 9, \mathrm{H} 10, \mathrm{H} 14)$, $1.72-1.59(\mathrm{~m}, 4 \mathrm{H}, \mathrm{H} 11, \mathrm{H} 12), 1.39-1.23(\mathrm{~m}, 2 \mathrm{H}, \mathrm{H} 15), 0.92(\mathrm{t}, J=7.4 \mathrm{~Hz}, 3 \mathrm{H}, \mathrm{H} 16)$. 


\section{Phenethylamine $\mathbf{5 b}$}
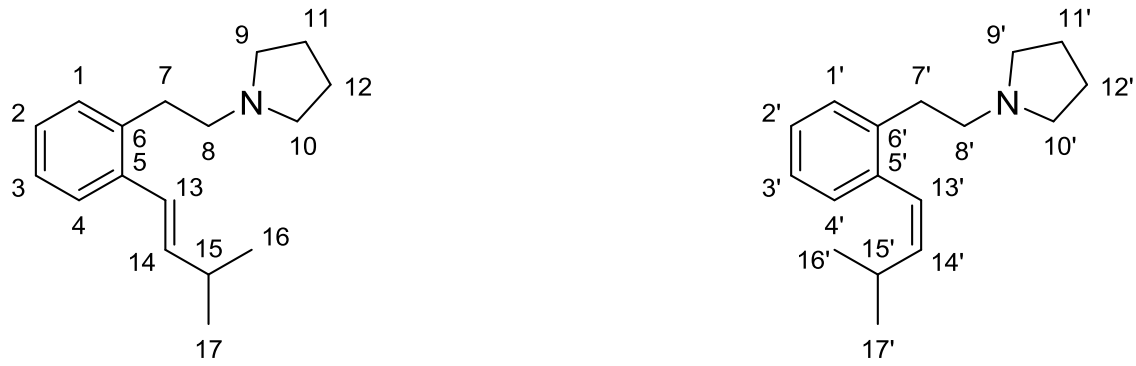

Starting materials:

Phthalazine (1a) $(98.6 \mathrm{mg}, 750 \mu \mathrm{mol}, 1.00$ equiv); BDLA (3.80 mg, $18.6 \mu \mathrm{mol}, 2.49 \mathrm{~mol} \%) ;$ pyrrolidine (7a) $(74.7 \mu \mathrm{L}, 900 \mu \mathrm{mol}, 1.20$ equiv); isovaleraldehyde (6b) $(120 \mu \mathrm{L}, 1.12 \mathrm{mmol}, 1.49$ equiv); $\mathrm{NaBH}(\mathrm{OAc})_{3}$ (328 mg, $1.50 \mathrm{mmol}, 2.00$ equiv); $\mathrm{AcOH}$ (90.4 $\mu \mathrm{L}, 1.50 \mathrm{mmol}, 2.00$ equiv).

LED:

$470 \mathrm{~nm}$

Temperature:

$30{ }^{\circ} \mathrm{C}$

Purification:

Flash column chromatography $\left(\mathrm{SiO}_{2}: 20 \mathrm{~g}\right.$, cyclohexane/EtOAc + 1\% $\left.\mathrm{NEt}_{3}, 5: 1\right)$.

Yield: mixture of $E / Z$-isomers $E-5 b / Z-5 b(1.16: 1.00)$

$132 \mathrm{mg}(543 \mu \mathrm{mol}, 72 \%)$; pale yellow oil.

\section{E-5b:}

${ }^{1} \mathrm{H} \mathrm{NMR}\left(400 \mathrm{MHz}, \mathrm{CDCl}_{3}\right.$ with $\left.0.03 \% \mathrm{v} / \mathrm{v} \mathrm{TMS}\right): \delta 7.45-7.39(\mathrm{~m}, \mathrm{H} 4), 7.23-7.12(\mathrm{~m}, 3 \mathrm{H}, \mathrm{H} 1, \mathrm{H} 2, \mathrm{H} 3)$, $6.62(\mathrm{dd}, J=15.7,0.9 \mathrm{~Hz}, 1 \mathrm{H}, \mathrm{H} 13), 6.06$ (dd, $J=15.7,7.0 \mathrm{~Hz}, 1 \mathrm{H}, \mathrm{H} 14), 2.95-2.88(\mathrm{~m}, 2 \mathrm{H}, \mathrm{H7}), 2.67-$ $2.55(\mathrm{~m}, 6 \mathrm{H}, \mathrm{H} 8-\mathrm{H} 10), 2.55-2.44(\mathrm{~m}, 1 \mathrm{H}, \mathrm{H} 15), 1.88-1.77(\mathrm{~m}, 7 \mathrm{H}, \mathrm{H} 11, \mathrm{H} 12), 1.10(\mathrm{~d}, \mathrm{~J}=6.7 \mathrm{~Hz}, 4 \mathrm{H}$, H16, H17).

${ }^{13} \mathrm{C}\left\{{ }^{1} \mathrm{H}\right\}$ NMR (101 MHz, $\mathrm{CDCl}$ with $0.03 \% \mathrm{v} / \mathrm{v}$ TMS): $\delta 140.1(14), 138.51\left(\mathrm{C}_{\mathrm{Ar}}\right.$ or $\left.\mathrm{C}_{\mathrm{Ar}}{ }^{\prime}\right), 137.27\left(\mathrm{C}_{\mathrm{Ar}}\right.$ or $\left.\mathrm{C}_{\mathrm{Ar}}{ }^{\prime}\right)$, $137.11\left(\mathrm{C}_{\mathrm{Ar}}\right.$ or $\left.\mathrm{C}_{\mathrm{Ar}}{ }^{\prime}\right), 137.01\left(\mathrm{C}_{\mathrm{Ar}}\right.$ or $\left.\mathrm{C}_{\mathrm{Ar}}{ }^{\prime}\right), 129.87\left(\mathrm{C}_{\mathrm{Ar}}\right.$ or $\left.\mathrm{C}_{\mathrm{Ar}}{ }^{\prime}\right), 129.60\left(\mathrm{C}_{\mathrm{Ar}}\right.$ or $\left.\mathrm{C}_{\mathrm{Ar}}{ }^{\prime}\right), 129.41\left(\mathrm{C}_{\mathrm{Ar}}\right.$ or $\left.\mathrm{C}_{\mathrm{Ar}}{ }^{\prime}\right)$, $127.08\left(\mathrm{C}_{\mathrm{Ar}}\right.$ or $\left.\mathrm{C}_{\mathrm{Ar}}{ }^{\prime}\right), 127.06\left(\mathrm{C}_{\mathrm{Ar}}\right.$ or $\left.\mathrm{C}_{\mathrm{Ar}}{ }^{\prime}\right), 126.60\left(\mathrm{C}_{\mathrm{Ar}}\right.$ or $\left.\mathrm{C}_{\mathrm{Ar}}{ }^{\prime}\right), 126.04(\mathrm{C} 4), 125.95\left(\mathrm{C}_{\mathrm{Ar}}\right.$ or $\left.\mathrm{C}_{\mathrm{Ar}}{ }^{\prime}\right), 124.4(\mathrm{C} 13)$, 57.6 (C8), 54.4 (C9, C10), 33.3 (C7), 31.9 (C15), 23.6 (C11, C12), 22.7 (C16, C17).

\section{Z-5b:}

${ }^{1} \mathrm{H}$ NMR (400 MHz, $\mathrm{CDCl}_{3}$ with $\left.0.03 \% \mathrm{v} / \mathrm{v} \mathrm{TMS}\right): \delta 7.23-7.12\left(\mathrm{~m}, 4 \mathrm{H}, \mathrm{H}^{\prime}-\mathrm{H} 4^{\prime}\right), 6.41(\mathrm{~d}, \mathrm{~J}=11.4 \mathrm{~Hz}, 1 \mathrm{H}$, $\left.\mathrm{H} 13^{\prime}\right), 5.53\left(\mathrm{dd}, J=11.3,10.3 \mathrm{~Hz}, 1 \mathrm{H}, \mathrm{H} 14^{\prime}\right), 2.87-2.80\left(\mathrm{~m}, 2 \mathrm{H}, \mathrm{H} 7^{\prime}\right), 2.67-2.55\left(\mathrm{~m}, 7 \mathrm{H}, \mathrm{H} 8^{\prime}-\mathrm{H} 10^{\prime}\right.$, $\left.H 15^{\prime}\right), 1.88-1.77\left(m, 7 H, H 11^{\prime}, H 12^{\prime}\right), 0.98$ (d, J = 6.6 Hz, 4H, H16', H17').

${ }^{13} \mathrm{C}\left\{{ }^{1} \mathrm{H}\right\}$ NMR (101 MHz, $\mathrm{CDCl}_{3}$ with 0.03\% v/v TMS): $\delta 140.9$ (C14'), 138.51 ( $\mathrm{C}_{\mathrm{Ar}}$ or $\left.\mathrm{C}_{\mathrm{Ar}}{ }^{\prime}\right), 137.27\left(\mathrm{C}_{\mathrm{Ar}}\right.$ or $\left.\mathrm{C}_{\mathrm{Ar}}{ }^{\prime}\right), 137.11\left(\mathrm{C}_{\mathrm{Ar}}\right.$ or $\left.\mathrm{C}_{\mathrm{Ar}}{ }^{\prime}\right), 137.01\left(\mathrm{C}_{\mathrm{Ar}}\right.$ or $\left.\mathrm{C}_{\mathrm{Ar}}{ }^{\prime}\right), 129.87\left(\mathrm{C}_{\mathrm{Ar}}\right.$ or $\left.\mathrm{C}_{\mathrm{Ar}}{ }^{\prime}\right), 129.60\left(\mathrm{C}_{\mathrm{Ar}}\right.$ or $\left.\mathrm{C}_{\mathrm{Ar}}{ }^{\prime}\right), 129.41\left(\mathrm{C}_{\mathrm{Ar}}\right.$ or $\left.\mathrm{C}_{\mathrm{Ar}}{ }^{\prime}\right)$, $127.08\left(C_{\mathrm{Ar}}\right.$ or $\left.\mathrm{C}_{\mathrm{Ar}}{ }^{\prime}\right), 127.06\left(\mathrm{C}_{\mathrm{Ar}}\right.$ or $\left.\mathrm{C}_{\mathrm{Ar}}{ }^{\prime}\right), 126.60\left(\mathrm{C}_{\mathrm{Ar}}\right.$ or $\left.\mathrm{C}_{\mathrm{Ar}}{ }^{\prime}\right), 126.04\left(\mathrm{C}_{\mathrm{Ar}}\right.$ or $\left.\mathrm{C}_{\mathrm{Ar}}{ }^{\prime}\right), 125.95(\mathrm{C} 4), 125.4\left(\mathrm{C} 13^{\prime}\right)$, $57.4\left(\mathrm{C}^{\prime}\right), 54.3$ (C9', C10'), $33.4\left(\mathrm{C}^{\prime}\right), 27.3$ (C15'), 23.6 (C11', C12'), 23.25 (C16', C17').

HRMS (ESI) m/z [M+H] calc. for $\mathrm{C}_{17} \mathrm{H}_{26} \mathrm{~N}^{+}$: 244.2060; found: 244.2057 . 


\section{Phenethylamine 5c}
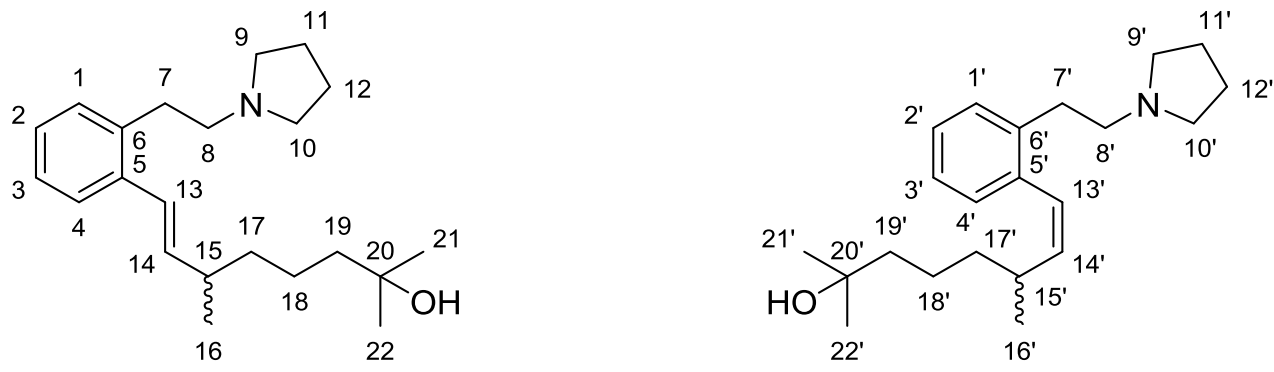

Starting materials:

Phthalazine (1a) $(98.6 \mathrm{mg}, 750 \mu \mathrm{mol}, 1.00$ equiv); BDLA (3.80 mg, $18.6 \mu \mathrm{mol}, 2.49 \mathrm{~mol} \%)$; pyrrolidine (7a) $(74.7 \mu \mathrm{L}, 900 \mu \mathrm{mol}, 1.20$ equiv); 3,7-dimethyl-7-hydroxyoctanal (6c) (200 $\mu \mathrm{L}, 1.06 \mathrm{mmol}$, 1.41 equiv); $\mathrm{NaBH}(\mathrm{OAc})_{3}$ (328 mg, $1.50 \mathrm{mmol}, 2.00$ equiv); $\mathrm{AcOH}(90.4 \mu \mathrm{L}, 1.50 \mathrm{mmol}, 2.00$ equiv).

LED:

$470 \mathrm{~nm}$

Temperature:

$50{ }^{\circ} \mathrm{C}$

Purification:

Flash column chromatography $\left(\mathrm{SiO}_{2}: 20 \mathrm{~g}\right.$, cyclohexane/EtOAc $+1 \% \mathrm{NEt}_{3}, 2: 1$ to 1:1).

Yield: mixture of $E / Z$-isomers $E-5 c / Z-5 c(1.46: 1.00)$

$175 \mathrm{mg}(531 \mu \mathrm{mol}, 71 \%)$; pale yellow oil.

$E-5 c$ :

${ }^{1} \mathrm{H} \mathrm{NMR}\left(400 \mathrm{MHz}, \mathrm{CDCl}_{3}\right.$ with $\left.0.03 \% \mathrm{v} / \mathrm{v} \mathrm{TMS}\right): \delta 7.44-7.36(\mathrm{~m}, 1 \mathrm{H}, \mathrm{H} 4), 7.21-7.11(\mathrm{~m}, 3 \mathrm{H}, 1,2,3)$, $6.62(\mathrm{~d}, J=15.6 \mathrm{~Hz}, 1 \mathrm{H}, \mathrm{H} 13), 5.91(\mathrm{dd}, J=15.6,8.0 \mathrm{~Hz}, 1 \mathrm{H}, \mathrm{H} 14), 2.94-2.86(\mathrm{~m}, 2 \mathrm{H}, \mathrm{H} 7), 2.67-2.54$ $(\mathrm{m}, 6 \mathrm{H}, \mathrm{H} 8, \mathrm{H} 9, \mathrm{H} 10), 2.34(\mathrm{dt}, J=13.2,6.7 \mathrm{~Hz}, 1 \mathrm{H}, \mathrm{H} 15), 1.99(\mathrm{~s}, \mathrm{br},-\mathrm{OH}), 1.88-1.76(\mathrm{~m}, 4 \mathrm{H}, 11,12)$, $1.57-1.21(\mathrm{~m}, 6 \mathrm{H}, 17,18,19), 1.14(\mathrm{~s}, 4 \mathrm{H}, \mathrm{H} 21, \mathrm{H} 22), 1.09$ (d, J = 6.7 Hz, 3H, H16).

${ }^{13} \mathrm{C}\left\{{ }^{1} \mathrm{H}\right\}$ NMR (101 MHz, $\mathrm{CDCl}_{3}$ with $0.03 \% \mathrm{v} / \mathrm{v} \mathrm{TMS}$ ): $\delta 138.8$ (C14), 137.3 (C6), 137.2 (C5), 129.8 (C1 or C2 or C3), 127.1 (C1 or C2 or C3), 126.6 (C1 or C2 or C3), 126.3 (C4), 126.1 (C13), 125.89, 70.8 (C20), 57.8 (C8), 54.3 (C9, C10), 44.1 (C19), 37.6 (C17), 37.5 (C15), 33.3 (C7), 29.4 (C21, C22), 23.5 (C11, C12), 22.1 (C18), 21.1 (C16).

Z-5c:

${ }^{1} \mathrm{H} \mathrm{NMR}\left(400 \mathrm{MHz}, \mathrm{CDCl}_{3}\right.$ with $\left.0.03 \% \mathrm{v} / \mathrm{v} \mathrm{TMS}\right): \delta 7.21-7.11\left(\mathrm{~m}, 4 \mathrm{H}, 1^{\prime}, 2^{\prime}, 3^{\prime}, 4^{\prime}\right), 6.47(\mathrm{~d}, J=11.5 \mathrm{~Hz}$, $\left.1 \mathrm{H}, \mathrm{H} 13^{\prime}\right), 5.49$ (dd, $\left.J=11.4,10.5 \mathrm{~Hz}, 1 \mathrm{H}, \mathrm{H} 14^{\prime}\right), 2.87-2.80\left(\mathrm{~m}, 2 \mathrm{H}, \mathrm{H} 7^{\prime}\right), 2.67-2.54\left(\mathrm{~m}, 6 \mathrm{H}, 8^{\prime}, 9^{\prime}, 10^{\prime}\right)$, $2.48\left(\mathrm{dq}, J=12.8,6.4 \mathrm{~Hz}, 1 \mathrm{H}, \mathrm{H} 15^{\prime}\right), 1.99(\mathrm{~s}, \mathrm{br},-\mathrm{OH}), 1.88-1.76\left(\mathrm{~m}, 4 \mathrm{H}, 11^{\prime}, 12^{\prime}\right), 1.57-1.21(\mathrm{~m}, 6 \mathrm{H}$, $\left.17^{\prime}, 18^{\prime}, 19^{\prime}\right), 1.20\left(\mathrm{~s}, 6 \mathrm{H}, 21^{\prime}, 22^{\prime}\right), 1.00\left(\mathrm{~d}, J=6.6 \mathrm{~Hz}, 2 \mathrm{H}, 16^{\prime}\right)$.

${ }^{13} \mathrm{C}\left\{{ }^{1} \mathrm{H}\right\}$ NMR (101 MHz, CDCl 3 with 0.03\% v/v TMS): $\delta 139.7$ (C14'), 138.5 (C6'), 137.0 (C5'), 129.5 (C1' or $\mathrm{C2}^{\prime}$ or $\mathrm{C} 3^{\prime}$ or $\left.\mathrm{C} 4^{\prime}\right), 129.2\left(\mathrm{C1}^{\prime}\right.$ or $\mathrm{C} 2^{\prime}$ or $\mathrm{C} 3^{\prime}$ or $\left.\mathrm{C} 4^{\prime}\right), 127.1$ (C1 $^{\prime}$ or $\mathrm{C} 2^{\prime}$ or $\mathrm{C} 3^{\prime}$ or $\left.\mathrm{C} 4^{\prime}\right), 126.5\left(\mathrm{C} 13^{\prime}\right), 125.9$

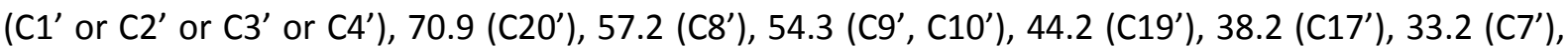
$32.3\left(\mathrm{C} 15^{\prime}\right), 29.5$ (C21', C22'), 23.5 (C11', C12'), 22.3 (C18'), 21.3 (C16').

HRMS (ESI) m/z [M+H] $]^{+}$calc. for $\mathrm{C}_{22} \mathrm{H}_{36} \mathrm{NO}^{+}: 330.2791$; found: 330.2790 . 


\section{Phenethylamine 5d}
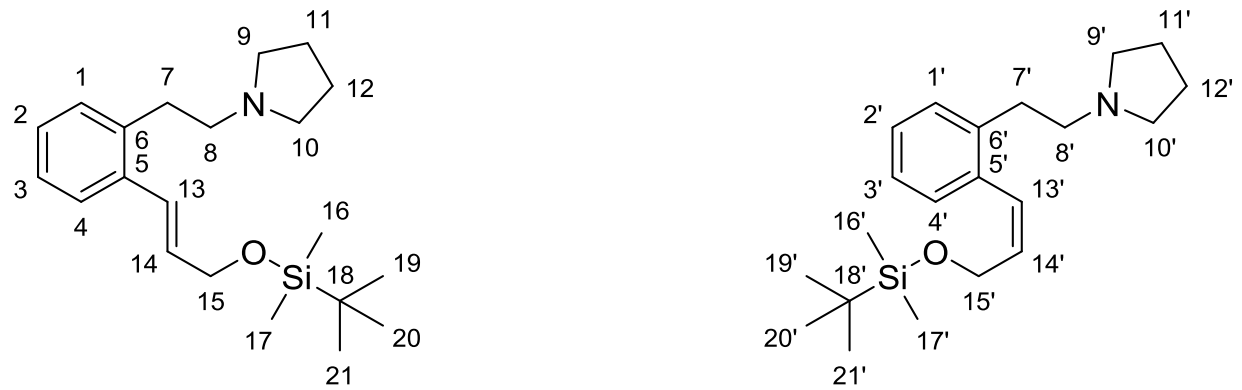

Starting materials:

Phthalazine (1a) $(98.6 \mathrm{mg}, 750 \mu \mathrm{mol}, 1.00$ equiv); BDLA (3.80 mg, $18.6 \mu \mathrm{mol}, 2.49 \mathrm{~mol} \%) ;$ pyrrolidine (7a) $\left(74.7 \mu \mathrm{L}, 900 \mu \mathrm{mol}, 1.20\right.$ equiv); aldehyde $6 \mathrm{~d}\left(207 \mathrm{mg}, 1.09 \mathrm{mmol}, 1.45\right.$ equiv); $\mathrm{NaBH}(\mathrm{OAc})_{3}$ (328 mg, $1.50 \mathrm{mmol}, 2.00$ equiv); $\mathrm{AcOH}(90.4 \mu \mathrm{L}, 1.50 \mathrm{mmol}, 2.00$ equiv).

LED:

$425-430 \mathrm{~nm}$

Temperature:

$30{ }^{\circ} \mathrm{C}$

Purification:

Flash column chromatography (2 times) $\left(\mathrm{SiO}_{2}: 20 \mathrm{~g}\right.$, cyclohexane/EtOAc $\left.+3 \% \mathrm{NEt}_{3}, 10: 1\right)$.

Yield: mixture of $E / Z$-isomers $E-5 \mathrm{~d} / \mathbf{Z}-\mathbf{5 d}(1.00: 1.53)$

$129 \mathrm{mg}(374 \mu \mathrm{mol}, 50 \%) ;$ pale yellow oil.

\section{E-5d:}

${ }^{1} \mathrm{H}$ NMR (400 MHz, $\mathrm{CDCl}_{3}$ with 0.03\% v/v TMS): $\delta 7.49-7.41(\mathrm{~m}, 1 \mathrm{H}, \mathrm{H} 4), 7.23-7.13(\mathrm{~m}, 3 \mathrm{H}, \mathrm{H} 1, \mathrm{H} 2$, H3), 6.88 (dt, $J=15.7,1.9 \mathrm{~Hz}, 1 \mathrm{H}, \mathrm{H} 13), 6.19$ (dt, $J=15.6,5.1 \mathrm{~Hz}, 1 \mathrm{H}, \mathrm{H} 14), 4.37$ (dd, $J=5.0,1.8 \mathrm{~Hz}, 2 \mathrm{H}$, H15), $2.95-2.87(m, 2 H, H 7), 2.67-2.53(m, 6 H, H 8-H 10), 1.86-1.75(m, 4 H, H 11, H 12), 0.94(s, 9 H$, $\mathrm{H} 19-\mathrm{H} 21), 0.12(\mathrm{~s}, 6 \mathrm{H}, \mathrm{H} 16, \mathrm{H} 17)$.

${ }^{13} \mathrm{C}\left\{{ }^{1} \mathrm{H}\right\}$ NMR (101 MHz, CDCl with 0.03\% v/v TMS): $\delta 137.9$ (C6), 136.1 (C5), 131.1 (C14), 130.0 (C1), 127.6 (C2 or C3), 126.9 (C13), 126.6 (C2 or C3), 126.2 (C4), 64.2 (15) 57.8 (C8), 54.3 (H9, H10), 33.3 (C7), 26.1 (C19 - C21), 23.6 (C11, C12), 18.6 (C18), -4.95 (C16, C17).

\section{Z-5d:}

${ }^{1} \mathrm{H}$ NMR (400 MHz, $\mathrm{CDCl}_{3}$ with 0.03\% v/v TMS): $\delta 7.23-7.13\left(\mathrm{~m}, 3 \mathrm{H}, \mathrm{H}^{\prime}, \mathrm{H}^{\prime}, \mathrm{H} 3^{\prime}\right), 7.07$ (d, $J=7.1 \mathrm{~Hz}$, $1 \mathrm{H}, \mathrm{H} 4^{\prime}$ ), $6.64\left(\mathrm{dt}, J=11.5,1.7 \mathrm{~Hz}, 1 \mathrm{H}, \mathrm{H} 13^{\prime}\right), 5.87\left(\mathrm{dt}, J=11.6,6.3 \mathrm{~Hz}, 1 \mathrm{H}, \mathrm{H} 14^{\prime}\right), 4.29$ (dd, $J=6.3,1.6 \mathrm{~Hz}$, $\left.2 \mathrm{H}, \mathrm{H} 15^{\prime}\right), 2.87-2.81\left(\mathrm{~m}, 2 \mathrm{H}, \mathrm{H} 7^{\prime}\right), 2.67-2.53\left(\mathrm{~m}, 6 \mathrm{H}, \mathrm{H} 8^{\prime}-\mathrm{H} 10^{\prime}\right), 1.86-1.75\left(\mathrm{~m}, 4 \mathrm{H}, \mathrm{H} 11^{\prime}, \mathrm{H} 12^{\prime}\right)$, $0.87\left(\mathrm{~s}, 9 \mathrm{H}, \mathrm{H} 19^{\prime}-\mathrm{H} 21^{\prime}\right), 0.01\left(\mathrm{~s}, 6 \mathrm{H}, \mathrm{H} 16^{\prime}, \mathrm{H} 17^{\prime}\right)$.

${ }^{13} \mathrm{C}\left\{{ }^{1} \mathrm{H}\right\}$ NMR (101 MHz, $\mathrm{CDCl}_{3}$ with $0.03 \%$ v/v TMS): $\delta 138.7$ (C6'), 135.7 (C5'), 132.8 (C14'), 129.6 (C1'), $129.6\left(\mathrm{C}^{\prime}\right), 128.5\left({\left.\mathrm{C} 13^{\prime}\right)}^{\prime}\right), 127.6\left(\mathrm{C} 2^{\prime}\right), 125.9\left(\mathrm{C} 3^{\prime}\right), 60.4\left(\mathrm{C} 15^{\prime}\right), 57.4\left(\mathrm{C} 8^{\prime}\right), 54.3\left(\mathrm{C} 9^{\prime}, \mathrm{C} 10^{\prime}\right), 33.5$ (C7'), 26.1 (C19'-C21'), 23.6 (C11', C12'), 18.4 (C18'), -4.98 (C16', C17').

HRMS (ESI) m/z [M+H] $]^{+}$calc. for $\mathrm{C}_{21} \mathrm{H}_{36} \mathrm{NOSi}^{+}$: 346.2561; found: 346.2565 . 


\section{Phenethylamine 5e}
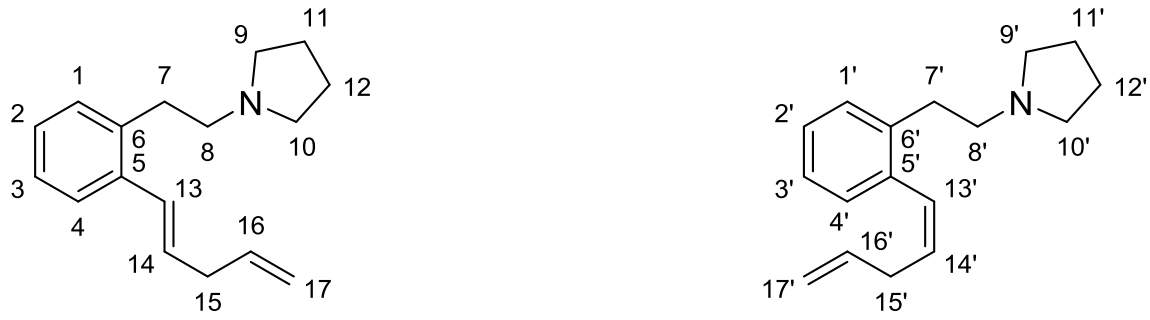

Starting materials:

Phthalazine (1a) $(98.6 \mathrm{mg}, 750 \mu \mathrm{mol}, 1.00$ equiv); BDLA (3.80 mg, $18.6 \mu \mathrm{mol}, 2.49 \mathrm{~mol} \%) ;$ pyrrolidine (7a) $\left(74.7 \mu \mathrm{L}, 900 \mu \mathrm{mol}, 1.20\right.$ equiv); pent-4-enal (6e) $\left(100 \mu \mathrm{L}, 982 \mu \mathrm{mol}, 1.31\right.$ equiv); $\mathrm{NaBH}(\mathrm{OAc})_{3}$ (328 mg, $1.50 \mathrm{mmol}, 2.00$ equiv); $\mathrm{AcOH}$ (90.4 $\mu \mathrm{L}, 1.50 \mathrm{mmol}, 2.00$ equiv).

LED:

$448 \mathrm{~nm}$

Temperature:

$30{ }^{\circ} \mathrm{C}$

Purification:

Flash column chromatography (2 times) $\left(\mathrm{SiO}_{2}: 20 \mathrm{~g}\right.$, cyclohexane/EtOAc $+1 \% \mathrm{NEt}_{3}, 10: 1$ to $\left.5: 1\right)$.

Yield: mixture of E/Z-isomers E-5e/Z-5e (1.24 : 1.00)

$138 \mathrm{mg}$ (573 $\mu \mathrm{mol}, 76 \%)$.

\section{$E-5 e:$}

${ }^{1} \mathrm{H}$ NMR (400 MHz, $\mathrm{CDCl}_{3}$ with 0.03\% v/v TMS): $\delta 7.46-7.40(\mathrm{~m}, 1 \mathrm{H}, \mathrm{H} 4), 7.24-7.13(\mathrm{~m}, 3 \mathrm{H}, \mathrm{H} 1, \mathrm{H} 2$, H3), $6.69(\mathrm{dt}, J=15.6,1.7 \mathrm{~Hz}, 1 \mathrm{H}, \mathrm{H} 13), 6.11(\mathrm{dt}, J=15.6,6.7 \mathrm{~Hz}, 1 \mathrm{H}, \mathrm{H} 14), 5.97-5.80(\mathrm{~m}, 1 \mathrm{H}, 16), 5.15$ - $4.99(\mathrm{~m}, 2 \mathrm{H}, \mathrm{H} 17), 3.01-2.95(\mathrm{~m}, 2 \mathrm{H}, \mathrm{H} 15), 2.94-2.79(\mathrm{~m}, 2 \mathrm{H}, \mathrm{H} 7), 2.67-2.52(\mathrm{~m}, 6 \mathrm{H}, \mathrm{H} 8, \mathrm{H} 9, \mathrm{H} 10)$, $1.87-1.76(\mathrm{~m}, 4 \mathrm{H}, \mathrm{H} 11, \mathrm{H} 12)$.

${ }^{13} \mathrm{C}\left\{{ }^{1} \mathrm{H}\right\}$ NMR (101 MHz, CDCl 3 with 0.03\% v/v TMS): $\delta 137.4$ (C6), 136.7 (C16), 136.3 (C5), 130.21 ( $\mathrm{C}_{\mathrm{Ar}}$ or $\left.C_{\mathrm{Ar}}{ }^{\prime}\right), 129.9(\mathrm{C} 14), 129.52$ ( $\mathrm{C}_{\mathrm{Ar}}$ or $\left.\mathrm{C}_{\mathrm{Ar}}{ }^{\prime}\right), 129.46\left(\mathrm{C}_{\mathrm{Ar}}\right.$ or $\left.\mathrm{C}_{\mathrm{Ar}}{ }^{\prime}\right), 128.6(\mathrm{C} 13), 127.33\left(\mathrm{C}_{\mathrm{Ar}}\right.$ or $\left.\mathrm{C}_{\mathrm{Ar}}{ }^{\prime}\right), 127.32\left(\mathrm{C}_{\mathrm{Ar}}\right.$ or $\left.\mathrm{C}_{\mathrm{Ar}}{ }^{\prime}\right), 126.6\left(\mathrm{C}_{\mathrm{Ar}}\right.$ or $\left.\mathrm{C}_{\mathrm{Ar}}{ }^{\prime}\right), 126.1(\mathrm{C} 4), 126.0$ ( $\mathrm{C}_{\mathrm{Ar}}$ or $\left.\mathrm{C}_{\mathrm{Ar}}{ }^{\prime}\right), 115.8$ (C17), 57.7 (C8), 54.4 (C9, C10), 37.4 (C15), 33.3 (C7), 23.6 (C11, C12).

Z-5e:

${ }^{1} \mathrm{H}$ NMR (400 MHz, $\mathrm{CDCl}_{3}$ with $\left.0.03 \% \mathrm{v} / \mathrm{v} \mathrm{TMS}\right): \delta 7.24-7.13\left(\mathrm{~m}, 4 \mathrm{H}, \mathrm{H}_{\mathrm{Ar}}{ }^{\prime}\right), 6.64(\mathrm{dt}, J=11.4,1.6 \mathrm{~Hz}, 1 \mathrm{H}$, $\left.\mathrm{H} 13^{\prime}\right), 5.97-5.80\left(\mathrm{~m}, 1 \mathrm{H}, \mathrm{H} 16^{\prime}\right), 5.77\left(\mathrm{dt}, J=11.4,7.6 \mathrm{~Hz}, 1 \mathrm{H}, \mathrm{H} 14^{\prime}\right), 5.15-4.99\left(\mathrm{~m}, 2 \mathrm{H}, \mathrm{H} 17^{\prime}\right), 2.94-$ $2.79\left(\mathrm{~m}, 4 \mathrm{H}, \mathrm{H} 7^{\prime}, \mathrm{H} 15^{\prime}\right), 2.67-2.52\left(\mathrm{~m}, 6 \mathrm{H}, \mathrm{H} 8^{\prime}, \mathrm{H} 9^{\prime}, \mathrm{H} 10^{\prime}\right), 1.87-1.76\left(\mathrm{~m}, 4 \mathrm{H}, \mathrm{H} 11^{\prime}, \mathrm{H} 12^{\prime}\right)$.

${ }^{13} \mathrm{C}\left\{{ }^{1} \mathrm{H}\right\}$ NMR (101 MHz, $\mathrm{CDCl}{ }_{3}$ with 0.03\% v/v TMS): $\delta 138.7\left(\mathrm{C}^{\prime}\right), 136.9\left(\mathrm{C} 16^{\prime}\right), 130.2\left(\mathrm{C}_{\mathrm{Ar}}\right.$ or $\left.\mathrm{C}_{\mathrm{Ar}}{ }^{\prime}\right), 130.1$ $\left(\mathrm{C} 14^{\prime}\right), 129.52\left(\mathrm{C}_{\mathrm{Ar}}\right.$ or $\left.\mathrm{C}_{\mathrm{Ar}}{ }^{\prime}\right), 129.46\left(\mathrm{C}_{\mathrm{Ar}}\right.$ or $\left.\mathrm{C}_{\mathrm{Ar}}{ }^{\prime}\right), 129.0\left(\mathrm{C}^{\prime} 3^{\prime}\right), 127.33\left(\mathrm{C}_{\mathrm{Ar}}\right.$ or $\left.\mathrm{C}_{\mathrm{Ar}}{ }^{\prime}\right), 127.32\left(\mathrm{C}_{\mathrm{Ar}}\right.$ or $\left.\mathrm{C}_{\mathrm{Ar}}{ }^{\prime}\right)$, $126.6\left(\mathrm{C}_{\mathrm{Ar}}\right.$ or $\left.\mathrm{C}_{\mathrm{Ar}}{ }^{\prime}\right), 126.1\left(\mathrm{C}_{\mathrm{Ar}}\right.$ or $\left.\mathrm{C}_{\mathrm{Ar}}{ }^{\prime}\right), 126.0\left(\mathrm{C}_{\mathrm{Ar}}\right.$ or $\left.\mathrm{C}_{\mathrm{Ar}}{ }^{\prime}\right), 115.2\left(\mathrm{C} 17^{\prime}\right), 57.4\left(\mathrm{C} 8^{\prime}\right), 54.3\left(\mathrm{C}{ }^{\prime}, \mathrm{C} 10^{\prime}\right), 33.5$ $\left(C 7^{\prime}\right), 32.7\left(\mathrm{C} 15^{\prime}\right), 23.6\left(\mathrm{C} 11^{\prime}, \mathrm{C} 12^{\prime}\right)$.

HRMS (ESI) m/z [M+H] calc. for $\mathrm{C}_{17} \mathrm{H}_{24} \mathrm{~N}^{+}$: 242.1903; found: 242.1902 . 


\section{Phenethylamine $\mathbf{5 f}$}<smiles>CC=Cc1ccccc1CCN1CCOCC1</smiles><smiles></smiles>

Starting materials:

Phthalazine (1a) (98.6 mg, $750 \mu \mathrm{mol}, 1.00$ equiv); BDLA (3.80 mg, $18.6 \mu \mathrm{mol}, 2.49 \mathrm{~mol} \%$ ); morpholine (7b) $\left(79.6 \mu \mathrm{L}, 900 \mu \mathrm{mol}, 1.20\right.$ equiv); butyraldehyde (6a) $\left(100 \mu \mathrm{L}, 1.09 \mathrm{mmol}, 1.45\right.$ equiv); $\mathrm{NaBH}(\mathrm{OAc})_{3}$ (328 mg, $1.50 \mathrm{mmol}, 2.00$ equiv); $\mathrm{AcOH}(90.4 \mu \mathrm{L}, 1.50 \mathrm{mmol}, 2.00$ equiv).

LED:

$470 \mathrm{~nm}$

Temperature:

$45^{\circ} \mathrm{C}$

Purification:

Flash column chromatography (2 times) $\left(\mathrm{SiO}_{2}: 20 \mathrm{~g}\right.$, cyclohexane/EtOAc $\left.+1 \% \mathrm{NEt}_{3}, 2: 1\right)$.

Yield: mixture of $E / Z$-isomers $E-5 f / Z-5 f(1.00: 1.12)$

$149 \mathrm{mg}$, (606 mmol, 81\%); pale yellow oil.

\section{$E-5 f:$}

${ }^{1} \mathrm{H}$ NMR $\left(400 \mathrm{MHz}, \mathrm{CDCl}_{3}\right.$ with $\left.0.03 \% \mathrm{v} / \mathrm{v} \mathrm{TMS}\right): \delta 7.44-7.40(\mathrm{~m}, 1 \mathrm{H}, \mathrm{H} 4), 7.22-7.12(\mathrm{~m}, 3 \mathrm{H}, \mathrm{H} 1, \mathrm{H} 2$, $\mathrm{H} 3), 6.62(\mathrm{dt}, J=15.5,1.7 \mathrm{~Hz}, 1 \mathrm{H}, \mathrm{H} 13), 6.15(\mathrm{dt}, J=15.6,6.6 \mathrm{~Hz}, 1 \mathrm{H}, \mathrm{H} 14), 3.79-3.72(\mathrm{~m}, 4 \mathrm{H}, \mathrm{H} 11$, $\mathrm{H} 12), 2.90-2.84(\mathrm{~m}, 2 \mathrm{H}, \mathrm{H} 7), 2.57-2.47(\mathrm{~m}, 6 \mathrm{H}, \mathrm{H} 8, \mathrm{H} 9, \mathrm{H} 10), 2.32-2.21(\mathrm{~m}, 2 \mathrm{H}, \mathrm{H} 15), 1.10$ (t, J = $7.5 \mathrm{~Hz}, 3 \mathrm{H}, \mathrm{H} 16)$.

${ }^{13} \mathrm{C}\left\{{ }^{1} \mathrm{H}\right\}$ NMR (101 MHz, $\mathrm{CDCl}_{3}$ with 0.03\% v/v TMS): $\delta 136.9$ (C5), 136.8 (C6), 134.8 (C14), 129.9 (C1), $129.7\left(\mathrm{C}_{\mathrm{Ar}}\right.$ or $\left.\mathrm{C}_{\mathrm{Ar}}{ }^{\prime}\right), 127.13\left(\mathrm{C}_{\mathrm{Ar}}\right.$ or $\left.\mathrm{C}_{\mathrm{Ar}}{ }^{\prime}\right), 127.10$ ( $\mathrm{C}_{\mathrm{Ar}}$ or $\left.\mathrm{C}_{\mathrm{Ar}}{ }^{\prime}\right), 126.7\left(\mathrm{C}_{\mathrm{Ar}}\right.$ or $\left.\mathrm{C}_{\mathrm{Ar}}{ }^{\prime}\right), 126.3(\mathrm{C} 13), 126.1(\mathrm{C} 4)$, $126.0\left(\mathrm{C}_{\mathrm{Ar}}\right.$ or $\left.\mathrm{C}_{\mathrm{Ar}}{ }^{\prime}\right), 67.1$ (C11, $\left.\mathrm{C} 12\right), 60.2$ (C8), 53.8 (C9, $\left.\mathrm{C} 10\right), 30.7$ (C7), 26.5 (C15), 13.9 (C16).

\section{Z-5f:}

${ }^{1} \mathrm{H}$ NMR (400 MHz, $\mathrm{CDCl}_{3}$ with $\left.0.03 \% \mathrm{v} / \mathrm{v} \mathrm{TMS}\right): \delta 7.22-7.12\left(\mathrm{~m}, 4 \mathrm{H}, \mathrm{H}_{\mathrm{Ar}}{ }^{\prime}\right), 6.48(\mathrm{dt}, J=11.4,1.7 \mathrm{~Hz}, 1 \mathrm{H}$, $\left.\mathrm{H}_{13}^{\prime}\right), 5.73\left(\mathrm{dt}, J=11.4,7.4 \mathrm{~Hz}, 1 \mathrm{H}, \mathrm{H} 14^{\prime}\right), 3.79-3.72\left(\mathrm{~m}, 4 \mathrm{H}, \mathrm{H} 11^{\prime}, \mathrm{H} 12^{\prime}\right), 2.82-2.77\left(\mathrm{~m}, 2 \mathrm{H}, \mathrm{H} 7^{\prime}\right), 2.57$ $-2.47\left(\mathrm{~m}, 6 \mathrm{H}, \mathrm{H} 8^{\prime}, \mathrm{H} 9^{\prime}, \mathrm{H} 10^{\prime}\right), 2.19-2.09\left(\mathrm{~m}, 3 \mathrm{H}, \mathrm{H} 15^{\prime}\right), 1.00\left(\mathrm{t}, J=7.5 \mathrm{~Hz}, 4 \mathrm{H}, \mathrm{H} 16^{\prime}\right)$.

${ }^{13} \mathrm{C}\left\{{ }^{1} \mathrm{H}\right\}$ NMR (101 MHz, $\mathrm{CDCl} 3$ with 0.03\% v/v TMS): $\delta 138.3$ (C6'), 137.0 (C5'), 135.3 (C14'), 129.7 ( $\mathrm{C}_{\mathrm{Ar}}$ or $\left.C_{\mathrm{Ar}^{\prime}}\right), 129.5\left(\mathrm{C} 1^{\prime}\right), 127.13\left(\mathrm{C}_{\mathrm{Ar}}\right.$ or $\left.\mathrm{C}_{\mathrm{Ar}}{ }^{\prime}\right), 127.10\left(\mathrm{C}_{\mathrm{Ar}}\right.$ or $\left.\mathrm{C}_{\mathrm{Ar}}{ }^{\prime}\right), 127.0\left(\mathrm{C} 13^{\prime}\right), 126.7\left(\mathrm{C}_{\mathrm{Ar}}\right.$ or $\left.\mathrm{C}_{\mathrm{Ar}}{ }^{\prime}\right), 126.0\left(\mathrm{C}_{\mathrm{Ar}}\right.$ or $\left.\mathrm{C}_{\mathrm{Ar}}{ }^{\prime}\right), 67.1\left(\mathrm{C} 11^{\prime}, \mathrm{C}^{\prime} 2^{\prime}\right), 59.9\left(\mathrm{C} 8^{\prime}\right), 53.8\left(\mathrm{C}^{\prime}, \mathrm{C} 10^{\prime}\right), 30.9\left(\mathrm{C} 7^{\prime}\right), 21.9\left(\mathrm{C} 15^{\prime}\right), 14.5$ (C16').

HRMS (ESI) $\mathrm{m} / \mathrm{z}[\mathrm{M}+\mathrm{H}]^{+}$calc. for $\mathrm{C}_{16} \mathrm{H}_{24} \mathrm{NO}^{+}$: 246.1852; found: 246.1849 . 


\section{Phenethylamine 5g}<smiles>C/C=C/c1ccccc1CN(CCC)CCC</smiles><smiles>CC/C=C\c1ccccc1CCN(CCC)CCC</smiles>

Starting materials:

Phthalazine (1a) $(98.6 \mathrm{mg}, 750 \mu \mathrm{mol}, 1.00$ equiv); BDLA $(3.80 \mathrm{mg}, 18.6 \mu \mathrm{mol}, 2.49 \mathrm{~mol} \%)$; dipropylamine (7c) $(125 \mu \mathrm{L}, 900 \mu \mathrm{mol}, 1.20$ equiv); butyraldehyde (6a) (100 $\mu \mathrm{L}, 1.09 \mathrm{mmol}$, 1.45 equiv); $\mathrm{NaBH}(\mathrm{OAc})_{3}(328 \mathrm{mg}, 1.50 \mathrm{mmol}, 2.00$ equiv); $\mathrm{AcOH}(90.4 \mu \mathrm{L}, 1.50 \mathrm{mmol}, 2.00$ equiv).

LED:

$470 \mathrm{~nm}$

Temperature:

$50{ }^{\circ} \mathrm{C}$

Purification:

Flash column chromatography ( 2 times) $\left(\mathrm{SiO}_{2}: 20 \mathrm{~g}\right.$, cyclohexane/EtOAc $\left.+1 \% \mathrm{NEt}_{3}, 30: 1\right)$.

Yield: mixture of $E / Z$-isomers $E-5 g / Z-5 g(5.83: 1.00)$

$161 \mathrm{mg}(621 \mu \mathrm{mol}, 83 \%)$; pale yellow oil.

\section{$E-5 g$ :}

${ }^{1} \mathrm{H}$ NMR $\left(400 \mathrm{MHz}, \mathrm{CDCl}_{3}\right.$ with $\left.0.03 \% \mathrm{v} / \mathrm{v} \mathrm{TMS}\right): \delta 7.44-7.39(\mathrm{~m}, 1 \mathrm{H}, \mathrm{H} 4), 7.19-7.09(\mathrm{~m}, 3 \mathrm{H}, \mathrm{H} 1, \mathrm{H} 2$, $\mathrm{H} 3), 6.64(\mathrm{~d}, J=15.6 \mathrm{~Hz}, 1 \mathrm{H}, \mathrm{H} 15), 6.14(\mathrm{dt}, J=15.5,6.5 \mathrm{~Hz}, 1 \mathrm{H}, \mathrm{H} 16), 2.84-2.78(\mathrm{~m}, 2 \mathrm{H}, \mathrm{H} 7), 2.65-$ $2.57(\mathrm{~m}, 2 \mathrm{H}, \mathrm{H} 8), 2.52-2.42(\mathrm{~m}, 4 \mathrm{H}, \mathrm{H} 9, \mathrm{H} 10), 2.25(\mathrm{p}, \mathrm{J}=7.3 \mathrm{~Hz}, 2 \mathrm{H}, \mathrm{H} 17), 1.57-1.43(\mathrm{~m}, 4 \mathrm{H}, \mathrm{H} 11$, $\mathrm{H} 12), 1.11$ (td, $J=7.4,1.0 \mathrm{~Hz}, 3 \mathrm{H}, \mathrm{H} 18), 0.95-0.86(\mathrm{~m}, 6 \mathrm{H}, \mathrm{H} 13, \mathrm{H} 14)$.

${ }^{13} \mathrm{C}\left\{{ }^{1} \mathrm{H}\right\}$ NMR (101 MHz, $\mathrm{CDCl}_{3}$ with 0.03\% v/v TMS): $\delta 137.8$ ( $\mathrm{C}_{\mathrm{Ar}}$ or $\left.\mathrm{C}_{\mathrm{Ar}}{ }^{\prime}\right), 137.0(\mathrm{C} 5), 135.1\left(\mathrm{C}_{\mathrm{Ar}}\right.$ or $\left.\mathrm{C}_{\mathrm{Ar}}{ }^{\prime}\right)$, $134.6\left(C_{\mathrm{Ar}}\right.$ or $\left.\mathrm{C}_{\mathrm{Ar}}{ }^{\prime}\right), 134.5\left(\mathrm{C}_{\mathrm{Ar}}\right.$ or $\left.\mathrm{C}_{\mathrm{Ar}}{ }^{\prime}\right), 130.0(\mathrm{C} 1), 129.7\left(\mathrm{C}_{\mathrm{Ar}}\right.$ or $\left.\mathrm{C}_{\mathrm{Ar}}{ }^{\prime}\right), 129.6\left(\mathrm{C}_{\mathrm{Ar}}\right.$ or $\left.\mathrm{C}_{\mathrm{Ar}}{ }^{\prime}\right), 127.2\left(\mathrm{C}_{\mathrm{Ar}}\right.$ or $\left.\mathrm{C}_{\mathrm{Ar}}{ }^{\prime}\right)$, $127.1\left(\mathrm{C}_{\mathrm{Ar}}\right.$ or $\left.\mathrm{C}_{\mathrm{Ar}}{ }^{\prime}\right), 127.0\left(\mathrm{C}_{\mathrm{Ar}}\right.$ or $\left.\mathrm{C}_{\mathrm{Ar}}{ }^{\prime}\right), 126.46\left(\mathrm{C}_{\mathrm{Ar}}\right.$ or $\left.\mathrm{C}_{\mathrm{Ar}}{ }^{\prime}\right), 126.45\left(\mathrm{C}_{\mathrm{Ar}}\right.$ or $\left.\mathrm{C}_{\mathrm{Ar}}{ }^{\prime}\right), 126.1(\mathrm{C} 4), 125.7\left(\mathrm{C}_{\mathrm{Ar}}\right.$ or $\left.\mathrm{C}_{\mathrm{Ar}}{ }^{\prime}\right), 56.4$ (C9, C10), 55.2 (C8), 31.0 (C7), 26.5 (C17), 20.6 (C11, C12), 13.8 (C18), 12.1 (C13, C14).

\section{$Z-5 g$ :}

${ }^{1} \mathrm{H}$ NMR $\left(400 \mathrm{MHz}, \mathrm{CDCl}_{3}\right.$ with $\left.0.03 \% \mathrm{v} / \mathrm{v} \mathrm{TMS}\right): \delta 7.19-7.09$ (m, 4H, H1', H2', H3', H4'), 6.50 (d, J = $\left.11.4 \mathrm{~Hz}, 1 \mathrm{H}, \mathrm{H} 15^{\prime}\right), 5.72\left(\mathrm{dt}, J=11.4,7.4 \mathrm{~Hz}, 1 \mathrm{H}, \mathrm{H} 16^{\prime}\right), 2.78-2.70\left(\mathrm{~m}, 2 \mathrm{H}, \mathrm{H} 7^{\prime}\right), 2.65-2.57(\mathrm{~m}, 2 \mathrm{H}$, $\left.\mathrm{H} 8^{\prime}\right), 2.52-2.42\left(\mathrm{~m}, 4 \mathrm{H}, \mathrm{H9^{ \prime }}, \mathrm{H} 10^{\prime}\right), 2.15\left(\mathrm{p}, J=7.4 \mathrm{~Hz}, 2 \mathrm{H}, \mathrm{H} 17^{\prime}\right), 1.57-1.43\left(\mathrm{~m}, 4 \mathrm{H}, \mathrm{H} 11^{\prime}, \mathrm{H} 12^{\prime}\right), 1.04$ $-0.96\left(\mathrm{td}, J=7.5,0.9 \mathrm{~Hz}, 3 \mathrm{H}, \mathrm{H} 18^{\prime}\right), 0.95-0.86\left(\mathrm{~m}, 6 \mathrm{H}, \mathrm{H} 13^{\prime}, \mathrm{H} 14^{\prime}\right)$.

${ }^{13} \mathrm{C}\left\{{ }^{1} \mathrm{H}\right\}$ NMR (101 MHz, $\mathrm{CDCl}_{3}$ with $0.03 \% \mathrm{v} / \mathrm{v}$ TMS $): \delta 137.8\left(\mathrm{C}_{\mathrm{Ar}}\right.$ or $\left.\mathrm{C}_{\mathrm{Ar}}{ }^{\prime}\right), 135.1\left(\mathrm{C}_{\mathrm{Ar}}\right.$ or $\left.\mathrm{C}_{\mathrm{Ar}}{ }^{\prime}\right), 134.6\left(\mathrm{C}_{\mathrm{Ar}}\right.$ or $\left.C_{A r}{ }^{\prime}\right), 134.5\left(C_{A r}\right.$ or $\left.C_{A r}{ }^{\prime}\right), 129.7\left(C_{A r}\right.$ or $\left.C_{A r}{ }^{\prime}\right), 129.6\left(C_{A r}\right.$ or $\left.C_{A r}{ }^{\prime}\right), 127.2\left(C_{A r}\right.$ or $\left.C_{A r}{ }^{\prime}\right), 127.1\left(C_{A r}\right.$ or $\left.C_{A r}{ }^{\prime}\right), 127.0$ $\left(\mathrm{C}_{\mathrm{Ar}}\right.$ or $\left.\mathrm{C}_{\mathrm{Ar}}{ }^{\prime}\right), 127.0\left(\mathrm{C}_{\mathrm{Ar}}\right.$ or $\left.\mathrm{C}_{\mathrm{Ar}}{ }^{\prime}\right), 126.5\left(\mathrm{C}_{\mathrm{Ar}}\right.$ or $\left.\mathrm{C}_{\mathrm{Ar}}{ }^{\prime}\right), 125.7\left(\mathrm{C}_{\mathrm{Ar}}\right.$ or $\left.\mathrm{C}_{\mathrm{Ar}}{ }^{\prime}\right), 56.3\left(\mathrm{C} 9^{\prime}, \mathrm{C} 10^{\prime}\right), 54.9\left(\mathrm{C} 8^{\prime}\right), 31.0$ (C7'), 21.9 (C17'), 20.6 (C11', C12'), 14.4 (C18'), 12.1 (C13', C14').

HRMS (ESI) m/z [M+H] calc. for $\mathrm{C}_{18} \mathrm{H}_{30} \mathrm{~N}^{+}:$260.2373; found: 260.2374 . 
Phenethylamine $5 \mathrm{~h}$<smiles>[3H]C/C=C/c1ccccc1CCN(C)CCN(C)C</smiles><smiles>CN(C)CCN(C)CCc1cccc2c1C=C[C+]C=C2</smiles>

Starting materials:

Phthalazine (1a) $(98.6 \mathrm{mg}, 750 \mu \mathrm{mol}, 1.00$ equiv); BDLA (3.80 mg, $18.6 \mu \mathrm{mol}, 2.49 \mathrm{~mol} \%) ; \mathrm{N}, \mathrm{N}, \mathrm{N}^{\prime}-$ trimethylethylenediamine (7d) $(121 \mu \mathrm{L}, 900 \mu \mathrm{mol}, 1.20$ equiv); butyraldehyde (6a) $(100 \mu \mathrm{L}, 1.09 \mathrm{mmol}$, 1.45 equiv); $\mathrm{NaBH}(\mathrm{OAc})_{3}(328 \mathrm{mg}, 1.50 \mathrm{mmol}, 2.00$ equiv); $\mathrm{AcOH}$ (90.4 $\mu \mathrm{L}, 1.50 \mathrm{mmol}, 2.00$ equiv).

LED:

$470 \mathrm{~nm}$

Temperature:

$30^{\circ} \mathrm{C}$

Purification:

Flash column chromatography (2 times) ( $\mathrm{SiO}_{2}: 20 \mathrm{~g}$, cyclohexane/EtOAc/NEt ${ }_{3}, 10: 10: 1$ to 5:5:1).

Yield: mixture of E/Z-isomers E-5h/Z-5h (2.09 : 1.00)

$134 \mathrm{mg}(515 \mu \mathrm{mol}, 69 \%)$; pale yellow oil.

\section{E-5h:}

${ }^{1} \mathrm{H}$ NMR $\left(400 \mathrm{MHz}, \mathrm{CDCl}_{3}\right.$ with $\left.0.03 \% \mathrm{v} / \mathrm{v} \mathrm{TMS}\right): \delta 7.44-7.39(\mathrm{~m}, 1 \mathrm{H}, \mathrm{H} 4), 7.20-7.10(\mathrm{~m}, 3 \mathrm{H}, \mathrm{H} 1, \mathrm{H} 2$, $\mathrm{H} 3), 6.62(\mathrm{~d}, J=15.6 \mathrm{~Hz}, 1 \mathrm{H}, \mathrm{H} 14) 6.14(\mathrm{dt}, J=15.6,6.5 \mathrm{~Hz}, 1 \mathrm{H}, \mathrm{H} 15), 2.88-2.83(\mathrm{~m}, 2 \mathrm{H}, \mathrm{H7}), 2.59-$ $2.51(\mathrm{~m}, 4 \mathrm{H}, \mathrm{H} 8, \mathrm{H} 10), 2.45-2.39(\mathrm{~m}, 2 \mathrm{H}, \mathrm{H} 11), 2.36(\mathrm{~s}, 3 \mathrm{H}, \mathrm{H} 9), 2.25$ (s, 6H, H12, H13), $2.31-2.19$ (m, $2 \mathrm{H}, \mathrm{H} 16), 1.10(\mathrm{t}, J=7.5 \mathrm{~Hz}, 3 \mathrm{H}, \mathrm{H} 17)$.

${ }^{13} \mathrm{C}\left\{{ }^{1} \mathrm{H}\right\}$ NMR (101 MHz, $\mathrm{CDCl}_{3}$ with 0.03\% v/v TMS): $\delta 137.3$ (C6), 137.0 (C5), 134.6 (C15), 129.9 (C1), 127.1 (C2), 126.6 (C3), 126.4 (C14), 126.1 (C4), 59.3 (C8 or C10), 57.60 (C11), 55.6 (C8 or C10), 46.0 (C12, C13), 42.7 (C9), 31.3 (C7), 26.5 (C16), 13.9 (C17).

\section{Z-5h:}

${ }^{1} \mathrm{H}$ NMR (400 MHz, $\mathrm{CDCl}_{3}$ with 0.03\% v/v TMS): $\delta 7.20-7.10$ (m, 4H, H1', H2', H3', H4'), 6.48 (dt, J= 11.4, $\left.1.5 \mathrm{~Hz}, 1 \mathrm{H}, \mathrm{H} 14^{\prime}\right), 5.72\left(\mathrm{dt}, \mathrm{J}=11.4,7.4 \mathrm{~Hz}, 1 \mathrm{H}, \mathrm{H} 15^{\prime}\right), 2.80-2.75\left(\mathrm{~m}, 2 \mathrm{H}, \mathrm{H} 7^{\prime}\right), 2.59-2.51(\mathrm{~m}, 4 \mathrm{H}$, $\left.\mathrm{H} 8^{\prime}, \mathrm{H} 10^{\prime}\right), 2.45-2.39\left(\mathrm{~m}, 2 \mathrm{H}, \mathrm{H} 11^{\prime}\right), 2.33\left(\mathrm{~s}, 3 \mathrm{H}, \mathrm{H}^{\prime}\right), 2.25\left(\mathrm{~s}, 6 \mathrm{H}, \mathrm{H} 12^{\prime}, \mathrm{H} 13^{\prime}\right), 2.15$ (qd, J = 7.5, $1.6 \mathrm{~Hz}$, $\left.2 \mathrm{H}, \mathrm{H} 16^{\prime}\right), 0.99\left(\mathrm{t}, J=7.5 \mathrm{~Hz}, 3 \mathrm{H}, \mathrm{H} 17^{\prime}\right)$.

${ }^{13} \mathrm{C}\left\{{ }^{1} \mathrm{H}\right\}$ NMR (101 MHz, CDCl 3 with 0.03\% v/v TMS): $\delta 138.7$ (C6'), 136.8 (C5'), 135.2 (C15'), 129.7 (C1'

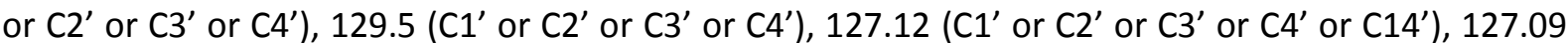

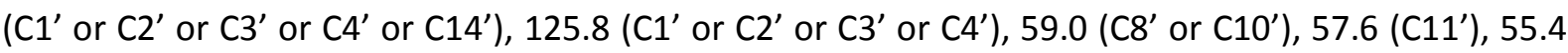
(C8' or $\left.\mathrm{C} 10^{\prime}\right), 46.0\left(\mathrm{C} 12^{\prime}, \mathrm{C} 13^{\prime}\right), 42.6\left(\mathrm{C} 9^{\prime}\right), 31.2\left(\mathrm{C} 7^{\prime}\right), 21.9$ (C16'), 14.5 (C17').

HRMS (ESI) m/z [M+H] $]^{+}$calc. for $\mathrm{C}_{17} \mathrm{H}_{29} \mathrm{~N}_{2}{ }^{+}:$261.2325; found: 261.2322 . 
Phenethylamine 5i
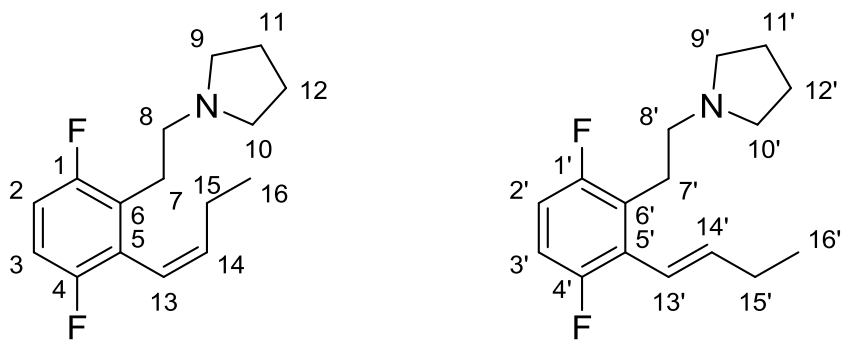

Starting materials:

5,8-Difluorophthalazine (1b) (62.3 mg, $375 \mu \mathrm{mol}, 1.00$ equiv); BDLA (1.90 mg, $9.30 \mu \mathrm{mol}, 2.49 \mathrm{~mol} \%$ ); pyrrolidine (7a) $(37.3 \mu \mathrm{L}, 450 \mu \mathrm{mol}, 1.20$ equiv); butyraldehyde $(50 \mu \mathrm{L}, 544 \mu \mathrm{mol}, 1.45$ equiv); $\mathrm{NaBH}(\mathrm{OAc})_{3}(164 \mathrm{mg}, 735 \mu \mathrm{mol}, 2.00$ equiv); $\mathrm{AcOH}$ (45.2 $\mu \mathrm{L}, 750 \mu \mathrm{mol}, 2.00$ equiv).

LED:

$448 \mathrm{~nm}$

Temperature:

$-15^{\circ} \mathrm{C}$ to $10^{\circ} \mathrm{C}$

Purification:

Flash column chromatography (2 times) $\left(\mathrm{SiO}_{2}: 20 \mathrm{~g}\right.$, cyclohexane/EtOAc $\left.+1 \% \mathrm{NEt}_{3}, 8: 1\right)$.

Yield: mixture of E/Z-isomers E-5h/Z-5h (1.00 : 5.71)

$58.0 \mathrm{mg}$ (219 mmol, 58\%); pale yellow oil.

\section{E-5i:}

${ }^{1} \mathrm{H} \mathrm{NMR}\left(400 \mathrm{MHz}, \mathrm{CDCl}_{3}\right.$ with $\left.0.03 \% \mathrm{v} / \mathrm{v} \mathrm{TMS}\right): \delta 6.93-6.78(\mathrm{~m}, 2 \mathrm{H}, \mathrm{H} 2, \mathrm{H} 3), 6.30-6.25(\mathrm{~m}, 2 \mathrm{H}, \mathrm{H} 13$, $\mathrm{H} 14), 2.97-2.88(\mathrm{~m}, 2 \mathrm{H}, \mathrm{H} 7), 2.64-2.47(\mathrm{~m}, 6 \mathrm{H}, \mathrm{H} 8, \mathrm{H} 9, \mathrm{H} 10), 2.32-2.22(\mathrm{~m}, 2 \mathrm{H}, \mathrm{H} 15), 1.86-1.73$ (m, $4 \mathrm{H}, \mathrm{H} 11, \mathrm{H} 12), 1.10(\mathrm{t}, J=7.5 \mathrm{~Hz}, 3 \mathrm{H}, \mathrm{H} 16)$.

${ }^{13} \mathrm{C}\left\{{ }^{1} \mathrm{H}\right\}$ NMR (101 MHz, $\mathrm{CDCl}_{3}$ with $0.03 \%$ v/v TMS): $\delta 157.3$ (dd, J = 240.3, $2.5 \mathrm{~Hz}, \mathrm{C} 1$, detected by hmbc experiment), 155.6 (dd, J = 240.2, $2.2 \mathrm{~Hz}, \mathrm{C} 4$, detected by hmbc experiment), 140.8 (d, J=8.8 Hz, C14), 126.7 (C5), 119.3 (d, $J=2.7 \mathrm{~Hz}, 13$ ), 114.1 (dd, $J=27.1,9.9 \mathrm{~Hz}, \mathrm{C} 2), 113.6$ (dd, $J=26.0,9.4 \mathrm{~Hz}, \mathrm{C} 3$ ), 55.8 (C8), 54.3 (C9, C10) 27.1 (C15), 25.8 (C7), 23.6 (C11, C12), 13.6 (C16).

Z-5i:

${ }^{1} \mathrm{H} \mathrm{NMR}\left(400 \mathrm{MHz}, \mathrm{CDCl}_{3}\right.$ with $\left.0.03 \% \mathrm{v} / \mathrm{v} \mathrm{TMS}\right): \delta 6.93-6.78\left(\mathrm{~m}, 2 \mathrm{H}, \mathrm{H}^{\prime}, \mathrm{H}^{\prime}\right), 6.13(\mathrm{~d}, \mathrm{~J}=11.2 \mathrm{~Hz}, 1 \mathrm{H}$, $\left.\mathrm{H} 13^{\prime}\right), 5.89\left(\mathrm{dt}, J=11.2,7.3 \mathrm{~Hz}, 1 \mathrm{H}, \mathrm{H} 14^{\prime}\right), 2.86-2.79\left(\mathrm{~m}, 2 \mathrm{H}, \mathrm{H} 7^{\prime}\right), 2.64-2.47\left(\mathrm{~m}, 6 \mathrm{H}, \mathrm{H} 8^{\prime}, \mathrm{H} 9^{\prime}, \mathrm{H} 10^{\prime}\right)$, $1.94\left(\mathrm{p}, J=7.4 \mathrm{~Hz}, 2 \mathrm{H}, \mathrm{H} 15^{\prime}\right), 1.86-1.73\left(\mathrm{~m}, 4 \mathrm{H}, \mathrm{H} 11^{\prime}, \mathrm{H} 12^{\prime}\right), 0.96\left(\mathrm{t}, J=7.5 \mathrm{~Hz}, 1 \mathrm{H}, \mathrm{H} 16^{\prime}\right)$.

${ }^{13} \mathrm{C}\left\{{ }^{1} \mathrm{H}\right\}$ NMR (101 MHz, $\mathrm{CDCl}_{3}$ with 0.03\% v/v TMS): $\delta 157.3$ (dd, $\left.J=240.3,2.5 \mathrm{~Hz}, \mathrm{C} 1^{\prime}\right), 155.6$ (dd, $J=$ 240.2, 2.2 Hz, C4'), 139.14 (d, J = 1.4 Hz, C14'), 127.64 (dd, $\left.J=17.7,3.3 \mathrm{~Hz}, \mathrm{C} 6^{\prime}\right), 126.2$ (dd, $J=19.1$, $\left.4.9 \mathrm{~Hz}, \mathrm{C} 5^{\prime}\right) 118.8\left(\mathrm{~d}, J=2.1 \mathrm{~Hz}, \mathrm{C} 13^{\prime}\right), 114.5$ (dd, J = 25.7, 9.0 Hz, C2'), 113.8 (dd, J=26.3, 9.2 Hz, C3'), $55.5\left(\mathrm{C} 8^{\prime}\right), 54.2\left(\mathrm{C}^{\prime}, \mathrm{C} 10^{\prime}\right), 26.3\left(\mathrm{C} 7^{\prime}\right) 23.6\left(\mathrm{C} 11^{\prime}, \mathrm{C} 12^{\prime}\right), 22.8\left(\mathrm{~d}, J=2.4 \mathrm{~Hz}, \mathrm{C} 15^{\prime}\right), 13.5$ (C16').

HRMS (ESI) m/z [M+H] calc. for $\mathrm{C}_{16} \mathrm{H}_{22} \mathrm{~F}_{2} \mathrm{~N}^{+}$: 266.1715; found: 266.1713 . 
Phenethylamine $5 \mathbf{j}$<smiles>CC/C=C/c1ccccc1CCN1CCCC1</smiles><smiles>CC/C=C\c1ccccc1CCN1CCCC1</smiles>

Starting materials:

6-(Trifluoromethyl)phthalazine (1c) $(149 \mathrm{mg}, 750 \mu \mathrm{mol}, 1.00$ equiv); BDLA (3.80 mg, $18.6 \mu \mathrm{mol}$, $2.49 \mathrm{~mol} \%)$; pyrrolidine (7a) $(74.7 \mu \mathrm{L}, 900 \mu \mathrm{mol}, 1.20$ equiv); butyraldehyde (100 $\mu \mathrm{L}, 1.09 \mathrm{mmol}$, 1.45 equiv); $\mathrm{NaBH}(\mathrm{OAc})_{3}(328 \mathrm{mg}, 1.50 \mathrm{mmol}, 2.00$ equiv); $\mathrm{AcOH}(90.4 \mu \mathrm{L}, 1.50 \mathrm{mmol}, 2.00$ equiv).

LED:

$448 \mathrm{~nm}$

Temperature:

$-10{ }^{\circ} \mathrm{C}$ to $0^{\circ} \mathrm{C}$

Purification:

Flash column chromatography (2 times) $\left(\mathrm{SiO}_{2}: 20 \mathrm{~g}\right.$, cyclohexane/EtOAc $\left.+1 \% \mathrm{NEt}_{3}, 8: 1\right)$.

Yield: mixture of $E / Z$-isomers $E-5 \mathrm{j} / Z-5 \mathrm{j}(9.00: 1.00)$

$67.0 \mathrm{mg}(225 \mu \mathrm{mol}, 30 \%)$; pale yellow oil.
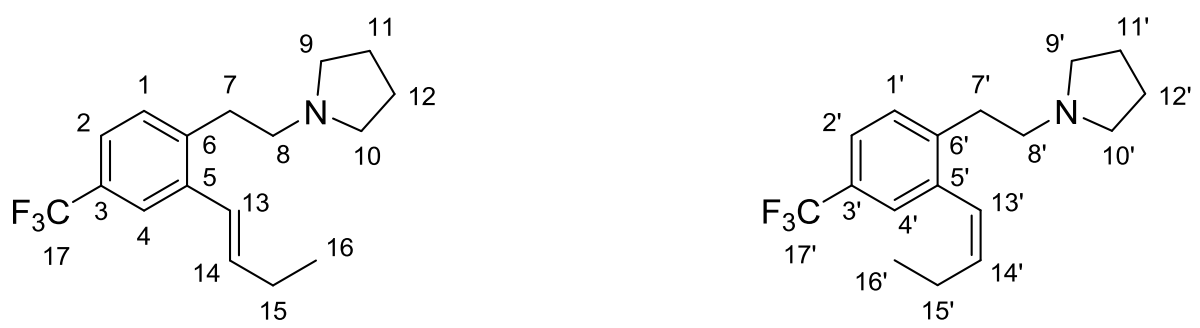

$E-5$; :

${ }^{1} \mathrm{H}$ NMR $\left(400 \mathrm{MHz}, \mathrm{CDCl}_{3}\right.$ with $\left.0.03 \% \mathrm{v} / \mathrm{v} \mathrm{TMS}\right): \delta 7.64(\mathrm{~d}, J=1.9 \mathrm{~Hz}, 1 \mathrm{H}, \mathrm{H} 4), 7.37$ (dd, $J=8.1,1.9 \mathrm{~Hz}$, $1 \mathrm{H}, \mathrm{H} 2$ ), 7.25 (d, $J=7.8 \mathrm{~Hz}, 1 \mathrm{H}, \mathrm{H} 1), 6.64(\mathrm{dt}, J=15.6,1.7 \mathrm{~Hz}, 1 \mathrm{H}, \mathrm{H} 13), 6.21(\mathrm{dt}, J=15.6,6.5 \mathrm{~Hz}, 1 \mathrm{H}$, H14), $2.97-2.90(m, 2 H, H 7), 2.66-2.54(m, 6 H, H 8, H 9, H 10), 2.31-2.22(m, 2 H, H 15), 1.87-1.78$ (m, 4H, H11, H12), $1.11(\mathrm{t}, J=7.5 \mathrm{~Hz}, 3 \mathrm{H}, \mathrm{H} 16)$.

${ }^{13} \mathrm{C}\left\{{ }^{1} \mathrm{H}\right\}$ NMR (101 MHz, $\mathrm{CDCl}_{3}$ with $\left.0.03 \% \mathrm{v} / \mathrm{v} \mathrm{TMS}\right): \delta 141.1$ (q, $J=1.2 \mathrm{~Hz}, \mathrm{C} 6$, only visible as doublet due to low intensity), 137.7 (C5), 136.5 (C14), 130.2 (C1), 128.9 (q, $J=32.0 \mathrm{~Hz}, \mathrm{C} 3$ ), 125.4 (C13), 124.5 (q, $J=272.0 \mathrm{~Hz}, \mathrm{C} 17), 123.5$ (q, J = 3.8 Hz, C2), 122.9 (q, J = 3.8 Hz, C4), 57.1 (C8), 54.4 (C9, C10), 33.2 (C7), 26.5 (C15), 23.6 (C11, C12), 13.8 (C16).

\section{Z-5j:}

${ }^{1} \mathrm{H}$ NMR (400 MHz, $\mathrm{CDCl}_{3}$ with $0.03 \%$ v/v TMS): $\delta 7.42$ (dd, $J=8.1,2.0 \mathrm{~Hz}, 1 \mathrm{H}, \mathrm{Hz}$ '), $7.38(\mathrm{~s}, 1 \mathrm{H}, \mathrm{H} 4$ ', detected by HMQC experiment), $7.30\left(\mathrm{~d}, J=8.0 \mathrm{~Hz}, 1 \mathrm{H}, \mathrm{H} 1^{\prime}\right), 6.47\left(\mathrm{~d}, J=11.5 \mathrm{~Hz}, 1 \mathrm{H}, \mathrm{H} 13^{\prime}\right), 5.79$ (dt, $J$ $\left.=11.4,7.4 \mathrm{~Hz}, 1 \mathrm{H}, \mathrm{H} 14^{\prime}\right), 2.89-2.82\left(\mathrm{~m}, 2 \mathrm{H}, \mathrm{H} 7^{\prime}\right), 2.66-2.54\left(\mathrm{~m}, 6 \mathrm{H}, \mathrm{H} 8^{\prime}, \mathrm{H} 9^{\prime}, \mathrm{H} 10^{\prime}\right), 2.11$ (pd, J = 7.5, $\left.1.7 \mathrm{~Hz}, 2 \mathrm{H}, \mathrm{H} 15^{\prime}\right), 1.87-1.78\left(\mathrm{~m}, 4 \mathrm{H}, \mathrm{H} 11^{\prime}, \mathrm{H} 12^{\prime}\right), 1.00\left(\mathrm{t}, J=7.5 \mathrm{~Hz}, 3 \mathrm{H}, \mathrm{H} 16^{\prime}\right)$.

${ }^{13} \mathrm{C}\left\{{ }^{1} \mathrm{H}\right\}$ NMR (101 MHz, $\mathrm{CDCl}_{3}$ with $0.03 \%$ v/v TMS): $\delta 142.8$ (C6'), 137.4 (C5'), 136.6 (C14'), 129.8 (C1'), 126.4 (q, $J=3.6 \mathrm{~Hz}, \mathrm{C} 4^{\prime}$, only visible as doublet due to low intensity), 126.0 (C13') 123.8 (q, $J=3.7 \mathrm{~Hz}$, $\mathrm{C2}^{\prime}$, only visible as doublet due to low intensity), $56.9\left(\mathrm{C} 8^{\prime}\right), 54.3\left(\mathrm{C} 9^{\prime}, \mathrm{C} 10^{\prime}\right), 33.3\left(\mathrm{C} 7^{\prime}\right), 23.6$ (C11', C12'), 21.9 (C15'), 14.3 (C16'). 
HRMS (ESI) $\mathrm{m} / \mathrm{z}$ [M+H] $]^{+}$calc. for $\mathrm{C}_{17} \mathrm{H}_{23} \mathrm{~F}_{3} \mathrm{~N}^{+}:$: 298.1777; found: 298.1780 .

Yield: mixture of $E / Z$-isomers $E-5 j^{\prime} / Z-5 j^{\prime}(3.12: 1.00)$

$54.0 \mathrm{mg}(182 \mu \mathrm{mol}, 24 \%)$; pale yellow oil.
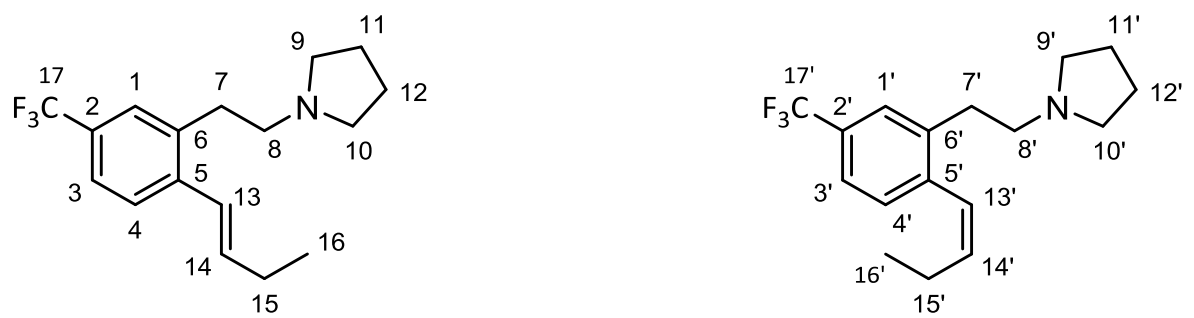

$E-5 j^{\prime}:$

${ }^{1} \mathrm{H} \mathrm{NMR}\left(400 \mathrm{MHz}, \mathrm{CDCl}_{3}\right.$ with $\left.0.03 \% \mathrm{v} / \mathrm{v} \mathrm{TMS}\right): \delta 7.50(\mathrm{~d}, J=8.5 \mathrm{~Hz}, 1 \mathrm{H}, \mathrm{H} 4), 7.45-7.36(\mathrm{~m}, 2 \mathrm{H}, \mathrm{H} 1$, $\mathrm{H} 3), 6.65(\mathrm{~d}, J=15.7 \mathrm{~Hz}, 1 \mathrm{H}, \mathrm{H} 13), 6.22(\mathrm{dt}, J=15.6,6.6 \mathrm{~Hz}, 1 \mathrm{H}, \mathrm{H} 14), 2.98-2.91(\mathrm{~m}, 2 \mathrm{H}, \mathrm{H} 7), 2.68-$ $2.55(\mathrm{~m}, 6 \mathrm{H}, \mathrm{H} 8, \mathrm{H} 9, \mathrm{H} 10), 2.32-2.21(\mathrm{~m}, 2 \mathrm{H}, \mathrm{H} 15), 1.88-1.78(\mathrm{~m}, 4 \mathrm{H}, \mathrm{H} 11, \mathrm{H} 12), 1.11$ (t, J = $7.4 \mathrm{~Hz}$, $3 \mathrm{H}, \mathrm{H} 16)$.

${ }^{13} \mathrm{C}\left\{{ }^{1} \mathrm{H}\right\}$ NMR (101 MHz, CDCl 3 with $0.03 \%$ v/v TMS): $\delta 140.6$ (q, $J=1.5 \mathrm{~Hz}, \mathrm{C} 5$, only visible as doublet due to low intensity), 137.7 (C6), 137.1 (C14), 128.9 (q, $J=32.2 \mathrm{~Hz}, \mathrm{C2}$ ), 126.5 (q, $J=3.8 \mathrm{~Hz}, \mathrm{C} 1$ ), 126.4 (C4), 125.4 (C13), 124.5 (q, J = 271.8 Hz, C17), 123.3 (q, J=3.8 Hz, C3), 57.2 (C8), 54.3 (C9, C10), 33.1 (C7), 26.5 (C15), 23.60 (C11, C12), 13.7 (C16).

Z-5j':

${ }^{1} \mathrm{H}$ NMR (400 MHz, $\mathrm{CDCl}_{3}$ with $\left.0.03 \% \mathrm{v} / \mathrm{v} \mathrm{TMS}\right): \delta 7.45-7.36\left(\mathrm{~m}, 2 \mathrm{H}, \mathrm{H}^{\prime}, \mathrm{H} 3^{\prime}\right), 7.24(\mathrm{~d}, J=8.0 \mathrm{~Hz}, 1 \mathrm{H}$, $\left.\mathrm{H} 4^{\prime}\right), 6.48\left(\mathrm{~d}, J=11.4 \mathrm{~Hz}, 1 \mathrm{H}, \mathrm{H} 13^{\prime}\right), 5.80\left(\mathrm{dt}, J=11.5,7.5 \mathrm{~Hz}, 1 \mathrm{H}, \mathrm{H} 14^{\prime}\right), 2.90-2.83\left(\mathrm{~m}, 1 \mathrm{H}, \mathrm{H} 7^{\prime}\right), 2.68-$ $2.55\left(\mathrm{~m}, 6 \mathrm{H}, \mathrm{H} 8^{\prime}, \mathrm{H9^{ \prime }}, \mathrm{H} 10^{\prime}\right), 2.11\left(\mathrm{pd}, J=7.5,1.7 \mathrm{~Hz}, 2 \mathrm{H}, \mathrm{H} 15^{\prime}\right), 1.88-1.78\left(\mathrm{~m}, 4 \mathrm{H}, \mathrm{H} 11^{\prime}, \mathrm{H} 12^{\prime}\right), 0.99$ (t, $\left.J=7.5 \mathrm{~Hz}, 1 \mathrm{H}, \mathrm{H} 16^{\prime}\right)$.

${ }^{13} \mathrm{C}\left\{{ }^{1} \mathrm{H}\right\}$ NMR (101 MHz, CDCl 3 with $0.03 \% \mathrm{v} / \mathrm{v}$ TMS): $\delta 140.5$ (q, $J=1.2 \mathrm{~Hz}, \mathrm{C5}^{\prime}$, only visible as doublet due to low intensity), 139.4 (C6 $\left.6^{\prime}\right), 136.7$ (C14'), $123.0\left(C 4^{\prime}\right), 129.1$ (q, $J=32.1 \mathrm{~Hz}, C 2^{\prime}$, only visible as doublet due to low intensity), 126.1 (q, $J=3.9 \mathrm{~Hz}, \mathrm{C1}^{\prime}$, only visible as doublet due to low intensity), 126.0 (C13'), 122.7 ( $\mathrm{q}, \mathrm{J}=3.9 \mathrm{~Hz}, \mathrm{C} 3^{\prime}$, only visible as doublet due to low intensity), 56.9 (C8'), 54.3 (C9', C10'), 33.2 (C7'), 23.6 (C11', C12'), 21.9 (C15'), 14.3 (C16').

HRMS (ESI) m/z [M+H] calc. for $\mathrm{C}_{17} \mathrm{H}_{23} \mathrm{~F}_{3} \mathrm{~N}^{+}$: 298.1777; found: 298.1780 . 
Phenethylamine 5k
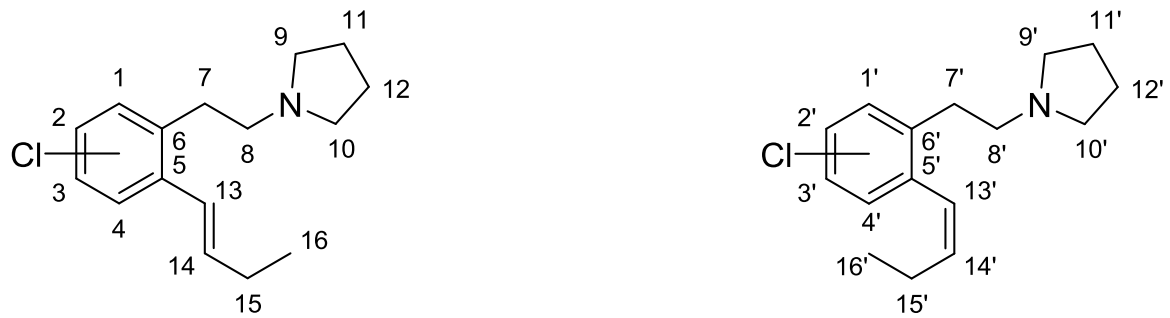

Starting materials:

6-Chlorophthalazine (1d) (123 mg, $750 \mu \mathrm{mol}, 1.00$ equiv); BDLA (3.80 mg, $18.6 \mu \mathrm{mol}, 2.49 \mathrm{~mol} \%$ ); pyrrolidine (7a) (74.7 $\mu \mathrm{L}, 900 \mu \mathrm{mol}, 1.20$ equiv); butyraldehyde (6a) (100 $\mu \mathrm{L}, 1.09 \mathrm{mmol}, 1.45$ equiv); $\mathrm{NaBH}(\mathrm{OAc})_{3}$ (328 mg, $1.50 \mathrm{mmol}, 2.00$ equiv); $\mathrm{AcOH}$ (90.4 $\mu \mathrm{L}, 1.50 \mathrm{mmol}, 2.00$ equiv).

LED:

$448 \mathrm{~nm}$

Temperature:

$-15^{\circ} \mathrm{C}$ to $10^{\circ} \mathrm{C}$

Purification:

Flash column chromatography (2 times) $\left(\mathrm{SiO}_{2}: 20 \mathrm{~g}\right.$, cyclohexane/EtOAc $\left.+1 \% \mathrm{NEt}_{3}, 3: 1\right)$.

Yield: mixture of $E / Z-E-5 k / Z-5 k(5.73: 1.00)$

and the corresponding constitutional isomers $\left(\boldsymbol{E}-\mathbf{5} \mathbf{k}^{\prime} / \mathbf{Z}-\mathbf{5} \mathbf{k}^{\prime}\right)$.

$142 \mathrm{mg}(537 \mu \mathrm{mol}, 72 \%)$; pale yellow oil.

NMR spectra could not be assigned unambiguously

$E-5 k$ and $E-5 k^{\prime}$ :

${ }^{1} \mathrm{H}$ NMR (400 MHz, CDCl 3 with 0.03\% v/v TMS): $\delta 7.38$ (d, $\left.J=2.1 \mathrm{~Hz}, 1 \mathrm{H}, \mathrm{H} 4\right), 7.33$ (d, $\left.J=8.8 \mathrm{~Hz}, 1 \mathrm{H}, \mathrm{H} 4\right)$, $7.15-7.03(\mathrm{~m}, 6 \mathrm{H}, \mathrm{H1}, \mathrm{H} 2, \mathrm{H} 3), 6.56(\mathrm{dt}, J=15.6,1.7 \mathrm{~Hz}, 2 \mathrm{H}, \mathrm{H} 13), 6.13(\mathrm{ddt}, J=15.6,13.5,6.6 \mathrm{~Hz}, 2 \mathrm{H}$, $\mathrm{H} 14), 2.90-2.80(\mathrm{~m}, 4 \mathrm{H}, \mathrm{H7}), 2.65-2.52(\mathrm{~m}, 12 \mathrm{H}, \mathrm{H} 8, \mathrm{H9}, \mathrm{H} 10), 2.30-2.17(\mathrm{~m}, 4 \mathrm{H}, \mathrm{H} 15), 1.86-1.77$ (m, 8H, H11, H12), 1.09 (td, J = 7.4, $1.9 \mathrm{~Hz}, 6 \mathrm{H}, \mathrm{H} 16)$.

${ }^{13} \mathrm{C}\left\{{ }^{1} \mathrm{H}\right\}$ NMR $\left(101 \mathrm{MHz}, \mathrm{CDCl}_{3}\right.$ with $\left.0.03 \% \mathrm{v} / \mathrm{v} \mathrm{TMS}\right): \delta 139.0\left(\mathrm{C}_{\mathrm{Ar}}\right), 138.7\left(\mathrm{C}_{\mathrm{Ar}}\right), 135.91(\mathrm{C} 14), 135.7\left(\mathrm{C}_{\mathrm{Ar}}\right)$, $135.5\left(C_{\mathrm{Ar}}\right), 135.2(\mathrm{C} 14), 132.4\left(\mathrm{C}_{\mathrm{Ar}}\right), 132.2\left(\mathrm{C}_{\mathrm{Ar}}\right), 131.2\left(\mathrm{C}_{\mathrm{Ar}}\right), 129.6\left(\mathrm{C}_{\mathrm{Ar}}\right), 127.35(\mathrm{C} 4), 126.9\left(\mathrm{C}_{\mathrm{Ar}}\right), 126.6$ $\left(C_{\mathrm{Ar}}\right), 125.90$ (C4), 125.4 (C13), 57.5 (C8), 57.3 (C8), 54.4 (C9, C10), 33.1 (C7), 32.7 (C7), 26.45 (C15), 26.42 (C15), 23.6 (C11, C12), 13.83 (C16), 13.79 (C16).

\section{Z-5k and Z-5k':}

${ }^{1} \mathrm{H}$ NMR $\left(400 \mathrm{MHz}, \mathrm{CDCl}_{3}\right.$ with $\left.0.03 \% \mathrm{v} / \mathrm{v} \mathrm{TMS}\right): \delta 7.18\left(\mathrm{~d}, J=2.2 \mathrm{~Hz}, 1 \mathrm{H}, \mathrm{H}_{\mathrm{Ar}}{ }^{\prime}\right), 7.15-7.03\left(\mathrm{~m}, 5 \mathrm{H}, \mathrm{H}_{\mathrm{Ar}}{ }^{\prime}\right)$, $6.41\left(\mathrm{dd}, J=11.4,1.7 \mathrm{~Hz}, 2 \mathrm{H}, \mathrm{H} 13^{\prime}\right), 5.73\left(\mathrm{dtd}, J=12.2,7.4,4.9 \mathrm{~Hz}, 2 \mathrm{H}, \mathrm{H} 14^{\prime}\right), 2.82-2.73\left(\mathrm{~m}, 4 \mathrm{H}, \mathrm{H} 7^{\prime}\right)$, $2.65-2.52\left(\mathrm{~m}, 12 \mathrm{H}, \mathrm{H} 8^{\prime}, \mathrm{H} 9^{\prime}, \mathrm{H} 10^{\prime}\right), 2.19-2.03\left(\mathrm{~m}, 4 \mathrm{H}, \mathrm{H} 15^{\prime}\right), 1.86-1.77\left(\mathrm{~m}, 8 \mathrm{H}, \mathrm{H} 11^{\prime}, \mathrm{H} 12^{\prime}\right), 0.99$ (td, $\left.J=7.5,4.9 \mathrm{~Hz}, 6 \mathrm{H}, \mathrm{H} 16^{\prime}\right)$.

${ }^{13} \mathrm{C}\left\{{ }^{1} \mathrm{H}\right\}$ NMR (101 MHz, CDCl 3 with 0.03\% v/v TMS): $\delta 140.6\left(\mathrm{C}_{\mathrm{Ar}}{ }^{\prime}\right), 138.5\left(\mathrm{C}_{\mathrm{Ar}}{ }^{\prime}\right), 137.2\left(\mathrm{C}_{\mathrm{Ar}}{ }^{\prime}\right), 136.2\left(\mathrm{C} 14^{\prime}\right)$, $135.87\left(\mathrm{C}^{\prime} 4^{\prime}\right), 132.48\left(\mathrm{C}_{\mathrm{Ar}}{ }^{\prime}\right), 131.4\left(\mathrm{C}_{\mathrm{Ar}}{ }^{\prime}\right), 130.9\left(\mathrm{C}_{\mathrm{Ar}}{ }^{\prime}\right), 130.7\left(\mathrm{C}_{\mathrm{Ar}}{ }^{\prime}\right), 129.33\left(\mathrm{C}_{\mathrm{Ar}}{ }^{\prime}\right), 129.29\left(\mathrm{C}_{\mathrm{Ar}}{ }^{\prime}\right), 127.0\left(\mathrm{C}_{\mathrm{z}}{ }^{\prime}\right)$, 126.03 (C13' or CAr'), 126.00 (C13' or CAr'), 125.93 (C13' or CAr'), 57.2 (C8'), 57.0 (C8'), 54.31 (C9', C10'), 33.25 (C7'), 32.84 (C7'), 23.59 (C11', C12'), 21.9 (C15'), 14.34 (C16'), 14.30 (C16')

HRMS (ESI) m/z [M+H] calc. for $\mathrm{C}_{16} \mathrm{H}_{23} \mathrm{CIN}^{+}$: 264.1514; found: 264.1514 . 
Phenethylamine 5I
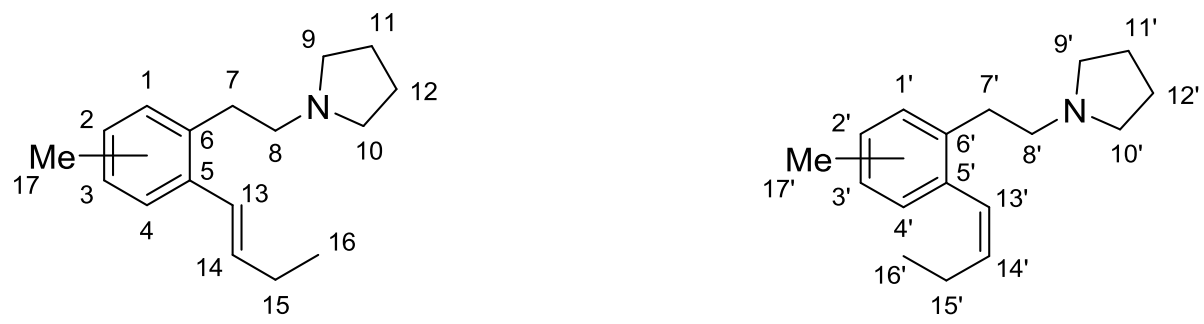

Starting materials:

6-Methylphthalazine (1e) (149 mg, $750 \mu \mathrm{mol}, 1.00$ equiv); BDLA (3.80 mg, $18.6 \mu \mathrm{mol}, 2.49 \mathrm{~mol} \%$ ); pyrrolidine (7a) $(74.7 \mu \mathrm{L}, 900 \mu \mathrm{mol}, 1.20$ equiv); butyraldehyde (6a) $(100 \mu \mathrm{L}, 1.09 \mathrm{mmol}, 1.45$ equiv); $\mathrm{NaBH}(\mathrm{OAc})_{3}$ (328 mg, $1.50 \mathrm{mmol}, 2.00$ equiv); $\mathrm{AcOH}$ (90.4 $\mu \mathrm{L}, 1.50 \mathrm{mmol}, 2.00$ equiv).

LED:

$448 \mathrm{~nm}$

Temperature:

$40^{\circ} \mathrm{C}$

Purification:

Flash column chromatography (2 times) $\left(\mathrm{SiO}_{2}: 20 \mathrm{~g}\right.$, cyclohexane/EtOAc $\left.+5 \% \mathrm{NEt}_{3}, 20: 1\right)$.

Yield: mixture of $E / Z$-isomers $E-5 I / Z-5 I ~(1.71: 1.00)$

and the corresponding constitutional isomers $\left(E-\left.5\right|^{\prime} / Z-\left.5\right|^{\prime}\right)$.

$142 \mathrm{mg}$ (584 $\mu \mathrm{mol}, 78 \%$ ); pale yellow oil.

NMR spectra could not be assigned unambiguously

${ }^{1} \mathrm{H}$ NMR $\left(400 \mathrm{MHz}, \mathrm{CDCl}_{3}\right.$ with $\left.0.03 \% \mathrm{v} / \mathrm{v} \mathrm{TMS}\right): \delta 7.33\left(\mathrm{~d}, J=7.7 \mathrm{~Hz}, 1 \mathrm{H}, \mathrm{H}_{\mathrm{Ar}}\right.$ or $\left.\mathrm{H}_{\mathrm{Ar}}{ }^{\prime}\right), 7.25\left(\mathrm{~s}, 1 \mathrm{H}, \mathrm{H}_{\mathrm{Ar}}\right.$ or $\left.\mathrm{H}_{\mathrm{Ar}}{ }^{\prime}\right), 7.12-6.93\left(\mathrm{~m}, 10 \mathrm{H}, \mathrm{H}_{\mathrm{Ar}}\right.$ or $\left.\mathrm{H}_{\mathrm{Ar}}{ }^{\prime}\right), 6.62(\mathrm{dd}, J=15.7,5.3 \mathrm{~Hz}, 2 \mathrm{H}, \mathrm{H} 13), 6.47$ (d, J = 11.4 Hz, 2H, H13'), $6.13(\mathrm{dt}, J=15.5,6.6 \mathrm{~Hz}, 2 \mathrm{H}, \mathrm{H} 14), 5.69$ (dtd, $\left.J=11.7,7.3,4.7 \mathrm{~Hz}, 2 \mathrm{H}, \mathrm{H} 14^{\prime}\right), 2.91-2.83(\mathrm{~m}, 4 \mathrm{H}, \mathrm{H} 7)$, $2.84-2.75\left(\mathrm{~m}, 4 \mathrm{H}, \mathrm{H} 7^{\prime}\right), 2.66-2.53\left(\mathrm{~m}, 24 \mathrm{H}, \mathrm{H} 8, \mathrm{H9}, \mathrm{H} 10, \mathrm{H} 8^{\prime}, \mathrm{H} 9^{\prime}, \mathrm{H} 1 \mathrm{O}^{\prime}\right), 2.31$ (overlapping singlets, look like a t, $\left.J=4.1 \mathrm{~Hz}, 12 \mathrm{H}, \mathrm{H} 17, \mathrm{H} 17^{\prime}\right), 2.30-2.17(\mathrm{~m}, 4 \mathrm{H}, \mathrm{H} 15), 2.19-2.10\left(\mathrm{~m}, 4 \mathrm{H}, \mathrm{H} 15^{\prime}\right), 1.88-1.75$ (m, 16H, H11, H12, H11', H12'), 1.09 (td, J = 7.5, 3.0 Hz, 6H, H16), 0.99 (td, J = 7.5, 1.8 Hz, 6H, H16').

${ }^{13} \mathrm{C}\left\{{ }^{1} \mathrm{H}\right\}$ NMR (101 MHz, CDCl 3 with 0.03\% v/v TMS): $\delta$ 138.5, 137.1, 136.7, 136.6, 135.9, 135.6, 135.3, 135.0, 134.9, 134.3, 134.3, 134.1, 133.8, 133.7, 130.6, 130.3, 130.2, 129.8, 129.6, 129.4, 127.9, 127.8, $127.4,127.2,126.9,126.64,126.59,126.4,126.2,125.9,57.9,57.8,57.6,57.5,54.4,54.3,33.4,33.2$, $33.0,32.9,26.5,26.5,23.6,21.9,21.24,21.20,21.17,14.49,14.47,14.00,13.99$.

HRMS (ESI) $\mathrm{m} / \mathrm{z}[\mathrm{M}+\mathrm{H}]^{+}$calc. for $\mathrm{C}_{17} \mathrm{H}_{26} \mathrm{~N}^{+}:$244.2060; found 244.2058 . 
Phenethylamine $5 \mathrm{~m}$
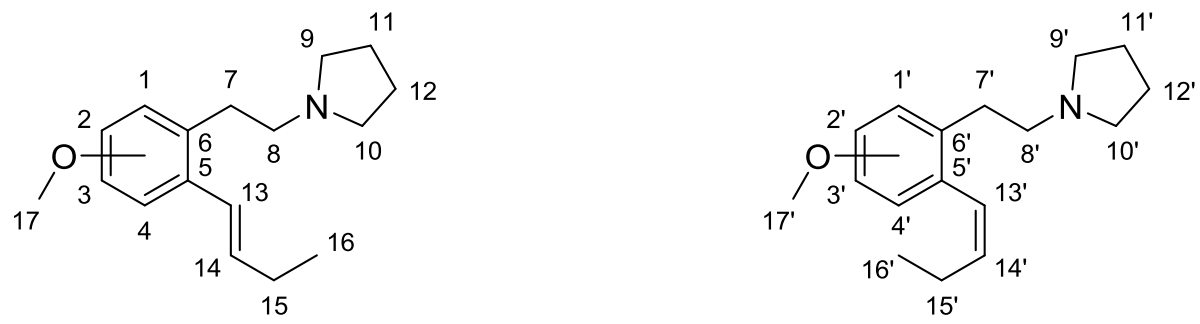

Starting materials:

6-Methoxyphthalazine (1f) (120 mg, $750 \mu \mathrm{mol}, 1.00$ equiv); BDLA (3.80 mg, $18.6 \mu \mathrm{mol}, 2.49 \mathrm{~mol} \%$ ); pyrrolidine (7a) $(74.7 \mu \mathrm{L}, 900 \mu \mathrm{mol}, 1.20$ equiv); butyraldehyde (6a) $(100 \mu \mathrm{L}, 1.09 \mathrm{mmol}, 1.45$ equiv); $\mathrm{NaBH}(\mathrm{OAc})_{3}$ (328 mg, $1.50 \mathrm{mmol}, 2.00$ equiv); $\mathrm{AcOH}$ (90.4 $\mu \mathrm{L}, 1.50 \mathrm{mmol}, 2.00$ equiv).

LED:

$448 \mathrm{~nm}$

Temperature:

$40{ }^{\circ} \mathrm{C}$

Purification:

Flash column chromatography ( 2 times) $\left(\mathrm{SiO}_{2}: 20 \mathrm{~g}\right.$, cyclohexane/EtOAc $\left.+5 \% \mathrm{NEt}_{3}, 10: 1\right)$.

Yield: mixture of $E / Z$-isomers $E-5 \mathrm{~m} / Z$-5m $(2.95: 1.00)$

and the corresponding constitutional isomers $\left(E-5 m^{\prime} / Z-5 m^{\prime}\right)$.

$142.4 \mathrm{mg}$ (549 $\mu \mathrm{mol}, 73 \%)$; pale yellow oil.

NMR spectra could not be assigned unambiguously

$E-5 m$ and $E-5 m^{\prime}:$

${ }^{1} \mathrm{H}$ NMR (400 MHz, $\mathrm{CDCl}_{3}$ with $0.03 \% \mathrm{v} / \mathrm{v}$ TMS): $\delta 7.35(\mathrm{~d}, J=8.2 \mathrm{~Hz}, 1 \mathrm{H}, \mathrm{H} 1$ or $\mathrm{H} 2$ or $\mathrm{H} 3$ or $\mathrm{H} 4), 7.07$ (dd, $J=10.4,8.8 \mathrm{~Hz}, 1 \mathrm{H}, \mathrm{H} 1$ or $\mathrm{H} 2$ or $\mathrm{H} 3$ or H4), $6.98-6.95(\mathrm{~m}, 2 \mathrm{H}, \mathrm{H} 1$ or H2 or H3 or H4), $6.79-6.68$ $(\mathrm{m}, 2 \mathrm{H}, \mathrm{H} 1$ or H2 or H3 or H4), 6.59 (dd, $J=19.4,15.6 \mathrm{~Hz}, 2 \mathrm{H}, \mathrm{H} 13), 6.14(\mathrm{dt}, J=15.5,6.6 \mathrm{~Hz}, 1 \mathrm{H}, \mathrm{H} 14)$, $6.03(\mathrm{dt}, J=15.5,6.6 \mathrm{~Hz}, 1 \mathrm{H}, \mathrm{H} 14), 3.81-3.77(\mathrm{~m}, 6 \mathrm{H}, \mathrm{H} 17), 2.91-2.73(\mathrm{~m}, 4 \mathrm{H}, \mathrm{H} 7), 2.66-2.54(\mathrm{~m}$, $12 \mathrm{H}, \mathrm{H} 8, \mathrm{H} 9, \mathrm{H} 10), 2.30-2.18(\mathrm{~m}, 4 \mathrm{H}, \mathrm{H} 15), 1.85-1.77(\mathrm{~m}, 8 \mathrm{H}, \mathrm{H} 11, \mathrm{H} 12), 1.09(\mathrm{td}, J=7.4,5.6 \mathrm{~Hz}, 6 \mathrm{H}$, H16).

${ }^{13} \mathrm{C}\left\{{ }^{1} \mathrm{H}\right\}$ NMR (101 MHz, CDCl 3 with $\left.0.03 \% \mathrm{v} / \mathrm{v} \mathrm{TMS}\right): \delta 158.6$ ( $\left.\mathrm{C}_{\mathrm{Ar}} \mathrm{OMe}\right), 158.3$ ( $\left.\mathrm{C}_{\mathrm{Ar}} \mathrm{OMMe}\right), 140.14$, $138.67,137.94,137.83,134.7$ (14), 132.8 (14), 130.87, 130.63, 130.33, 129.75, 129.72, 129.27, 127.13 (C4), 126.4 (C13), 125.8 (C13), 115.22, 115.13, 115.05, 112.82, 112.30, 112.06, 111.08, 110.99, 58.0 (C8), 57.6 (C8), 55.4 (C17), 55.3 (C17), 54.4 (C9, C10), 33.5 (C7), 32.5 (C7), 26.5 (C15), 26.4 (C15), 23.6 (C11, C12), 14.1 (C16), 13.9 (C16). 


\section{Z-5m and Z-5m':}

${ }^{1} \mathrm{H}$ NMR (400 MHz, $\mathrm{CDCl}_{3}$ with $\left.0.03 \% \mathrm{v} / \mathrm{v} \mathrm{TMS}\right): \delta 7.35$ (d, $J=8.2 \mathrm{~Hz}, 1 \mathrm{H}, \mathrm{H} 1^{\prime}$ or $\mathrm{H}^{\prime}$ ' or $\mathrm{H} 3^{\prime}$ or $\mathrm{H}^{\prime}$ ), 7.07 ( $\mathrm{dd}, J=10.4,8.8 \mathrm{~Hz}, 1 \mathrm{H}, \mathrm{H} 1^{\prime}$ or $\mathrm{H}^{\prime}$ or $\mathrm{H}^{\prime}$ or $\left.\mathrm{H}^{\prime}\right), 6.98-6.95\left(\mathrm{~m}, 2 \mathrm{H}, \mathrm{H} 1^{\prime}\right.$ or $\mathrm{H} 2^{\prime}$ or $\mathrm{H}^{\prime}$ or $\left.\mathrm{H}^{\prime}\right), 6.79-$ $6.68\left(\mathrm{~m}, 2 \mathrm{H}, \mathrm{H} 1^{\prime}\right.$ or $\mathrm{H}^{\prime}$ or $\mathrm{H}^{\prime}$ or $\left.\mathrm{H}^{\prime}\right), 6.44\left(\mathrm{dd}, J=17.2,11.4 \mathrm{~Hz}, 2 \mathrm{H}, \mathrm{H} 13^{\prime}\right), 5.74-5.60\left(\mathrm{~m}, 2 \mathrm{H}, \mathrm{H} 14^{\prime}\right)$, $3.81-3.77\left(\mathrm{~m}, 6 \mathrm{H}, \mathrm{H} 17^{\prime}\right), 2.91-2.73\left(\mathrm{~m}, 4 \mathrm{H}, \mathrm{H} 7^{\prime}\right), 2.66-2.54\left(\mathrm{~m}, 12 \mathrm{H}, \mathrm{H} 8^{\prime}, \mathrm{H} 9^{\prime}, \mathrm{H} 10^{\prime}\right), 2.14(\mathrm{pd}, J=$ 7.4, $\left.1.5 \mathrm{~Hz}, 4 \mathrm{H}, \mathrm{H} 15^{\prime}\right), 1.85-1.77$ (m, 8H, H11', H12'), 0.99 (td, J = 7.5, 4.4 Hz, 6H, H16').

${ }^{13} \mathrm{C}\left\{{ }^{1} \mathrm{H}\right\}$ NMR (101 MHz, $\mathrm{CDCl}_{3}$ with 0.03\% v/v TMS): $\delta 158.7$ ( $\left.{ }^{\prime}{ }_{\mathrm{Ar}}-\mathrm{OMe}\right), 140.14,138.67,137.94,137.83$, 135.3 (C14'), 134.6 (C14'), 130.87, 130.63, 130.33, 129.75, 129.72, 129.27, 127.1 (C13'), 126.6 (C13'), $115.22,115.13,115.05,112.82,112.30,112.06,111.08,110.99,57.7$ (C8'), 57.3 (C8'), 55.33 (C17'),

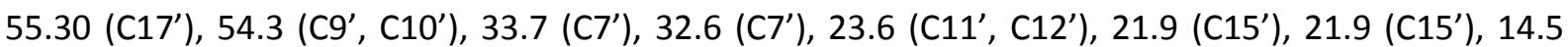
(C16'), $14.4\left(\mathrm{C}^{\prime} 6^{\prime}\right)$.

HRMS (ESI) m/z [M+H] calc. for $\mathrm{C}_{17} \mathrm{H}_{26} \mathrm{NO}^{+}$: 260.2009; found: 260.2012 . 


\section{Phenethylenamine 4a}
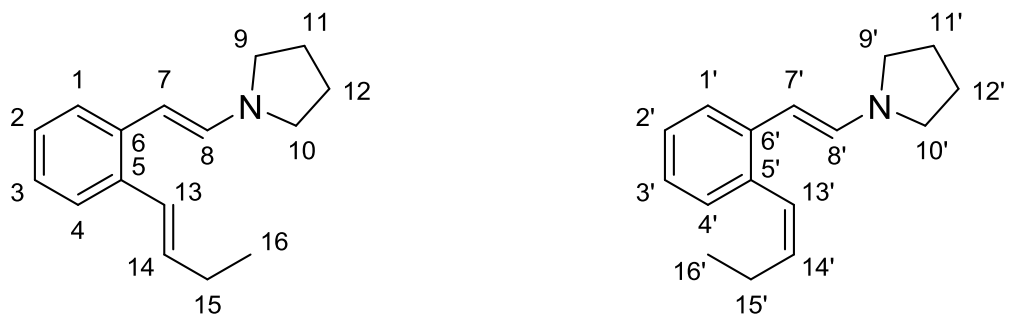

The reaction was set up in a nitrogen filled glovebox.

Phthalazine (1a) (105 mg, $795 \mu \mathrm{mol}, 1.00$ equiv) and BDLA (4.00 mg, $19.6 \mu \mathrm{mol}, 2.47 \mathrm{~mol} \%$ ) were suspended in THF (dry, degassed) $(2.00 \mathrm{~mL}$ ). Then pyrrolidine (7a) $(79.6 \mu \mathrm{L}, 960 \mu \mathrm{mol}, 1.21$ equiv) was added (yellow suspension turned colorless). The reaction vessel was sealed and the reaming steps of the reaction were carried out in a fume hood. The reaction mixture was irradiated irradiated by a $425-430 \mathrm{~nm}$ LED and butyraldehyde (6a) $(110 \mu \mathrm{L}, 1.20 \mathrm{mmol}, 1.50$ equiv) was added dropwise. Gas evolution started almost immediately. The yellow solution was stirred at $\mathrm{rt}$ for $15 \mathrm{~h}$. Afterwards all volatile compounds were removed under vacuum ( $\mathrm{rt},<1 \mathrm{mbar}$, recondensed into a liquid $\mathrm{N}_{2}$ cooling trap). The remaining orange solution was purified by distillation (Kugelrohr-Apperatus). A pale yellow oil was obtained $\left(150^{\circ} \mathrm{C}\right.$ at $\left.4 \times 10^{-2} \mathrm{mbar}\right)$.

Yield: mixture of $E / Z$-isomers $E-4 a / Z-4 a(1.00: 2.60)$

$95.2 \mathrm{mg}$ (418 $\mu \mathrm{mol}, 54 \%)$; pale yellow oil.

\section{E-4a:}

${ }^{1} \mathrm{H}$ NMR (400 MHz, $\mathrm{CDCl}_{3}$ with $\left.0.03 \% \mathrm{v} / \mathrm{v} \mathrm{TMS}\right): \delta 7.35-7.29(\mathrm{~m}, 1 \mathrm{H}, \mathrm{H} 2), 7.25$ (d, $\left.J=6.4 \mathrm{~Hz}, 1 \mathrm{H}, \mathrm{H} 1\right)$, $7.14-7.06(\mathrm{~m}, 1 \mathrm{H}, \mathrm{H} 4), 7.00-6.91(\mathrm{~m}, 1 \mathrm{H}, \mathrm{H} 3), 6.92(\mathrm{~d}, J=13.7 \mathrm{~Hz}, 1 \mathrm{H}, \mathrm{H8}), 6.67(\mathrm{~d}, J=15.7 \mathrm{~Hz}, 1 \mathrm{H}$, $\mathrm{H} 13), 6.09$ (dt, $J=15.6,6.5 \mathrm{~Hz}, 1 \mathrm{H}, \mathrm{H} 14), 5.24(\mathrm{~d}, J=13.7 \mathrm{~Hz}, 1 \mathrm{H}, \mathrm{H} 7), 3.29-3.19(\mathrm{~m}, 4 \mathrm{H}, \mathrm{H} 9, \mathrm{H} 10), 2.31$ $-2.22(\mathrm{~m}, 2 \mathrm{H}, \mathrm{H} 15), 1.96-1.90(\mathrm{~m}, 4 \mathrm{H}, \mathrm{H} 11, \mathrm{H} 12), 1.12(\mathrm{t}, J=7.5 \mathrm{~Hz}, 3 \mathrm{H}, \mathrm{H} 16)$.

${ }^{13} \mathrm{C}\left\{{ }^{1} \mathrm{H}\right\}$ NMR (101 MHz, $\mathrm{CDCl}_{3}$ with 0.03\% v/v TMS): $\delta 137.5$ (C6), 137.1 (C8), 133.9 (C5), 133.3 (C14), 127.7 (C13), 127.0 (C4), 126.6 (C2), 123.5 (C1), 123.5 (C3), 95.0 (C7), 49.1 (C9, C10), 26.5 (C15), 25.4 (C11, C12), 14.1 (C16).

\section{Z-4a:}

${ }^{1} \mathrm{H}$ NMR (400 MHz, $\mathrm{CDCl}_{3}$ with $\left.0.03 \% \mathrm{v} / \mathrm{v} \mathrm{TMS}\right): \delta 7.35-7.29\left(\mathrm{~m}, 1 \mathrm{H}, \mathrm{H} 1^{\prime}\right), 7.14-7.06\left(\mathrm{~m}, 2 \mathrm{H}, \mathrm{H2}{ }^{\prime}, \mathrm{H} 4^{\prime}\right)$, $7.00\left(\mathrm{~d}, J=13.8 \mathrm{~Hz}, 1 \mathrm{H}, \mathrm{H} 8^{\prime}\right), 7.00-6.91\left(\mathrm{~m}, 1 \mathrm{H}, \mathrm{H} 3^{\prime}\right), 6.43\left(\mathrm{~d}, J=11.4 \mathrm{~Hz}, 1 \mathrm{H}, \mathrm{H} 13^{\prime}\right), 5.67(\mathrm{dt}, J=11.4$, $7.4 \mathrm{~Hz}, 1 \mathrm{H}, \mathrm{H} 14^{\prime}$ ), 5.14 (d, J = $13.8 \mathrm{~Hz}, 1 \mathrm{H}, \mathrm{H}^{\prime}$ ), $3.29-3.19$ (m, 4H, H9', H10'), 2.19 (qd, J = 7.4, $1.7 \mathrm{~Hz}$, $\left.2 \mathrm{H}, \mathrm{H} 15^{\prime}\right), 1.96-1.90\left(\mathrm{~m}, 4 \mathrm{H}, \mathrm{H} 11^{\prime}, \mathrm{H} 12^{\prime}\right), 1.02\left(\mathrm{t}, \mathrm{J}=7.5 \mathrm{~Hz}, 3 \mathrm{H}, \mathrm{H} 16^{\prime}\right)$.

${ }^{13} \mathrm{C}\left\{{ }^{1} \mathrm{H}\right\}$ NMR (101 MHz, CDCl 3 with $0.03 \%$ v/v TMS): $\delta 138.3$ (C6'), 136.6 (C8'), 133.9 (C14'), 133.1 (C5'), 129.7 (C4'), 128.2 (C13'), 127.0 (C2'), 122.5 (C3'), 122.3 (C1'), 95.4 (C7'), 49.1 (C9', C10'), 25.4 (C11', C12'), 22.0 (C15'), 14.6 (C16').

HRMS (ESI) m/z [M+H] calc. for $\mathrm{C}_{16} \mathrm{H}_{22} \mathrm{~N}^{+}$: 228.1747; found: 228.1751 . 


\section{Optimization of Irradiation Wavelength for the Domino IEDDA/PIRO Reaction:}

The reaction was set up in a nitrogen filled glovebox.

Phthalazine (1a) (49.3 mg, $375 \mu \mathrm{mol}, 1.00$ equiv), 1,3,5-trimethoxybenzene (TMB) (21.0 mg, $124 \mu \mathrm{mol}$, 0.333 equiv), and BDLA ( $1.90 \mathrm{mg}, 9.32 \mu \mathrm{mol}, 2.49$ equivl) were suspended in THF- $\mathrm{d}_{8}$ (dry, degassed) $(1.00 \mathrm{~mL})$. Then, pyrrolidine ( $7 \mathrm{a})(37.3 \mu \mathrm{L}, 450 \mu \mathrm{mol}, 1.20$ equiv) was added, the reaction vessel was sealed and the reaction was continued in a fume hood. The reaction mixture was heated to $30{ }^{\circ} \mathrm{C}$ and irradiated with a LED. Then butyraldehyde (6a) $(50.0 \mu \mathrm{L}, 544 \mu \mathrm{mol}, 1.45$ equiv) was added. Gas evolution was observed after a few minutes. The reaction was kept irradiated overnight. Afterwards the vessel was sealed, put back into the glovebox and a NMR sample was prepared.
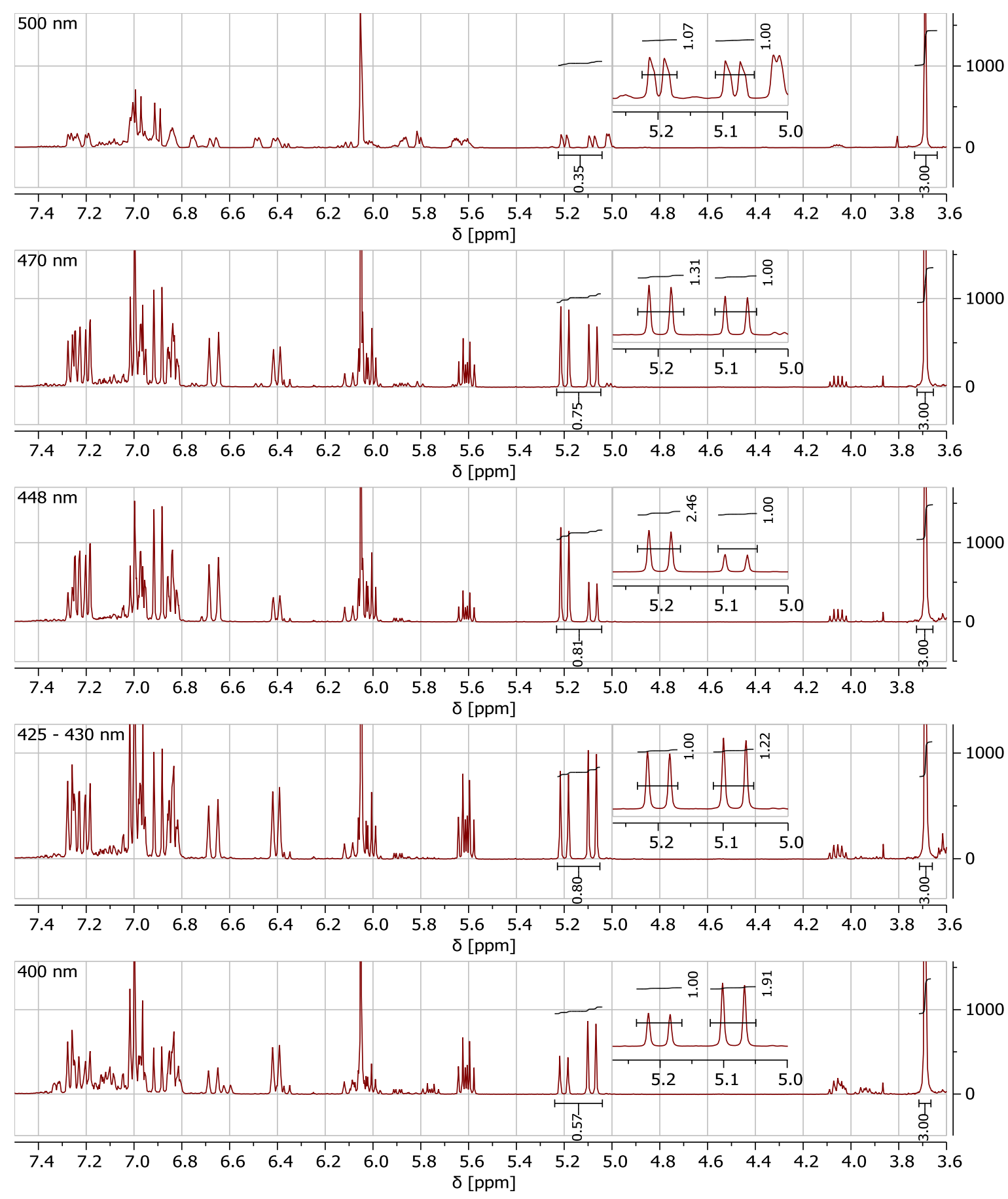


\section{Isomerization Monitoring of IEDDA/PIRO Product:}

The reaction was set up in a nitrogen filled glovebox.

Phthalazine (1a) (148 mg, $1.13 \mathrm{mmol}, 1.00$ equiv), 1,3,5-trimethoxybenzene (TMB) $(63.0 \mathrm{mg}$, $371 \mu \mathrm{mol}, 0.333$ equiv), and BDLA $(5.70 \mathrm{mg}, 28.0 \mu \mathrm{mol}, 2.49 \mathrm{~mol} \%)$ were suspended in THF- $\mathrm{d}_{8}$ (dry, degassed) (3.00 mL). Then, pyrrolidine (7a) $(112 \mu \mathrm{L}, 1.35 \mathrm{mmol}, 1.20$ equiv) was added, the reaction vessel was sealed and the reaction was continued in a fume hood. The reaction mixture was heated to $30{ }^{\circ} \mathrm{C}$ and irradiated with a $425-430 \mathrm{~nm}$ LED. Then butyraldehyde (6a) $(150 \mu \mathrm{L}, 1.63 \mathrm{mmol}, 1.45$ equiv) was added. Gas evolution was observed after a few minutes. Aliquots $(0.70 \mathrm{~mL})$ were taken in intervals ( $2 \mathrm{~h}, 4 \mathrm{~h}, 10 \mathrm{~h}$ ) under Schlenk conditions.
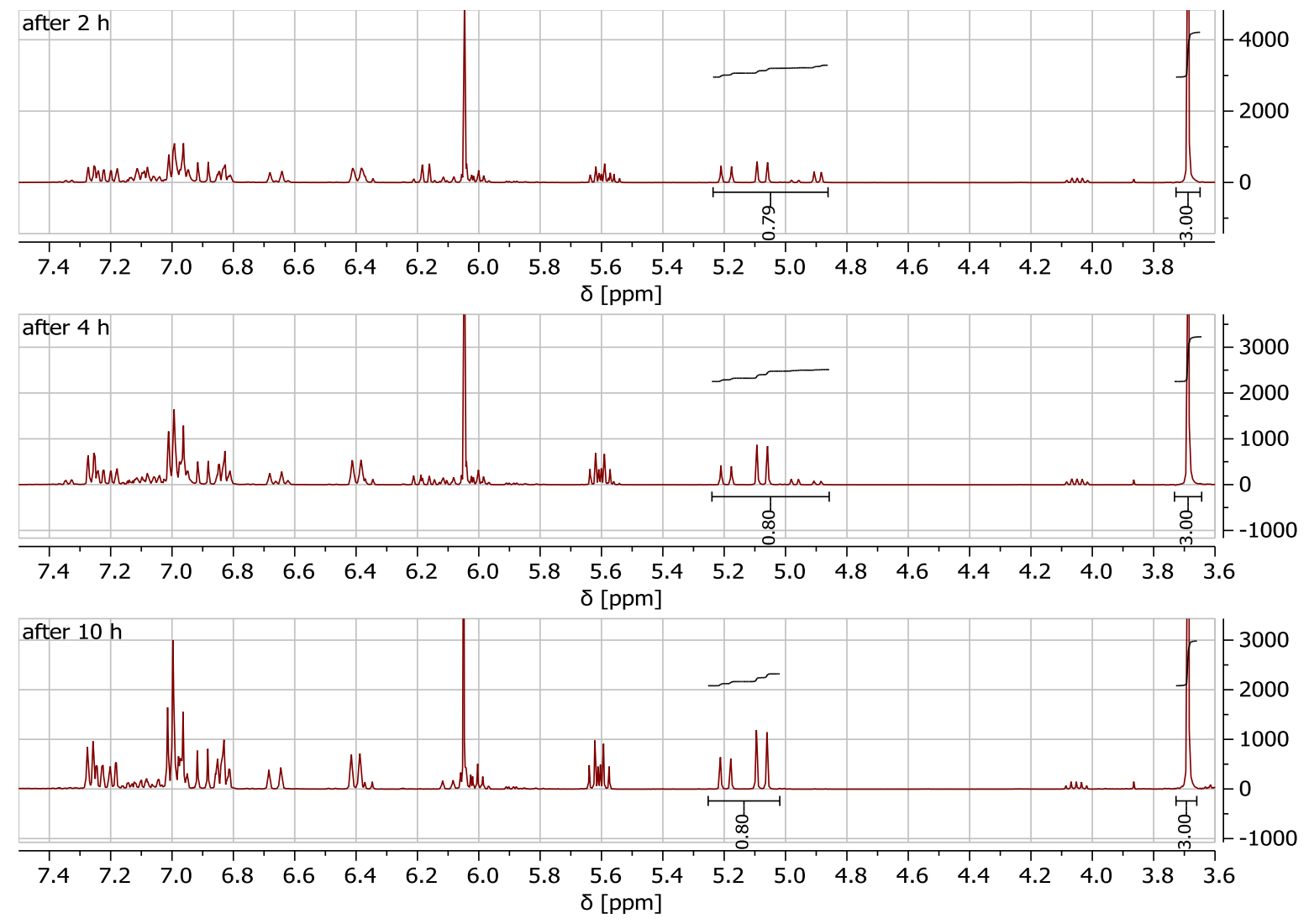


\section{Degradation of BDLA-Phthalazine Complex by Irradiation:}

UV/Vis Spectrum of BDLA-Phthalazine Complex

Sample was prepared in a nitrogen filled glovebox.

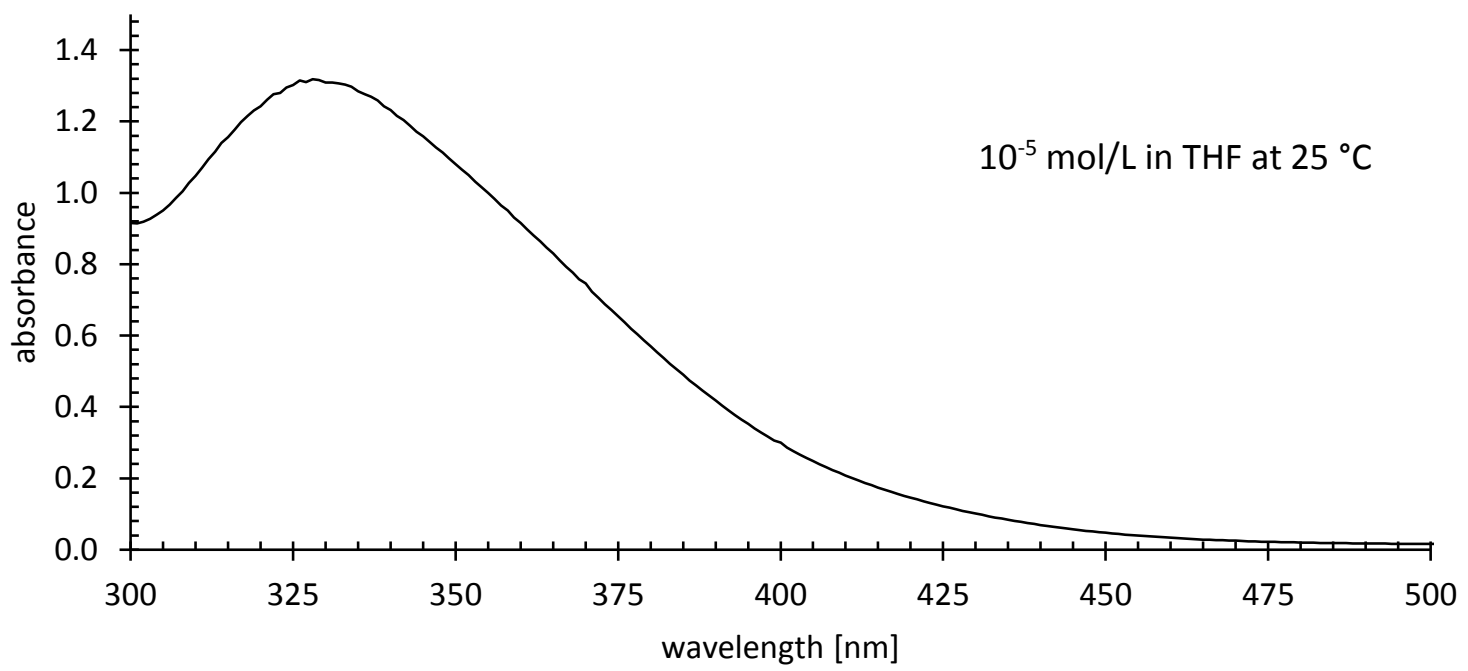

NMR analysis of BDLA-Phthalazine Complex under irradiation.

Reaction was set up in a nitrogen filled glovebox.

Phthalazine (1a) $(3.30 \mathrm{mg}, 25.1 \mu \mathrm{mol}, 1.00$ equiv) and BDLA (5.10 mg, $25.0 \mu \mathrm{mol}, 1.00$ equiv) were suspended in THF- $\mathrm{d}_{8}$ (dry, degassed) $(1.00 \mathrm{~mL})$ in a screw cap NMR tube, resulting in a saturated solution. A ${ }^{1} \mathrm{H}$ NMR was measured before it was irradiated by a $425-430 \mathrm{~nm}$ LED for $15 \mathrm{~h}$ at $30^{\circ} \mathrm{C}$. Afterwards a second ${ }^{1} \mathrm{H}$ NMR was measured.

$1 \mathrm{H}$ NMR $(400 \mathrm{MHz}$, THF-D8)

before irradiation
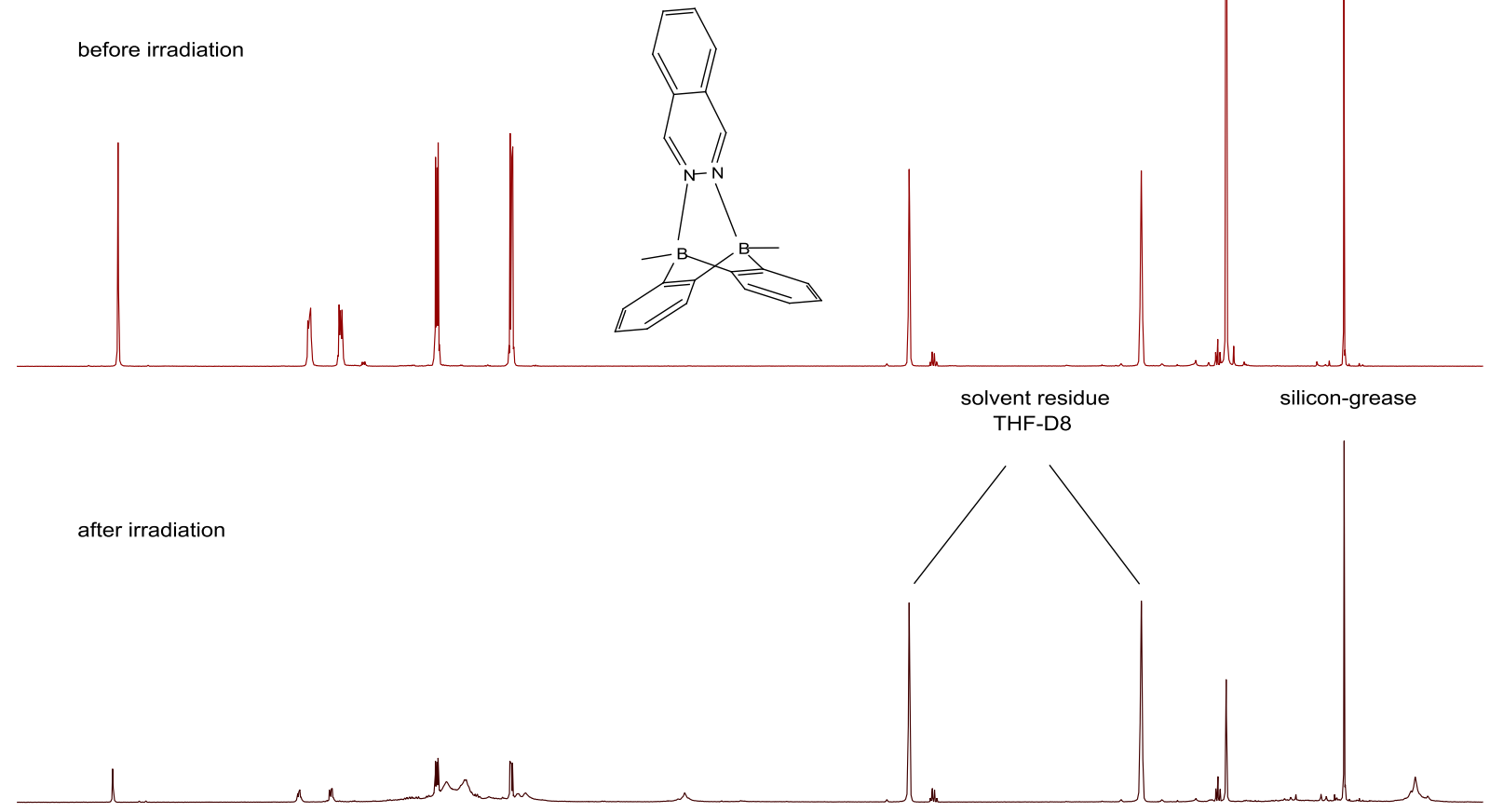

$\begin{array}{llllllllllllllllllllllllll}10.5 & 10.0 & 9.5 & 9.0 & 8.5 & 8.0 & 7.5 & 7.0 & 6.5 & 6.0 & 5.5 & 5.0 & 4.5 & 4.0 & 3.5 & 3.0 & 2.5 & 2.0 & 1.5 & 1.0 & 0.5 & 0.0 & -0.5 & -1\end{array}$ 
NMR Spectra:

${ }^{1} \mathrm{H}$ NMR of $5 a$

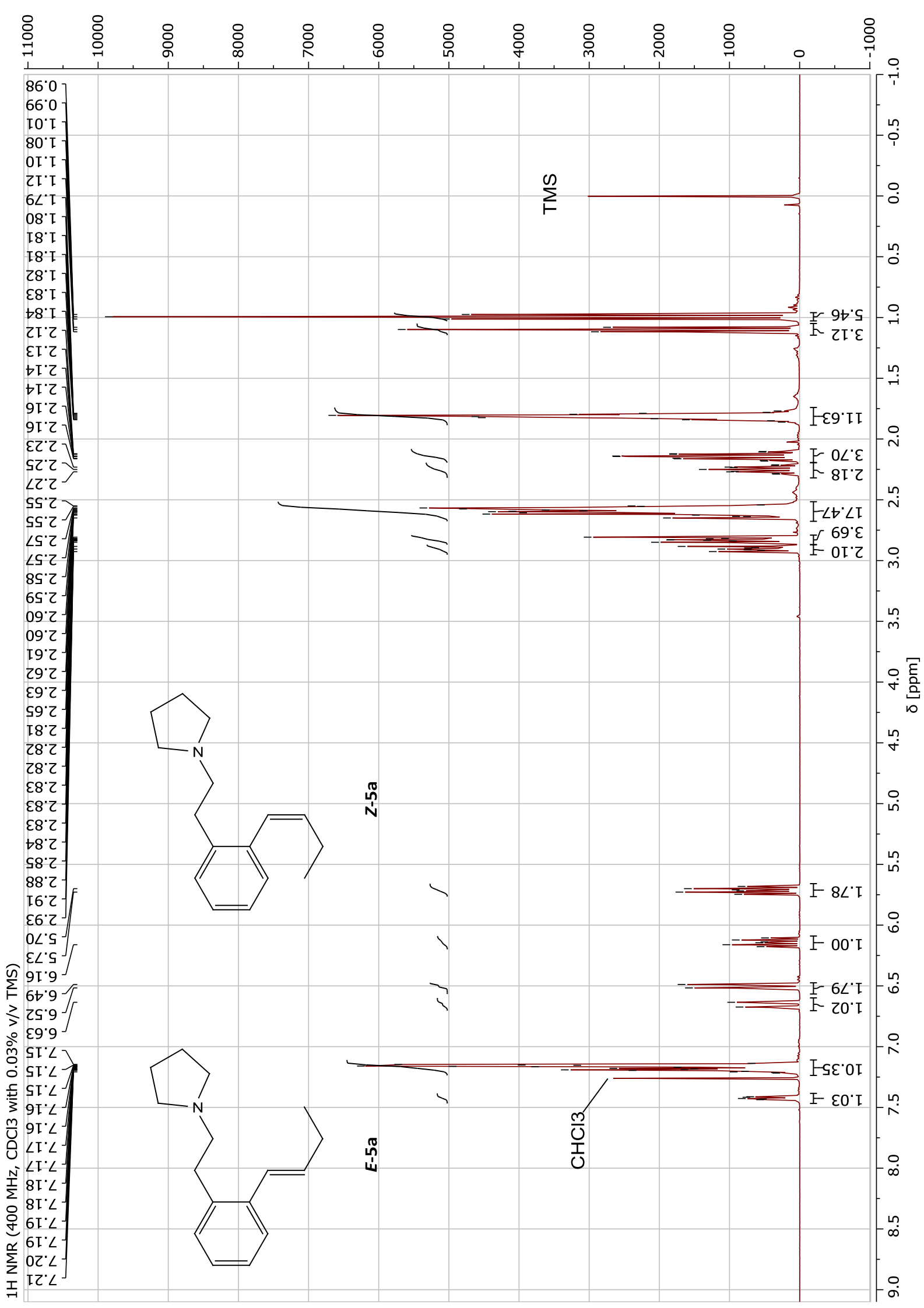


${ }^{13} \mathrm{C}\left\{{ }^{1} \mathrm{H}\right\}$ NMR of $5 a$

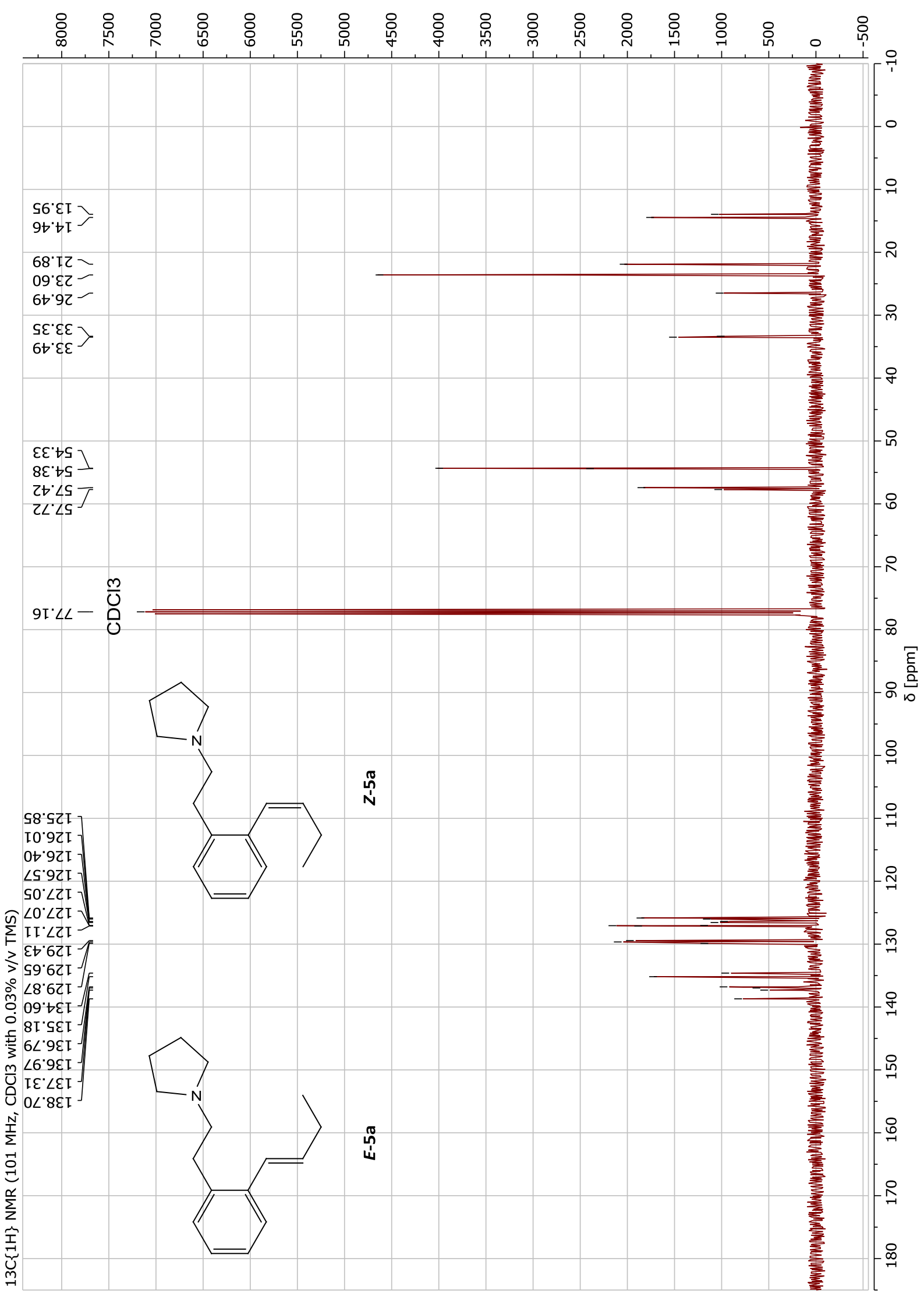


${ }^{1} \mathrm{H}$ NMR of $5 b$

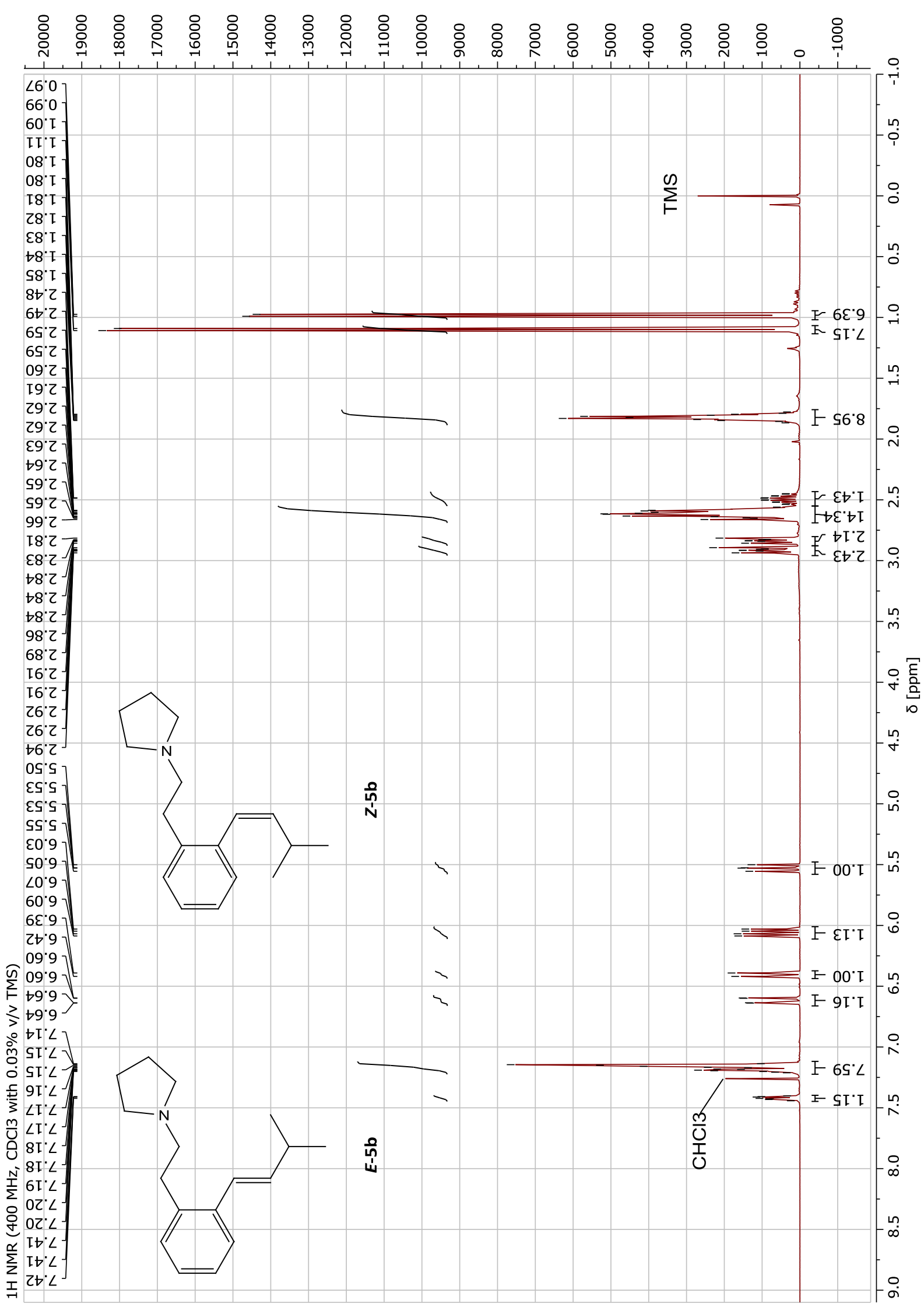


${ }^{13} \mathrm{C}\left\{{ }^{1} \mathrm{H}\right\}$ NMR of $5 b$

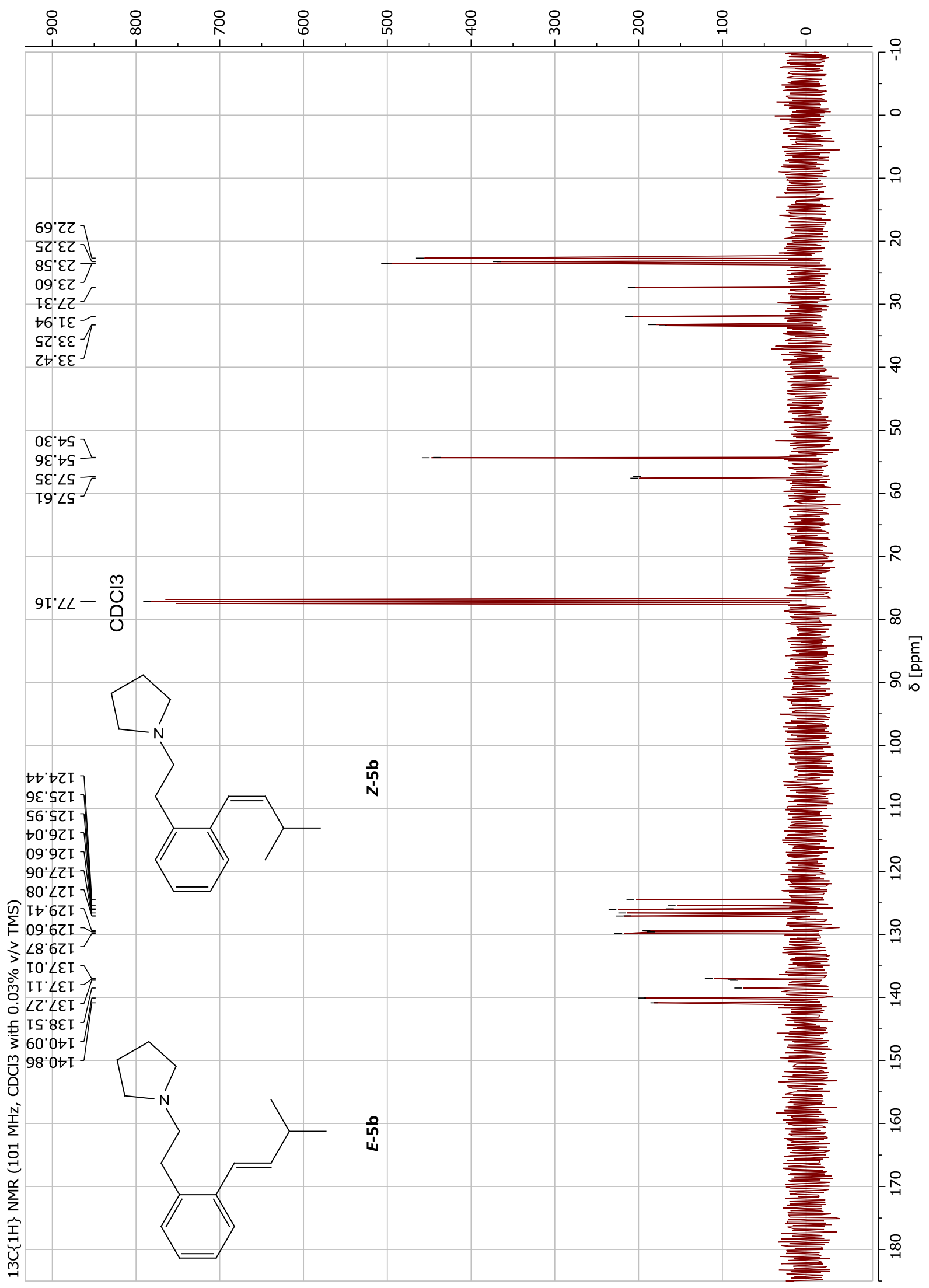

29 
${ }^{1} \mathrm{H}$ NMR of $5 c$

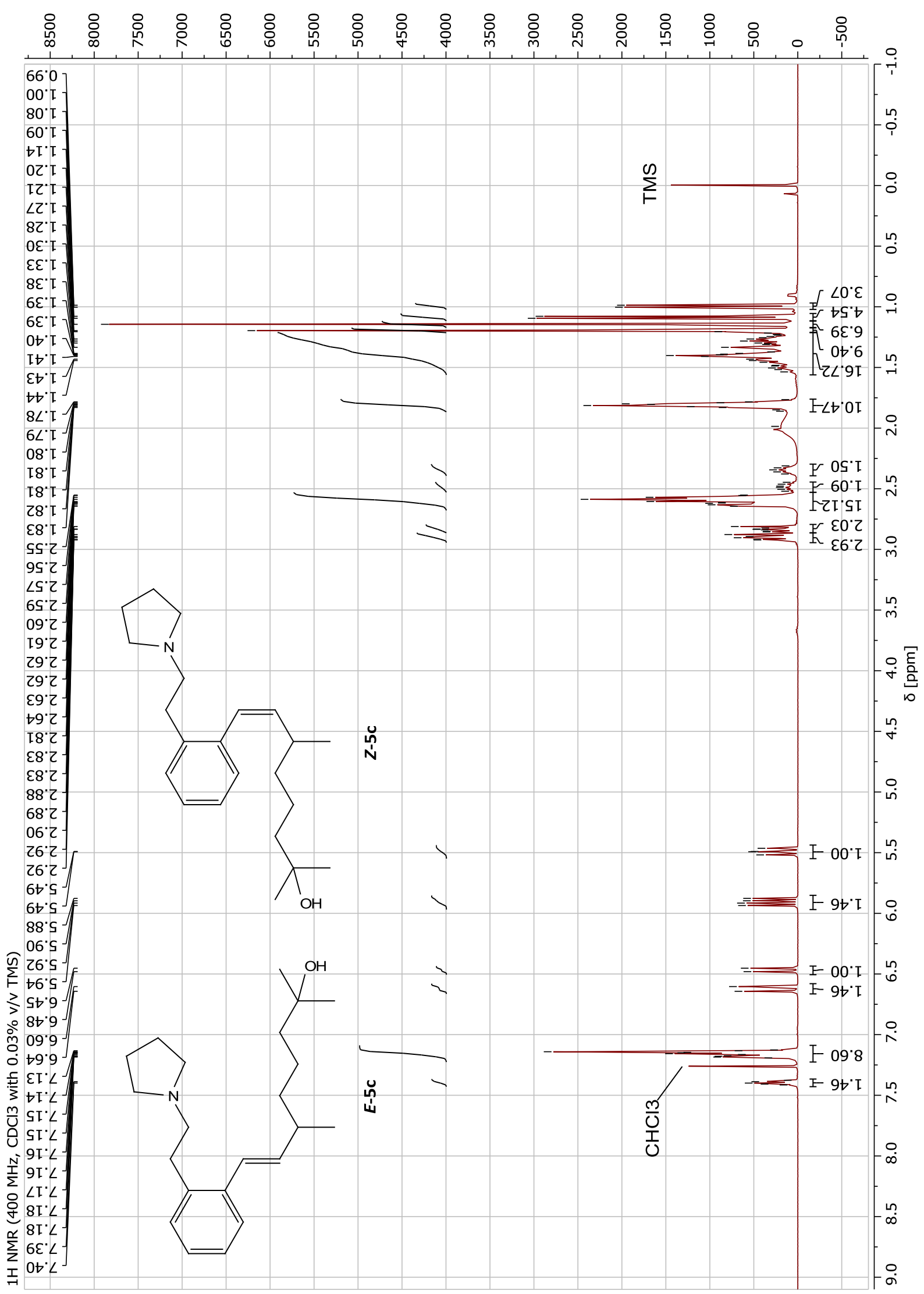


${ }^{13} \mathrm{C}\left\{{ }^{1} \mathrm{H}\right\}$ NMR of $5 \mathrm{C}$
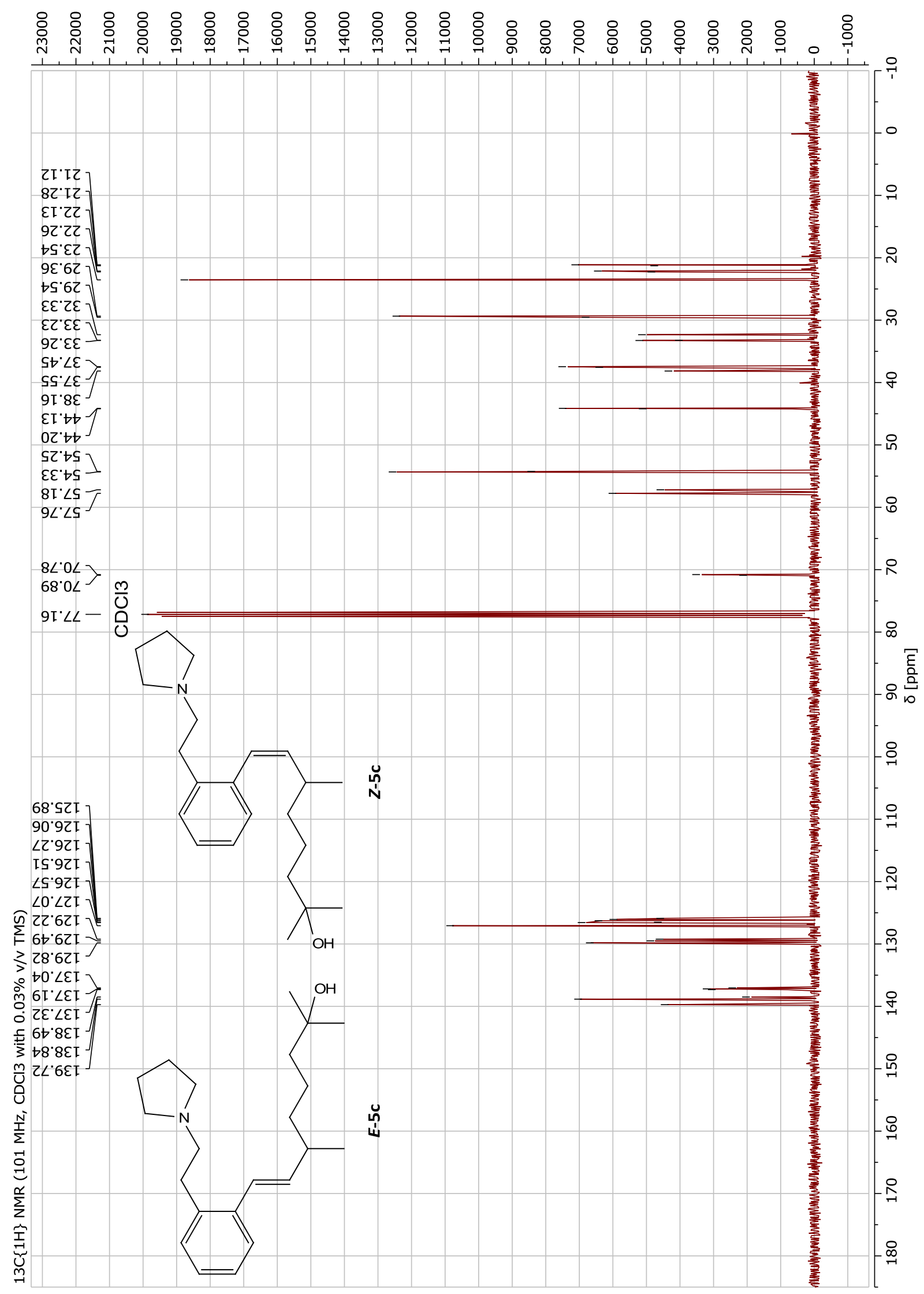
${ }^{1} \mathrm{H}$ NMR of $5 d$

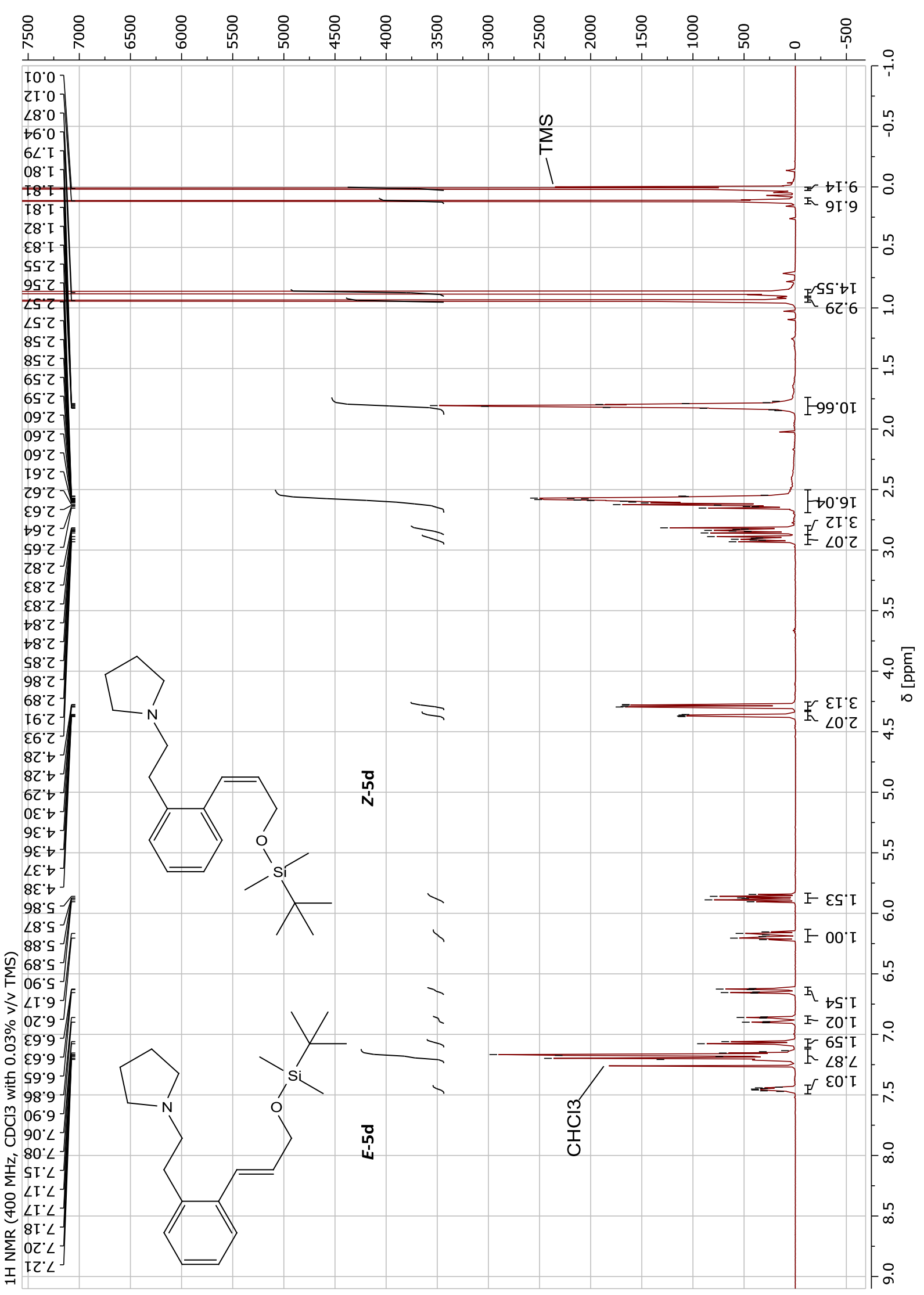


${ }^{13} \mathrm{C}\left\{{ }^{1} \mathrm{H}\right\}$ NMR of $5 \mathrm{~d}$

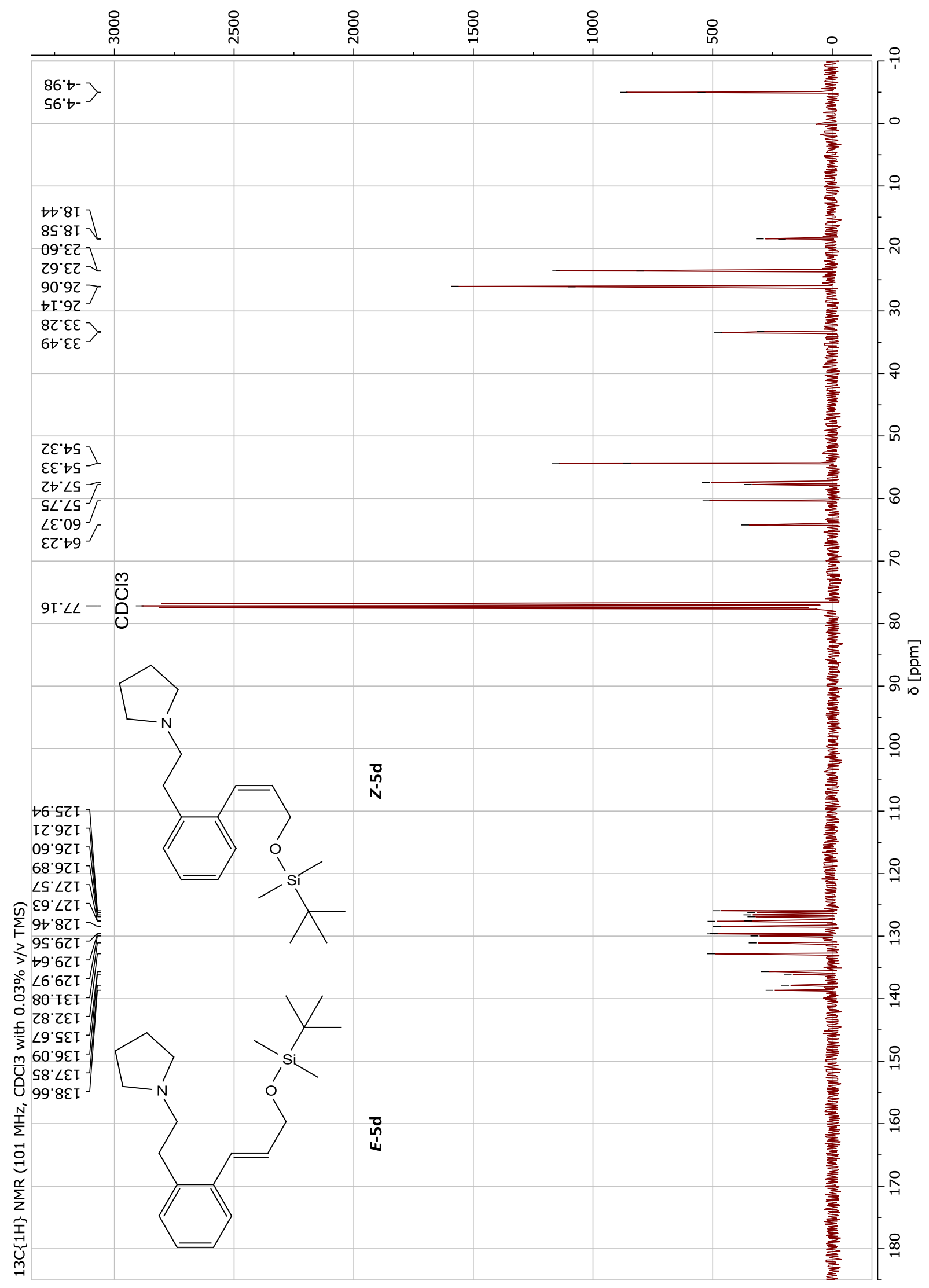


${ }^{1} \mathrm{H}$ NMR of $5 e$

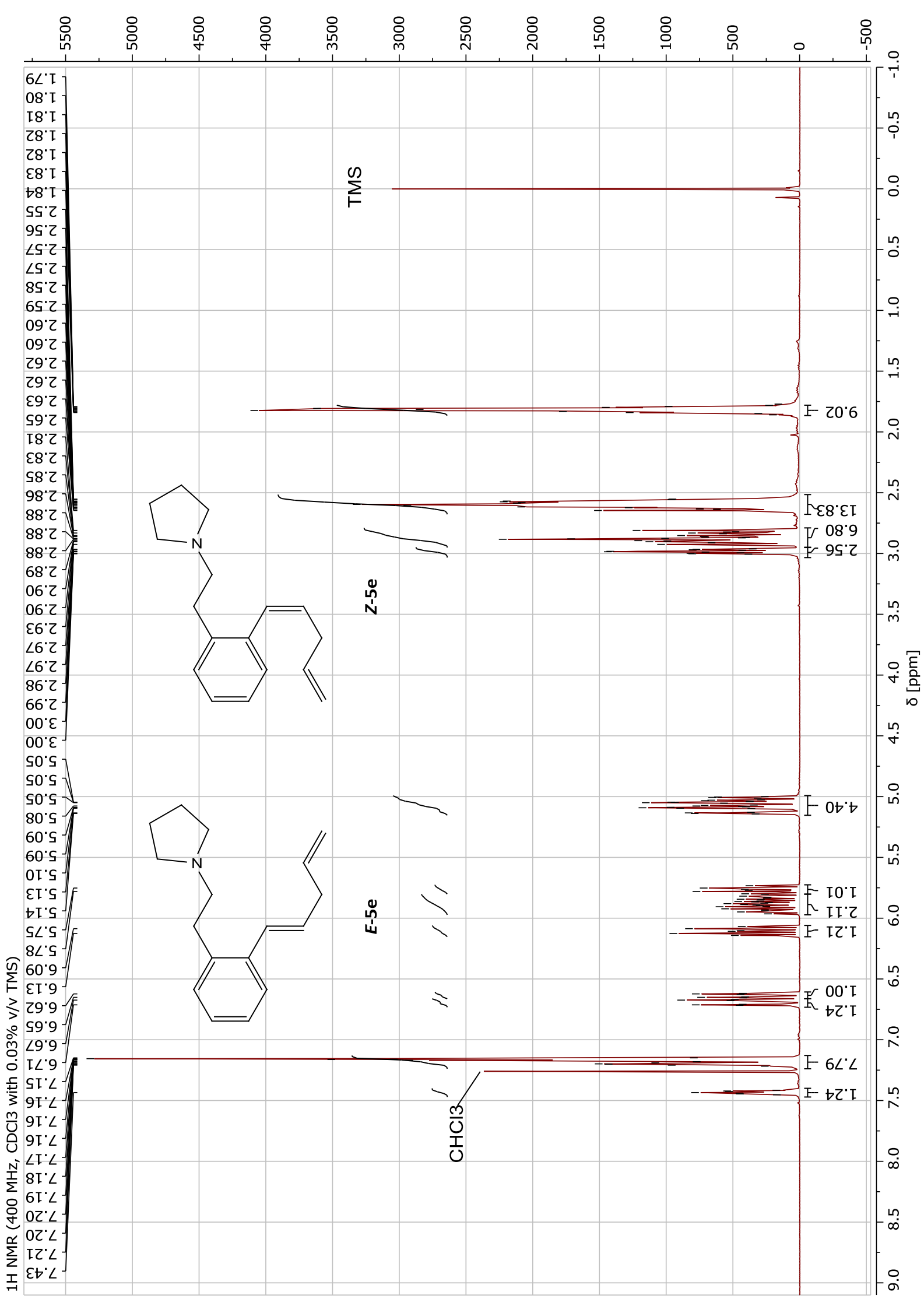


${ }^{13} \mathrm{C}\left\{{ }^{1} \mathrm{H}\right\}$ NMR of $5 e$

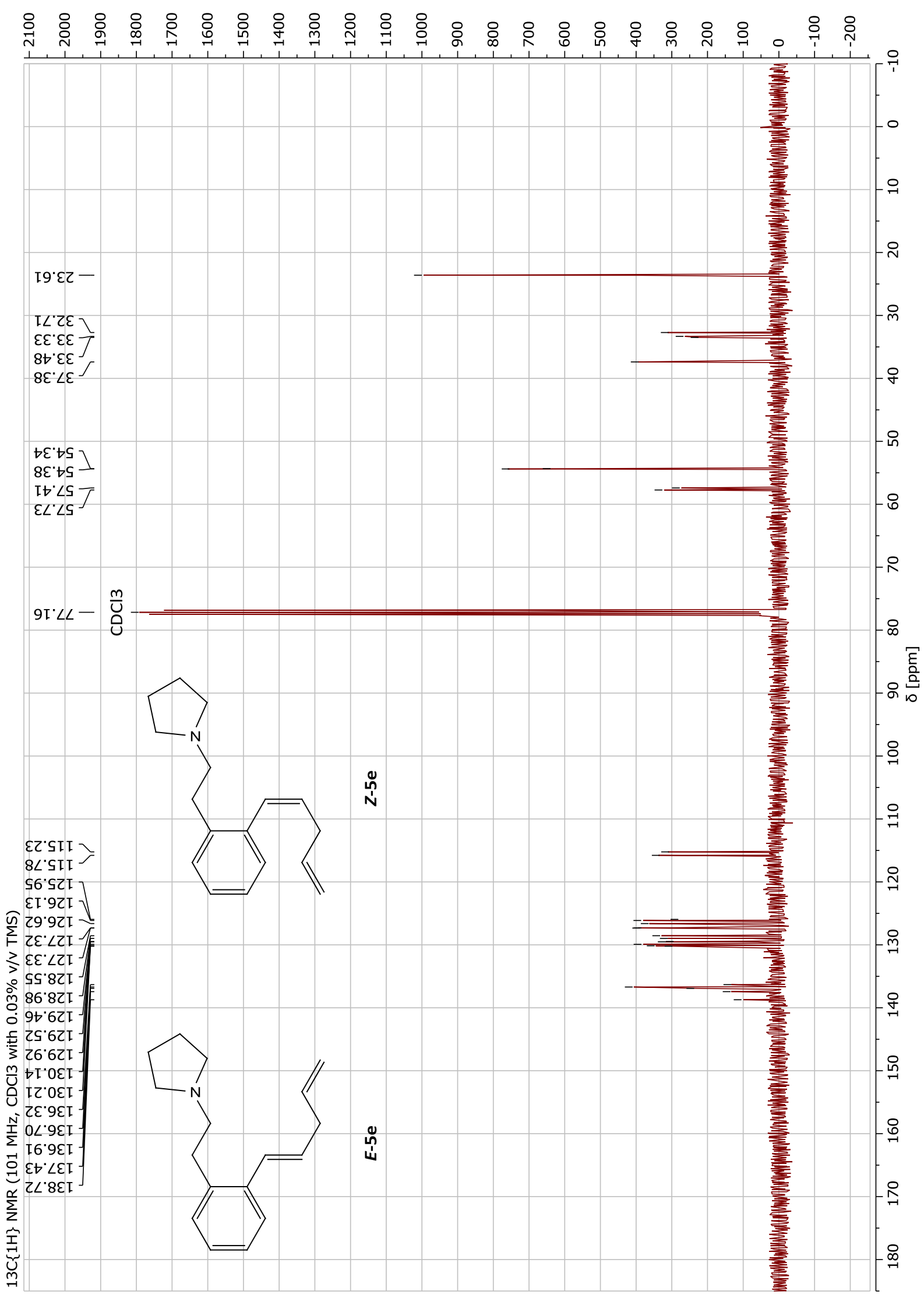


${ }^{1} \mathrm{H}$ NMR of $5 f$

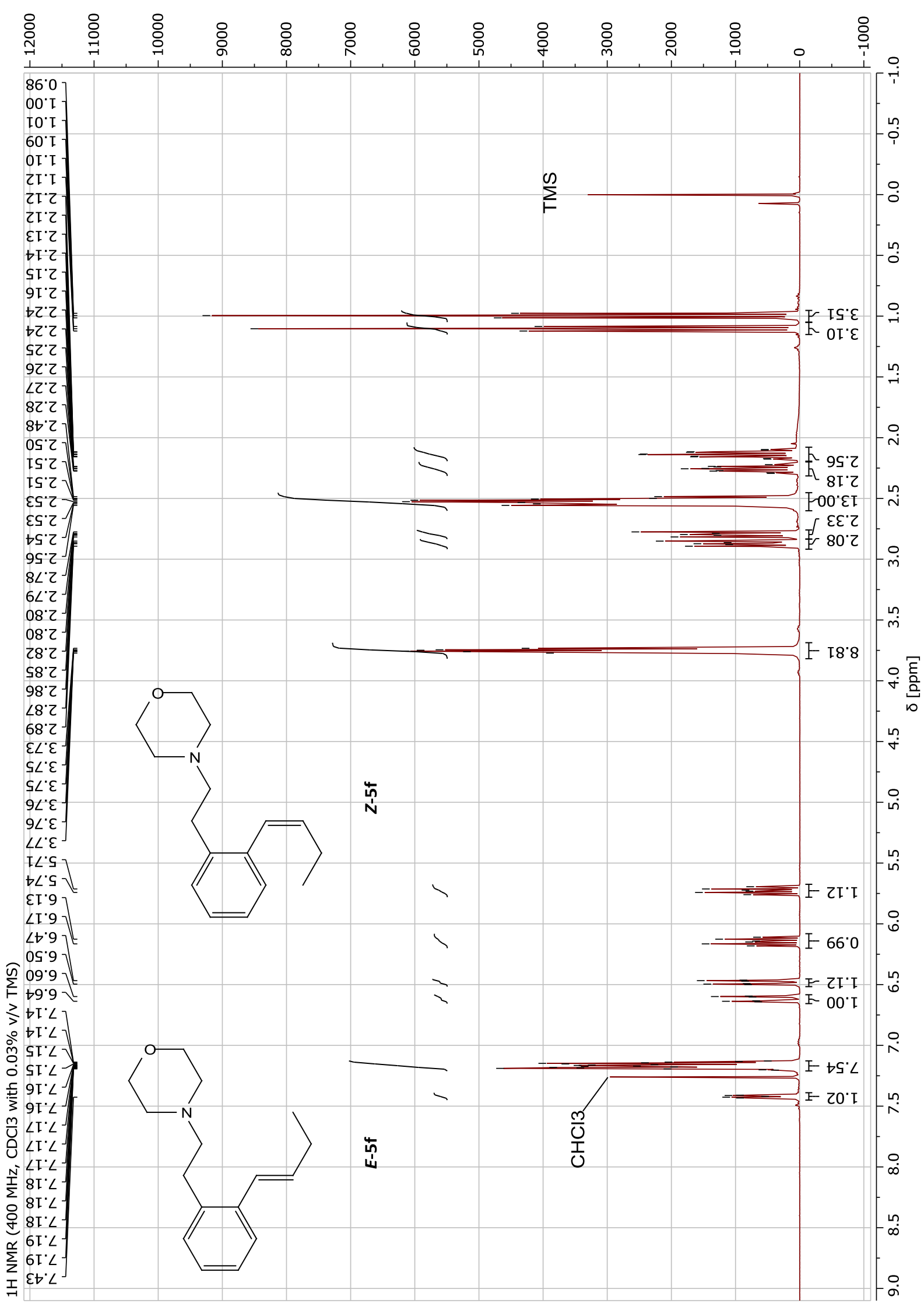


${ }^{13} \mathrm{C}\left\{{ }^{1} \mathrm{H}\right\}$ NMR of $5 f$

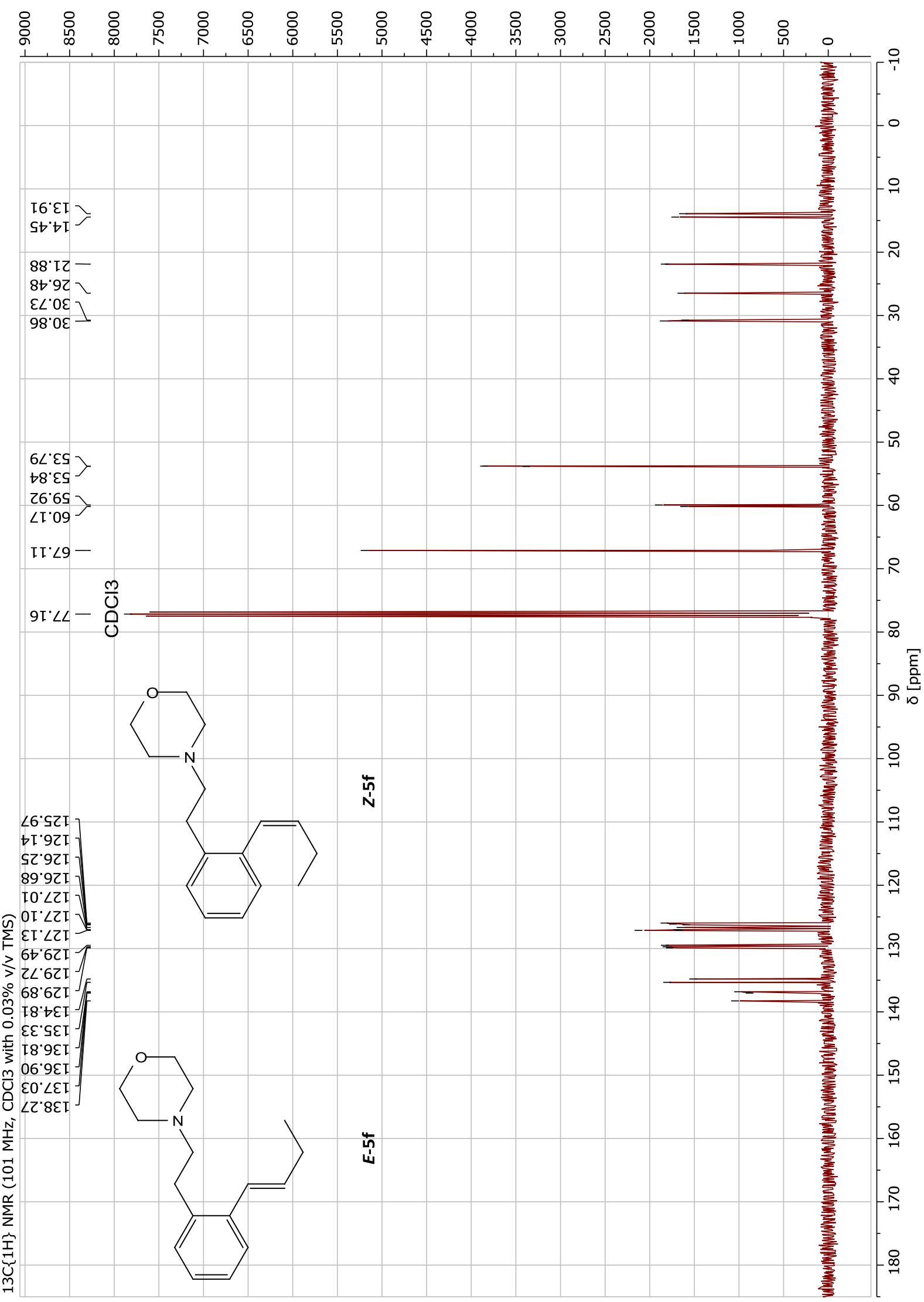


${ }^{1} \mathrm{H}$ NMR of $5 g$

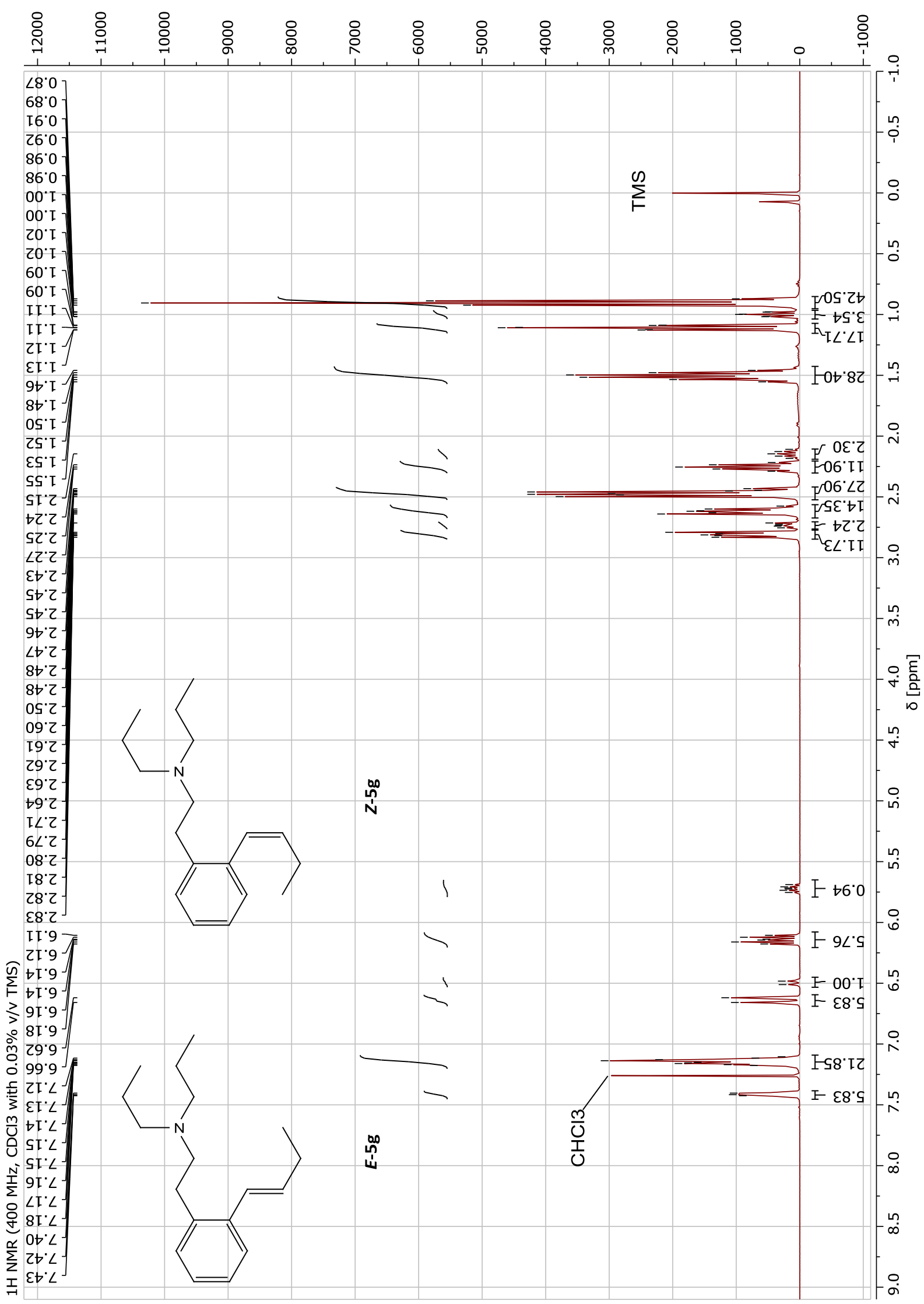


${ }^{13} \mathrm{C}\left\{{ }^{1} \mathrm{H}\right\}$ NMR of $5 \mathrm{~g}$

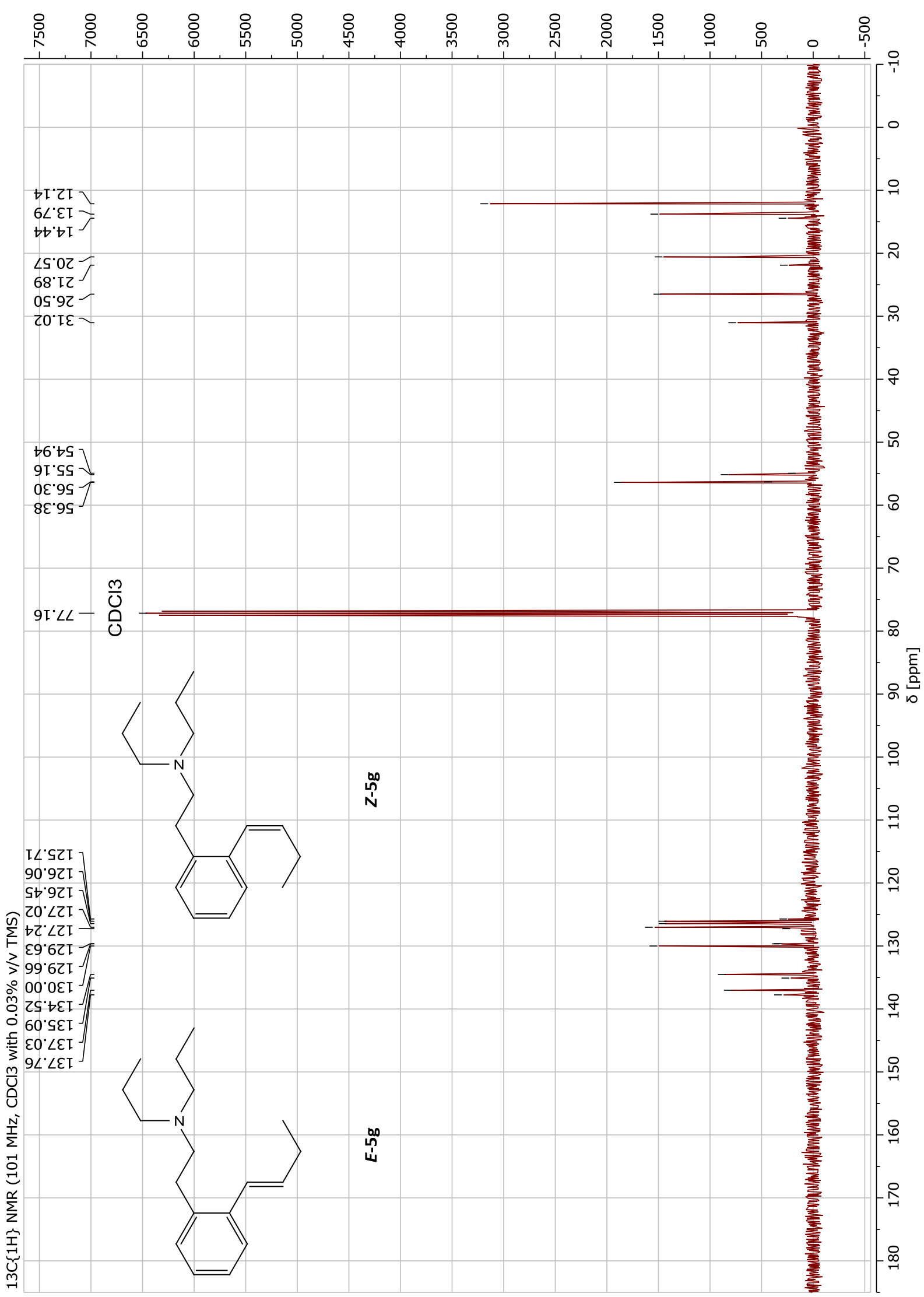


${ }^{1} \mathrm{H}$ NMR of $5 \mathrm{~h}$

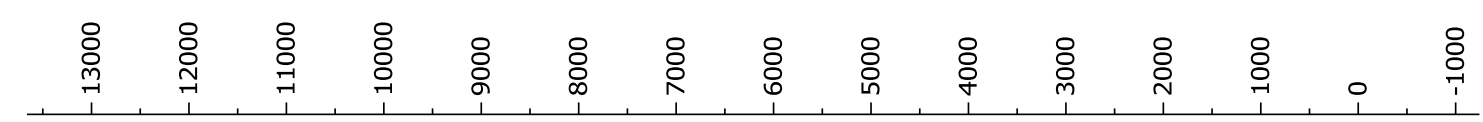

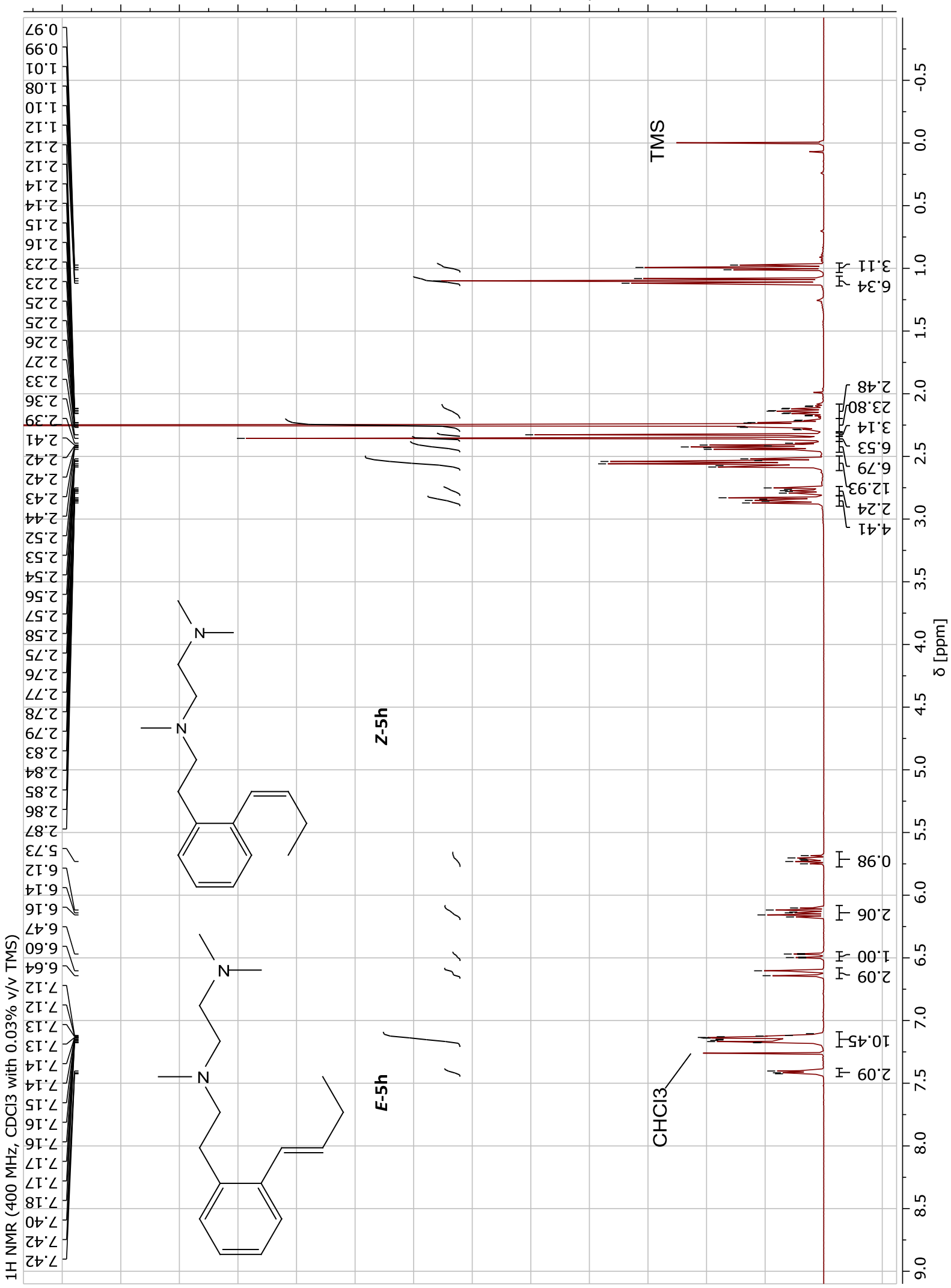


${ }^{13} \mathrm{C}\left\{{ }^{1} \mathrm{H}\right\}$ NMR of $5 \mathrm{~h}$

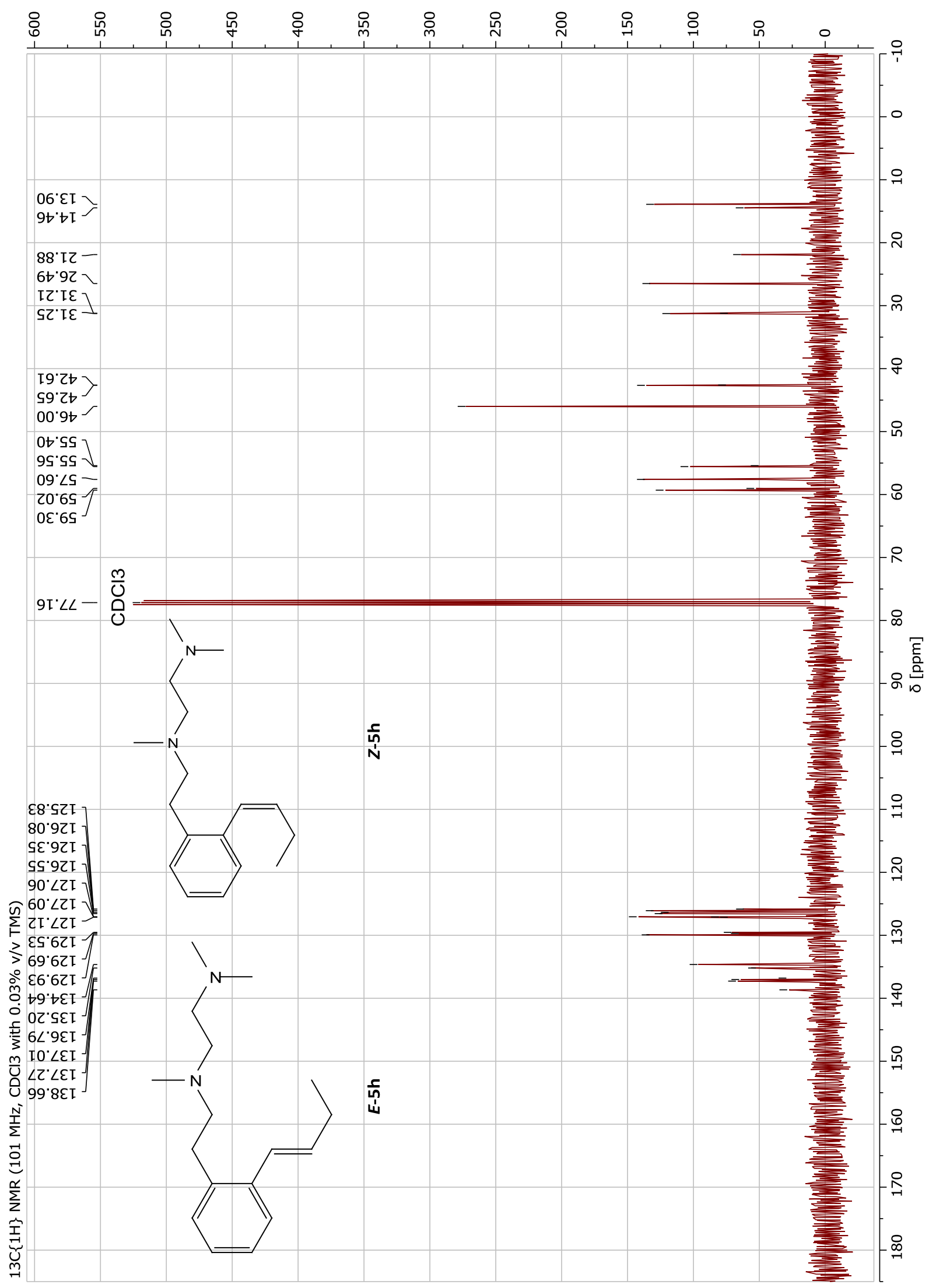

41 
${ }^{1} \mathbf{H}$ NMR of $5 i$

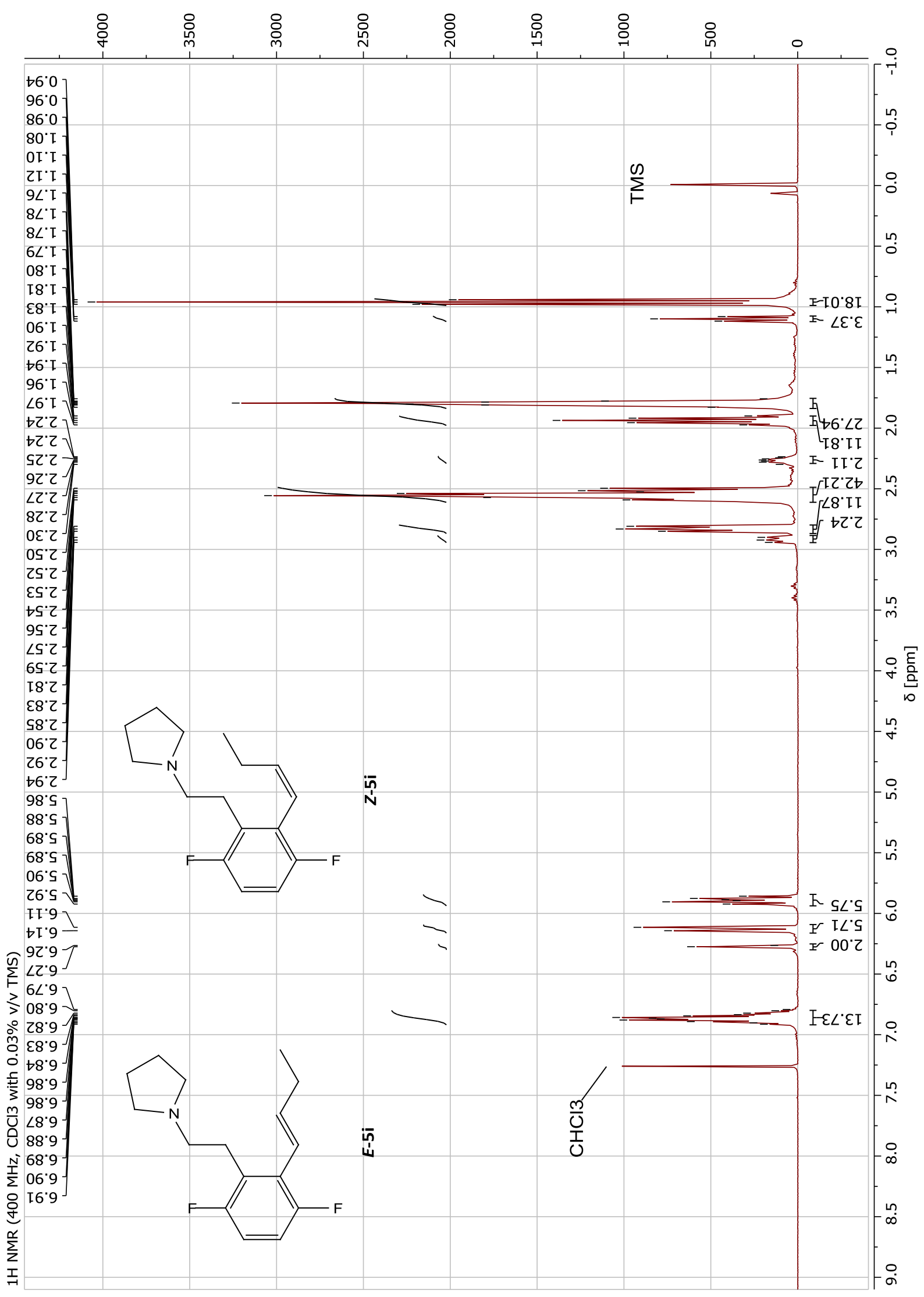


${ }^{13} \mathrm{C}\left\{{ }^{1} \mathrm{H}\right\}$ NMR of $5 i$

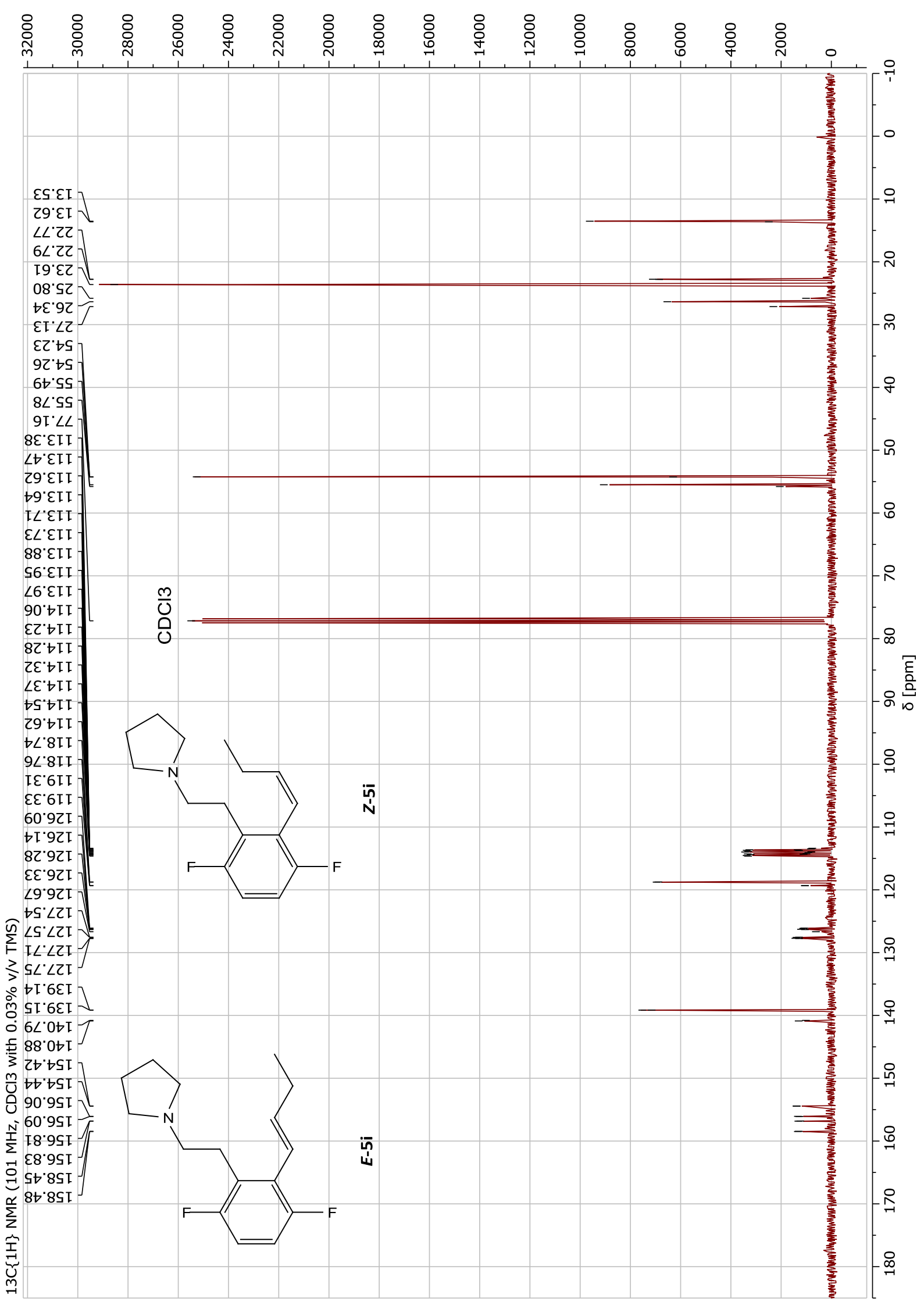


${ }^{1} \mathbf{H}$ NMR of $5 \mathbf{j}$

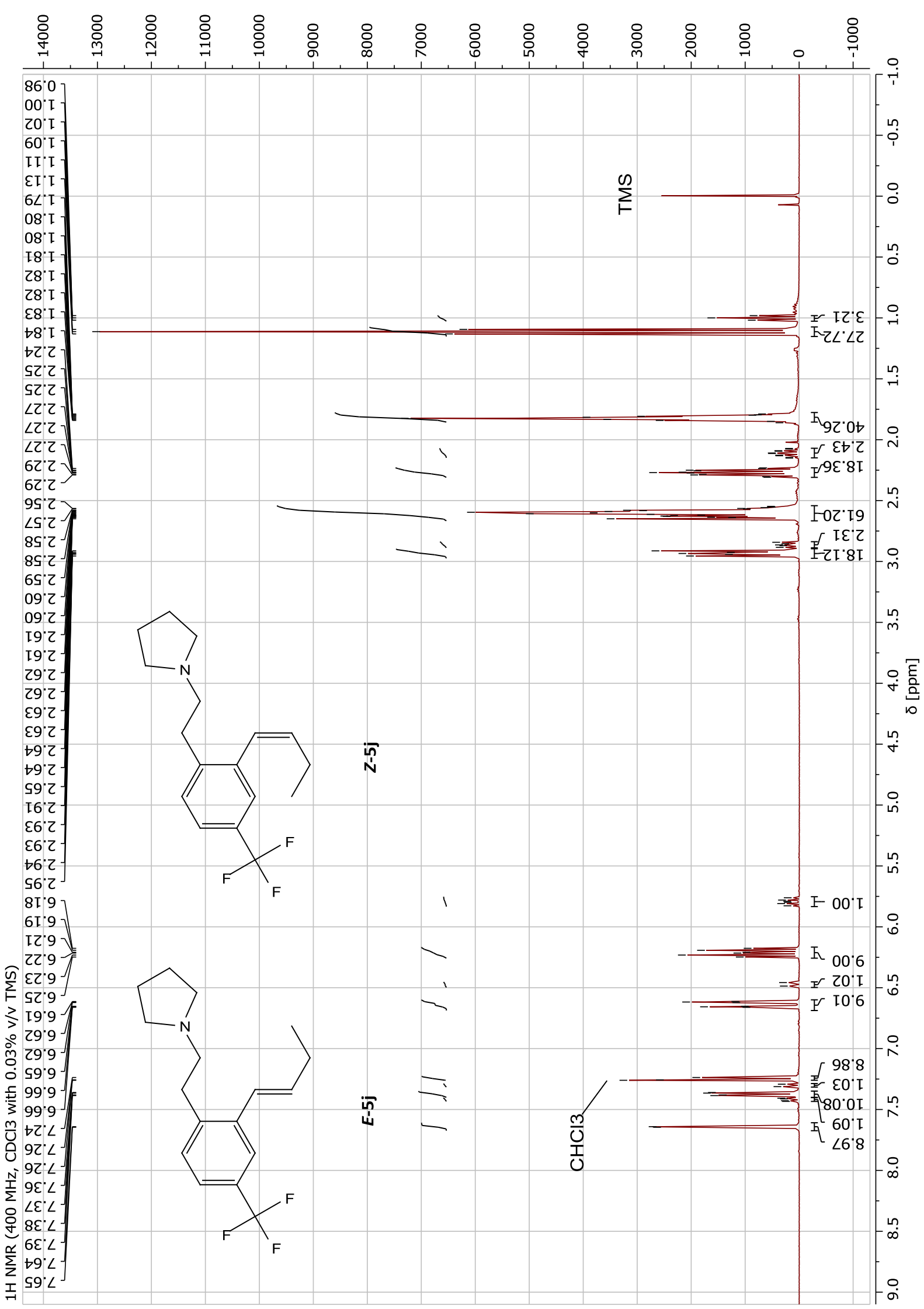


${ }^{13} \mathrm{C}\left\{{ }^{1} \mathrm{H}\right\}$ NMR of $5 \mathrm{j}$

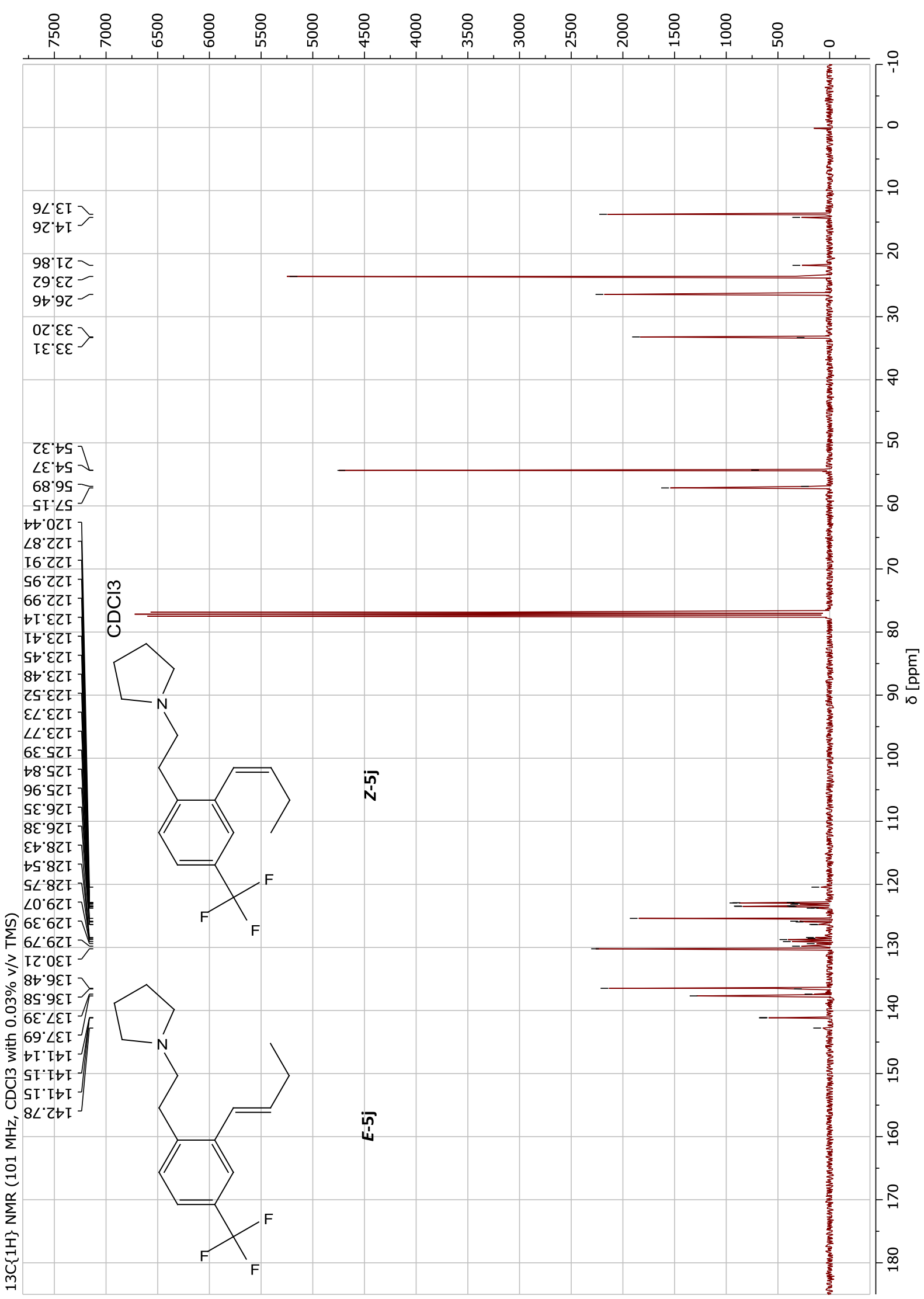


${ }^{1} \mathrm{H}$ NMR of $5 \mathrm{j}^{\prime}$

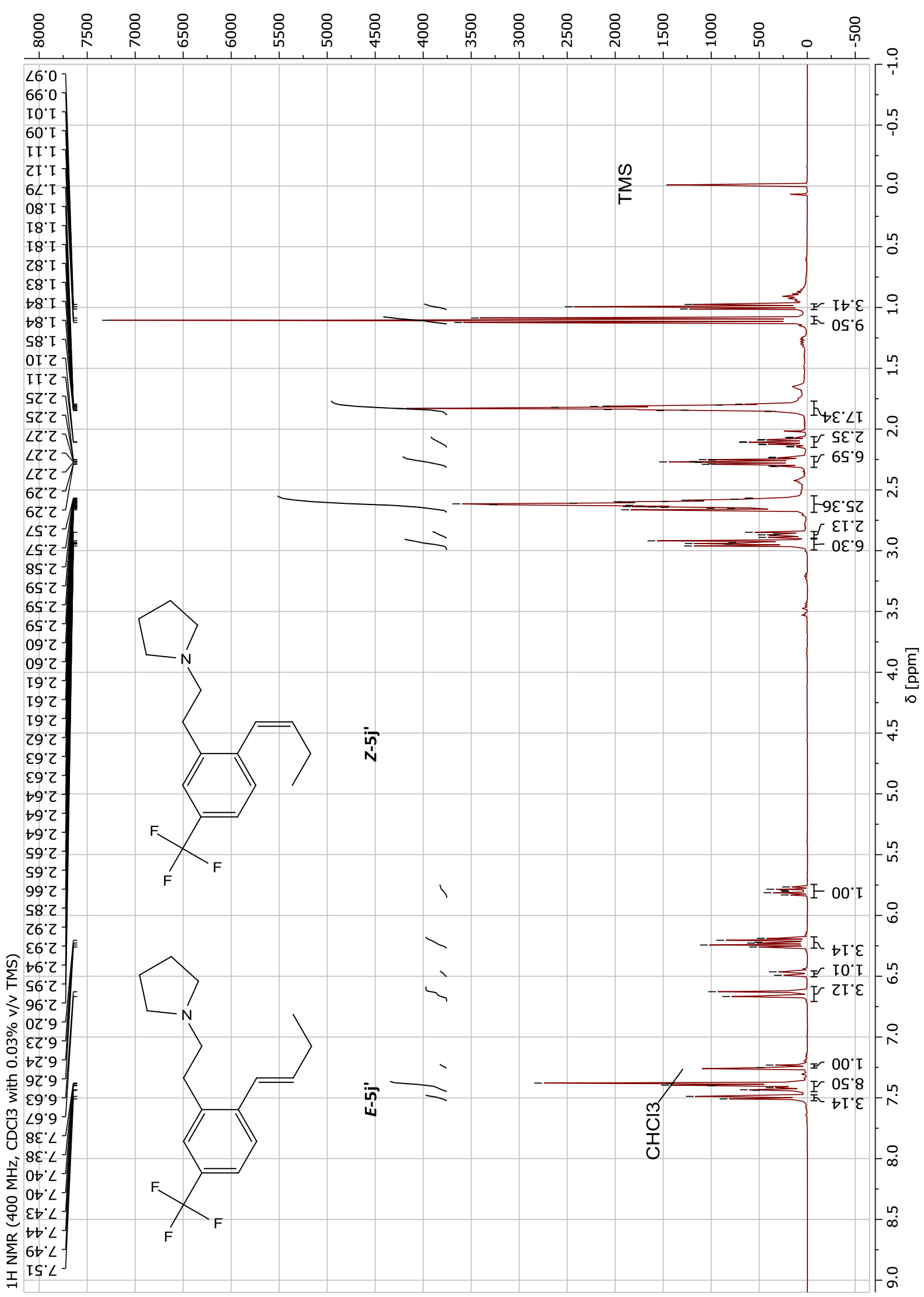


${ }^{13} \mathrm{C}\left\{{ }^{1} \mathrm{H}\right\}$ NMR of $5 j^{\prime}$

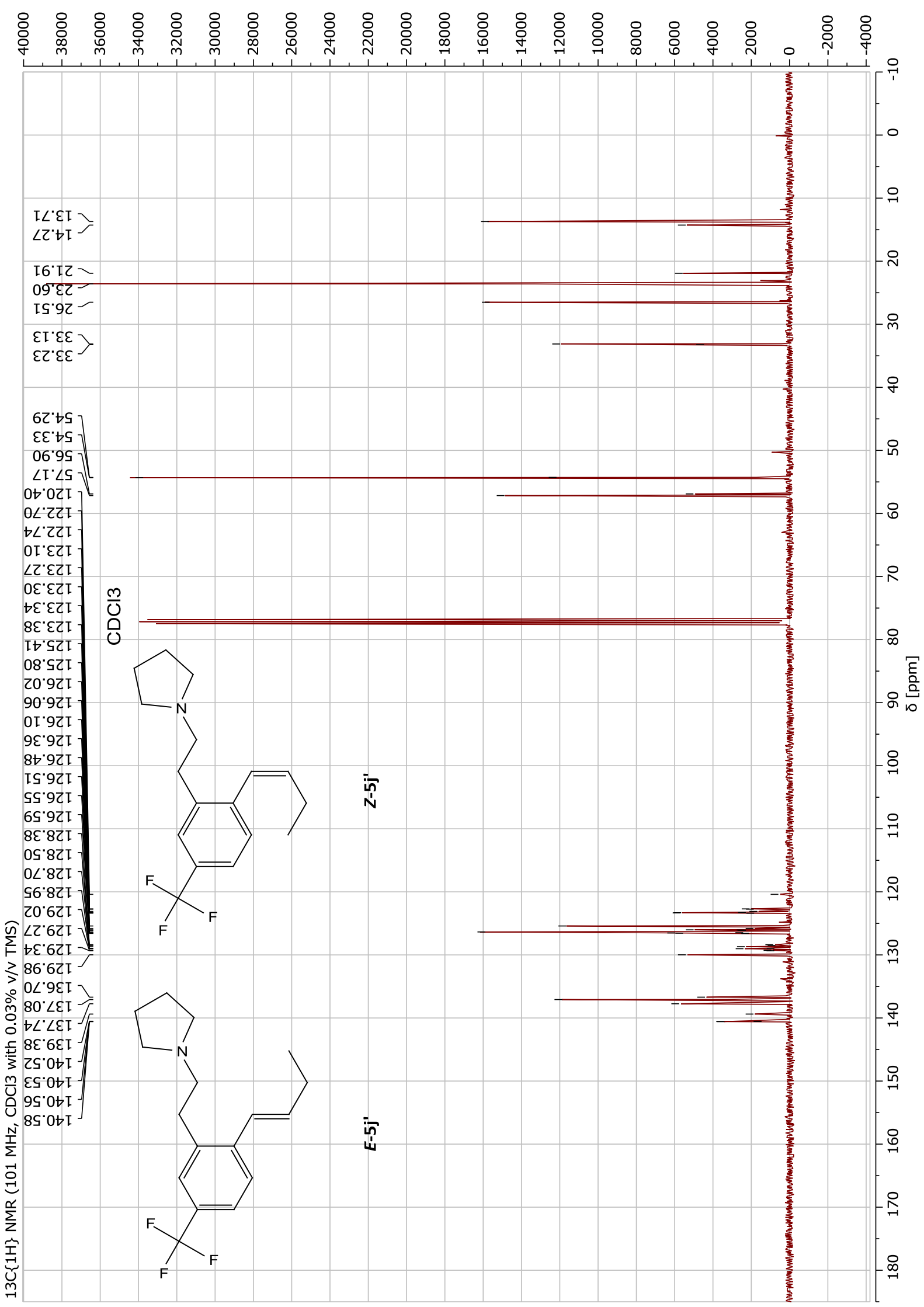


${ }^{1} \mathrm{H}$ NMR of $5 k$

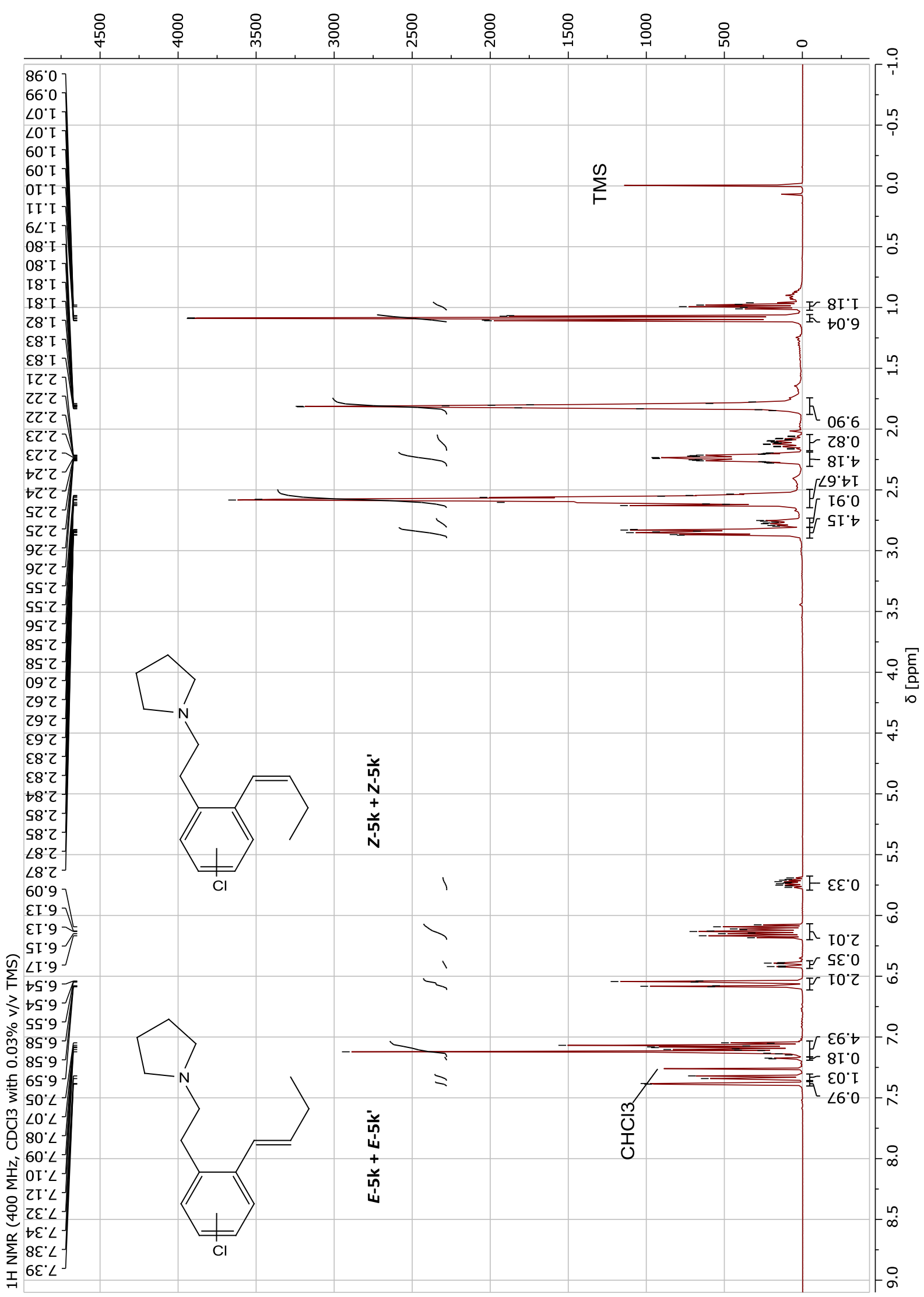


${ }^{13} \mathrm{C}\left\{{ }^{1} \mathrm{H}\right\}$ NMR $5 \mathrm{~K}$

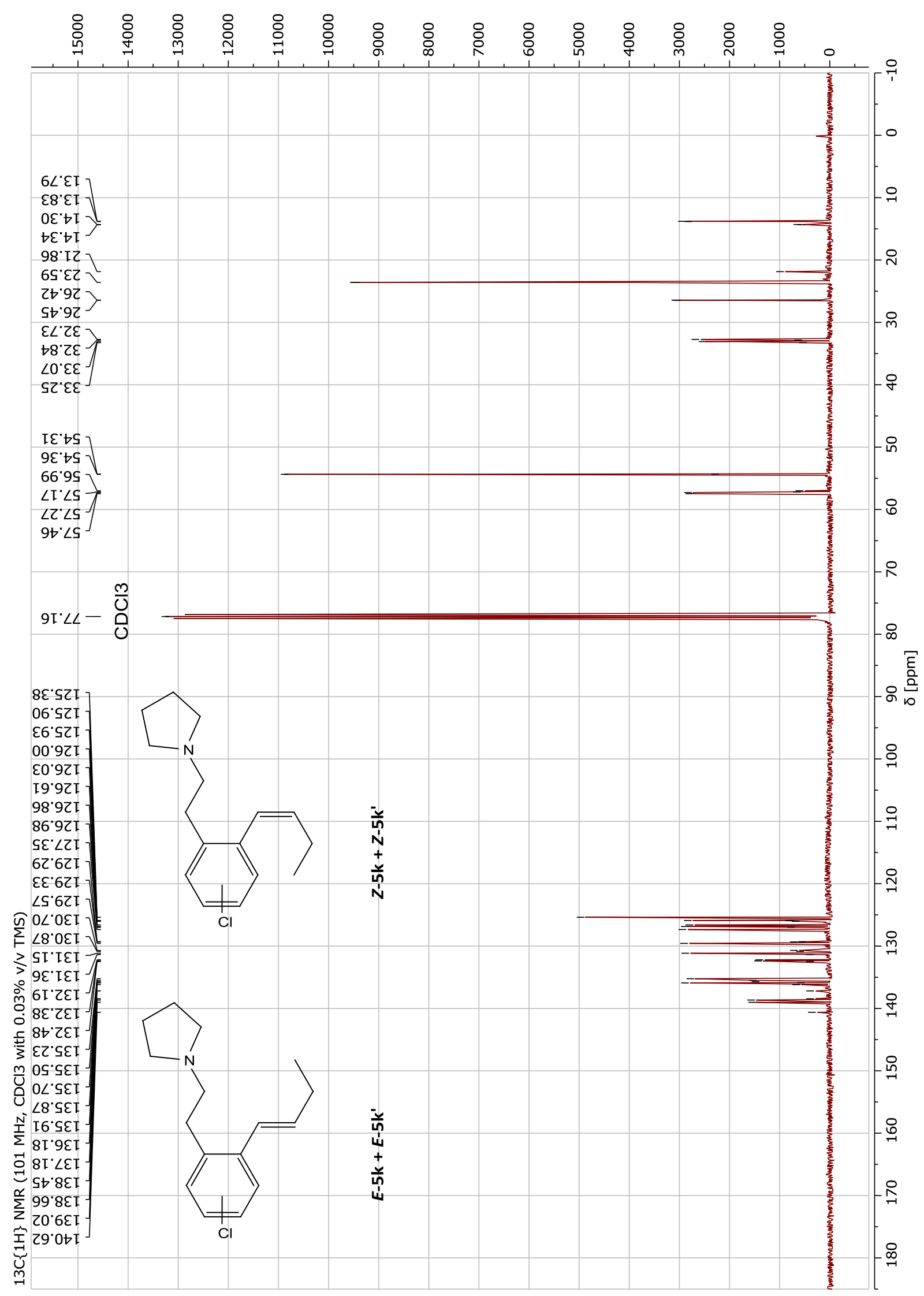


${ }^{1} \mathrm{H}$ NMR of 5 I

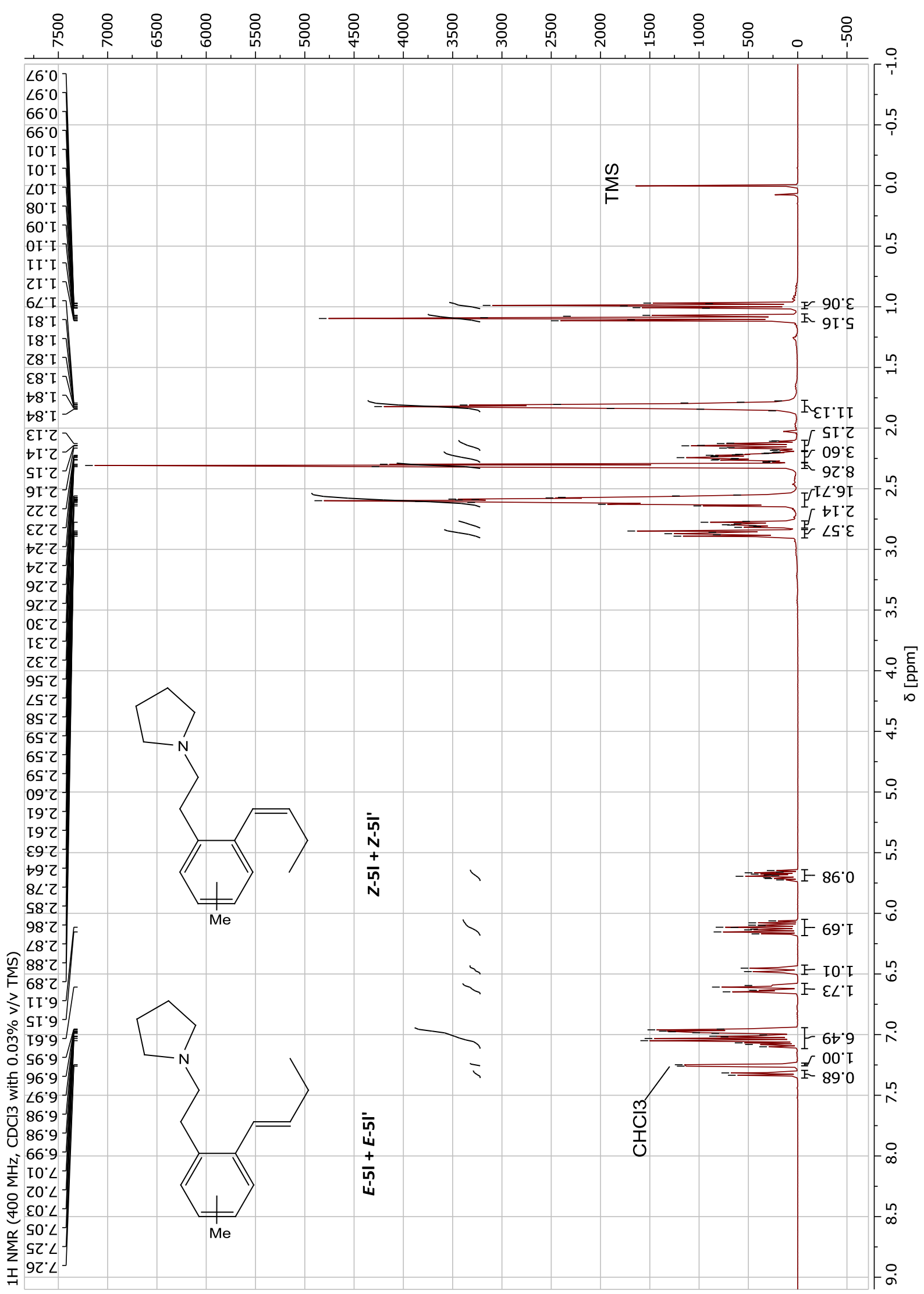


${ }^{13} \mathrm{C}\left\{{ }^{1} \mathrm{H}\right\}$ NMR of 5 I

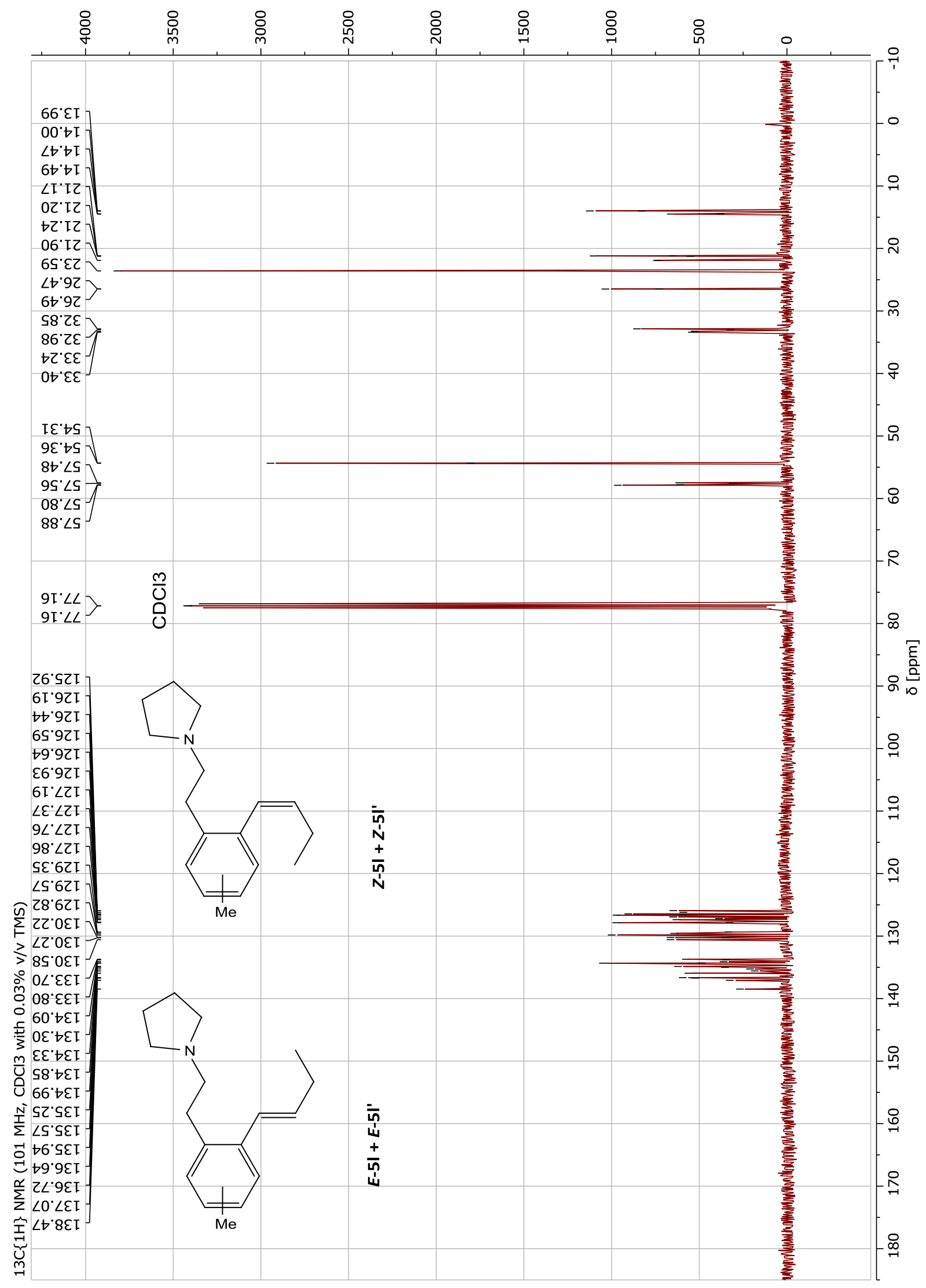


${ }^{1} \mathrm{H}$ NMR of $5 \mathrm{~m}$

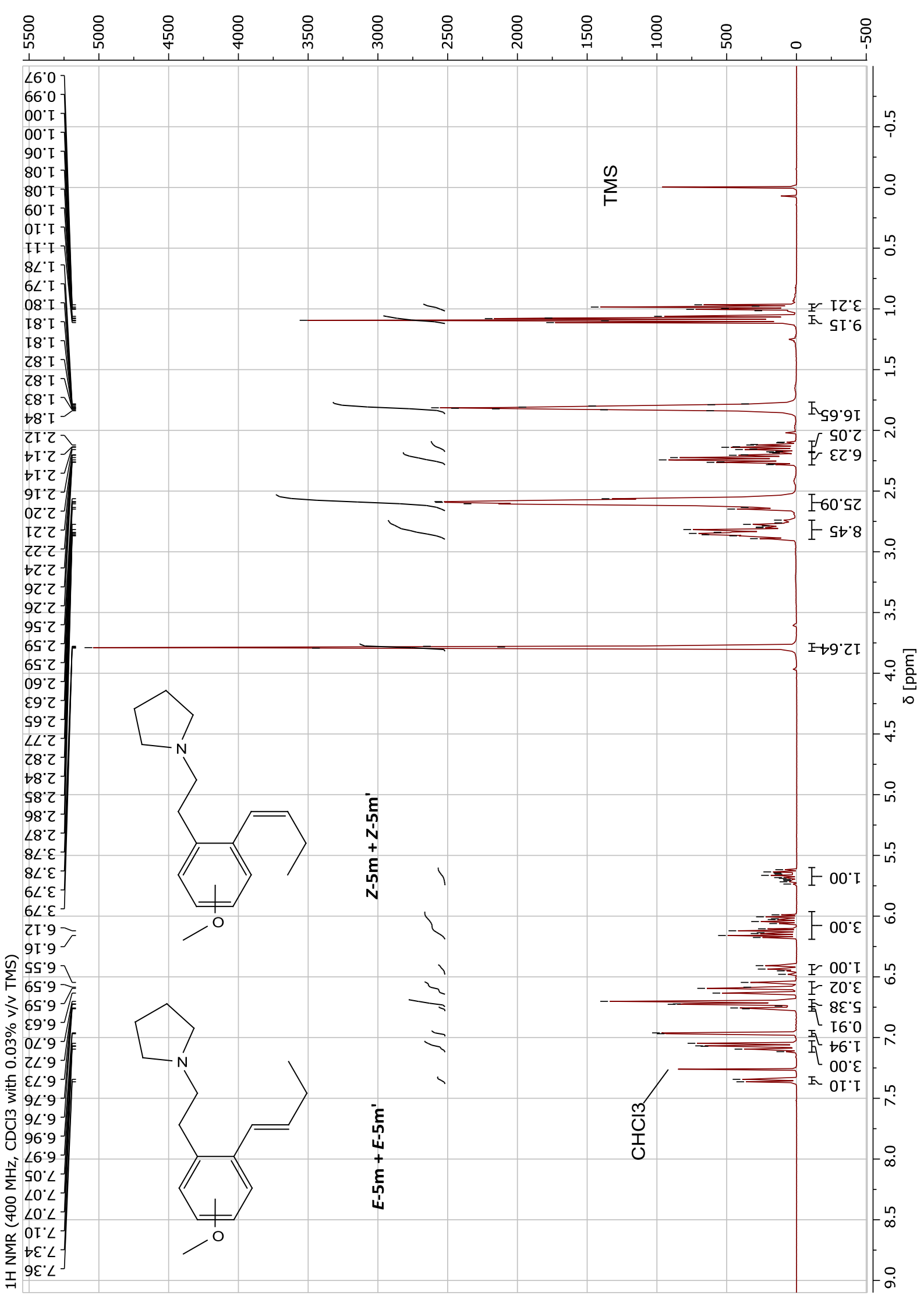


${ }^{13} \mathrm{C}\left\{{ }^{1} \mathrm{H}\right\}$ NMR of $5 \mathrm{~m}$

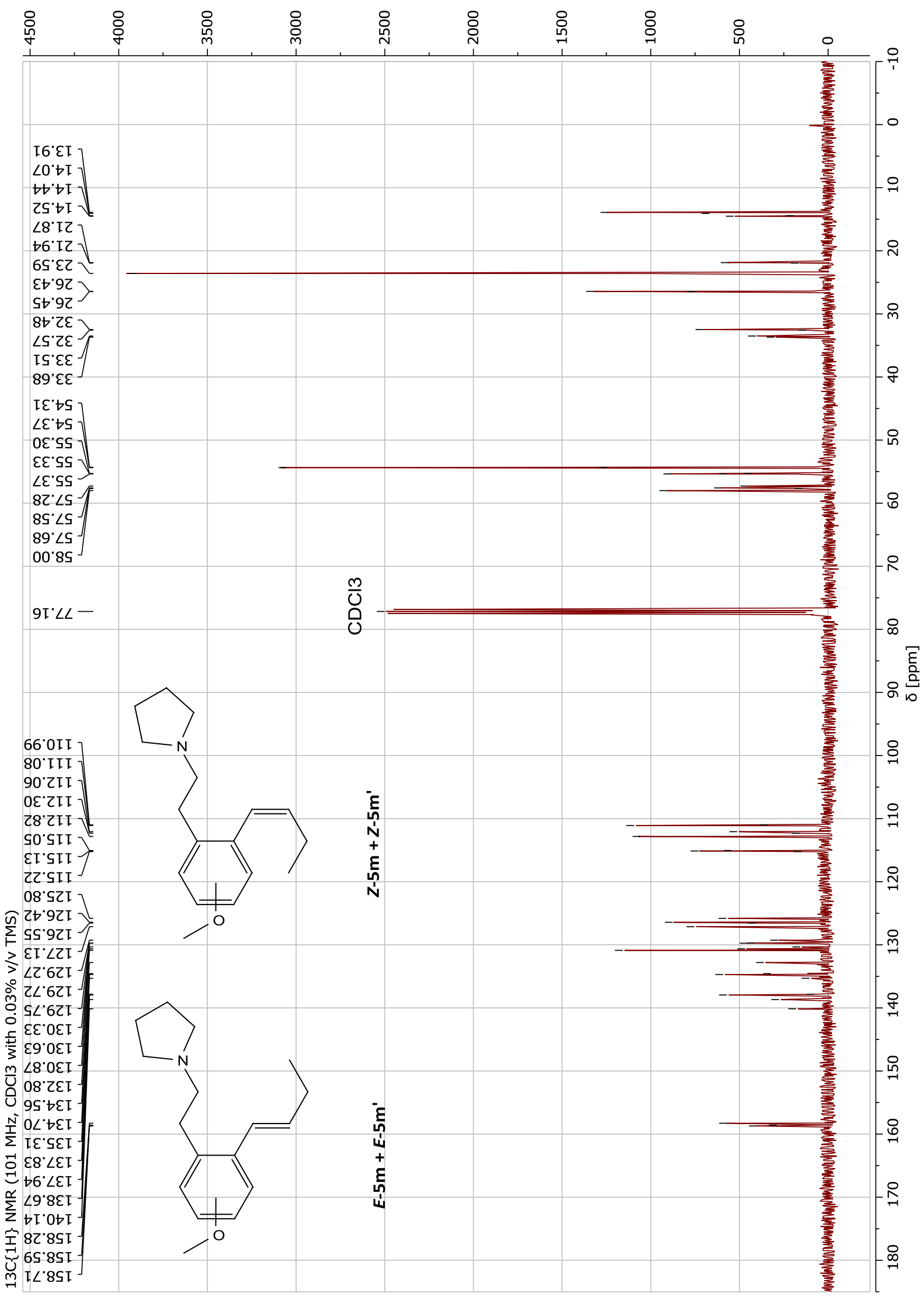


${ }^{1} \mathrm{H}$ NMR of $4 a$

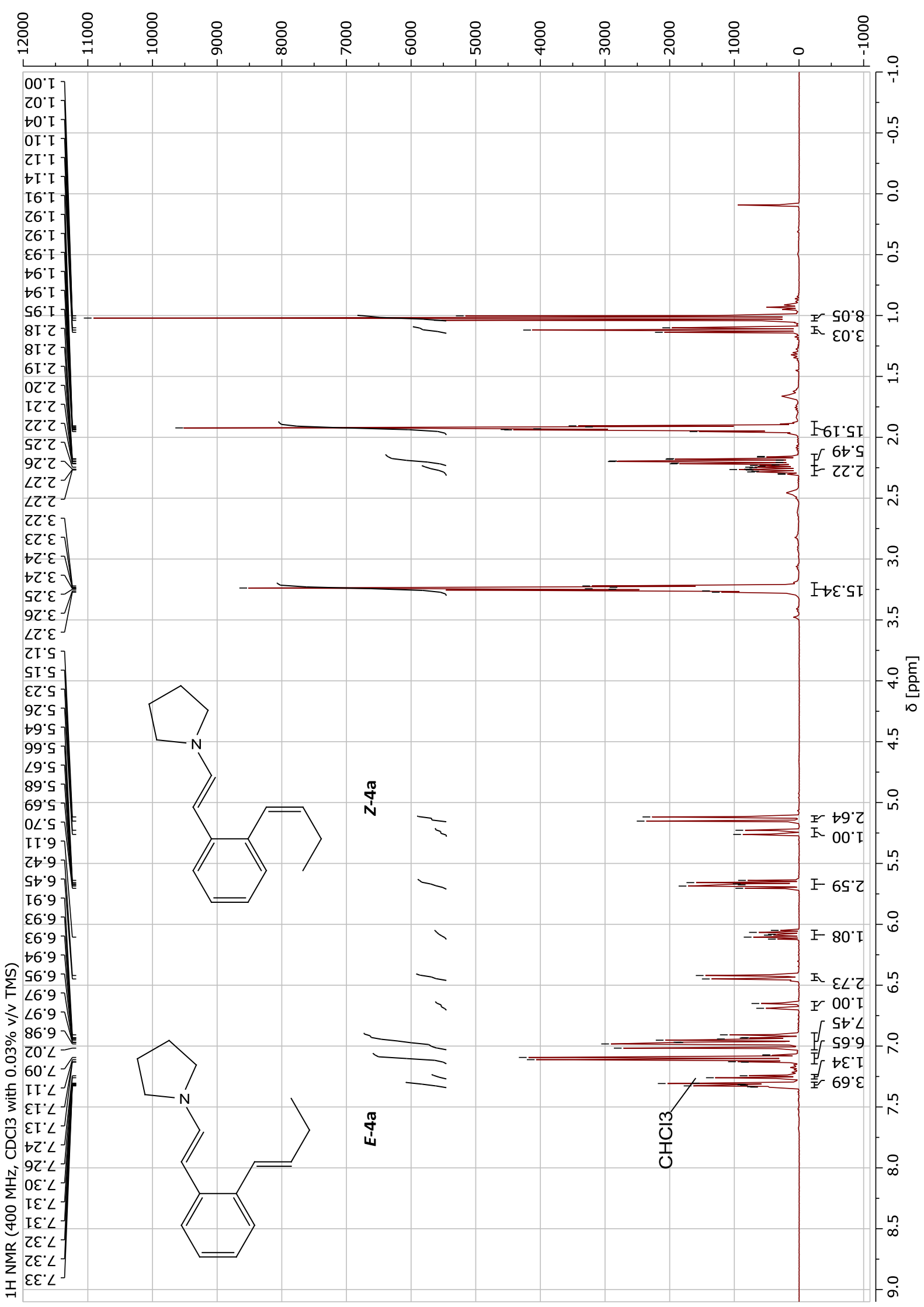


${ }^{13} \mathrm{C}\left\{{ }^{1} \mathrm{H}\right\}$ NMR of $4 a$

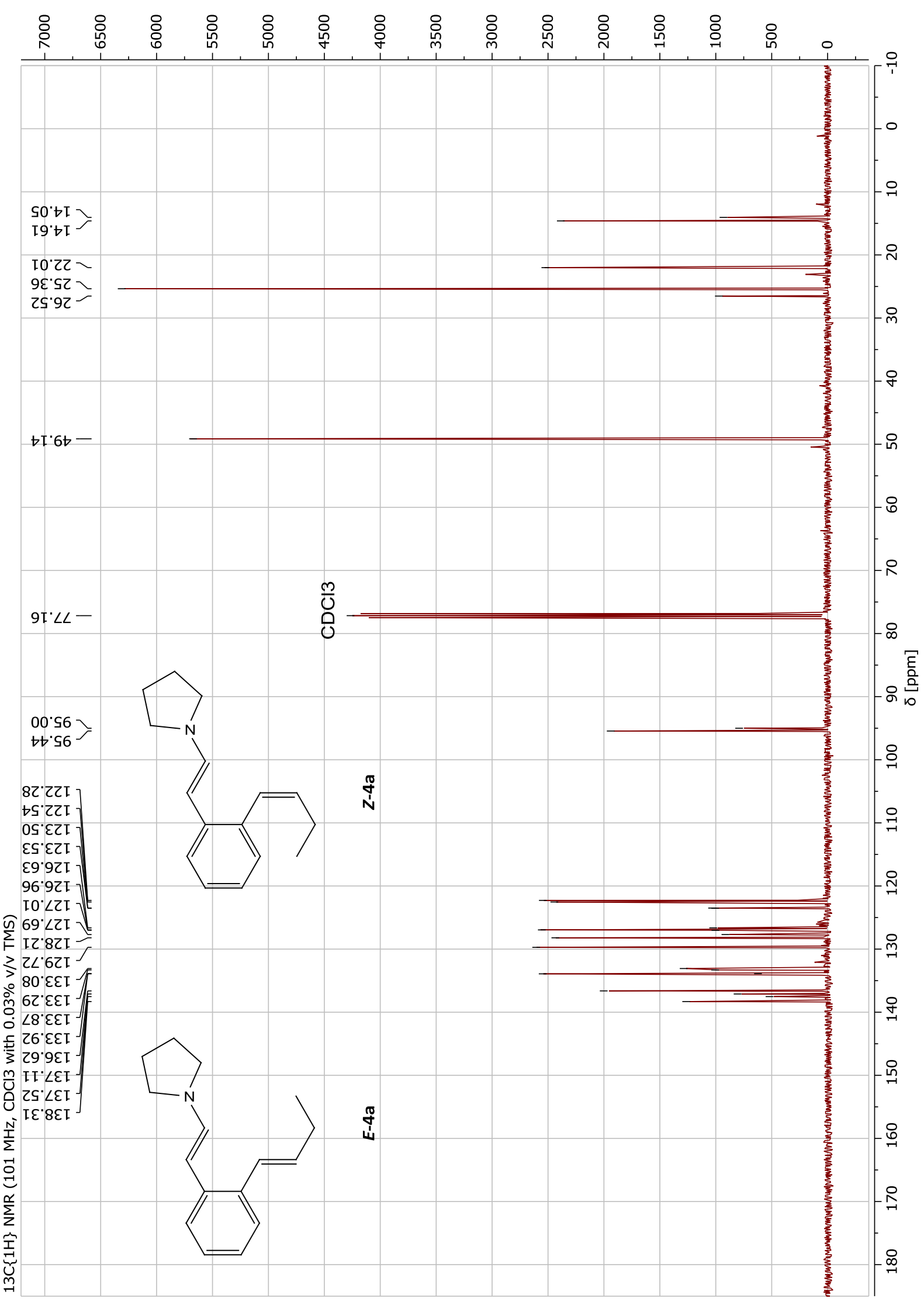




\section{Computations:}

All computations were carried out with the Gaussian 16 software package. ${ }^{7}$ Geometry optimizations were performed on a B3LYP-D3 level using the def2-TZVP basis set with the IEFPCM solvent model. ${ }^{8-11}$ Additionally, the opt=tight and int=ultrafine keywords were applied. The absence of imaginary frequencies in ground state was checked for every optimized geometry. All possible orientations of the substituents were taken into consideration. For each structure the substituent arrangement with the lowest energy was used. Molecular structures were visualized using the CYLview software. ${ }^{12}$ For the time-dependent DFT computations also the previously optimized B3LYP structures were used. ${ }^{13}$ Therefore, the Cam-B3LYP method with the cc-pVTZ basis set and the IEFPCM solvent model was applied. ${ }^{14-16}$ Using these parameters, the experimentally observed absorption for 0 -quinodimethane in acetonitrile solution by Trahanovsky et al. could be reproduced with high accuracy. ${ }^{17}$

Table S1: Computed vertical excitation energies of the low-lying excited state.

The values are given in $\mathrm{nm}$.

\begin{tabular}{|c|c|c|c|c|c|c|c|}
\hline Compound & \multicolumn{2}{|c|}{ o-quinodimethane } & 3a & 3i & 3m-c2 & 3m-c3 \\
\hline & $\begin{array}{c}\text { computed } \\
\text { (ACN) }\end{array}$ & $\begin{array}{c}\text { experimental } \\
\text { (ACN) }\end{array}$ & \multicolumn{4}{|c|}{ computed (THF) } \\
\hline \hline $\begin{array}{c}\text { HOMO-LUMO } \\
\text { transition }\end{array}$ & 378 & 367 & 417 & 419 & 411 & 412 \\
\hline
\end{tabular}


Table S2: Coordinates and energy parameters of $o$-quinodimethane (B3LYP-D3/def2-TZVP, IEFPCM=ACN).

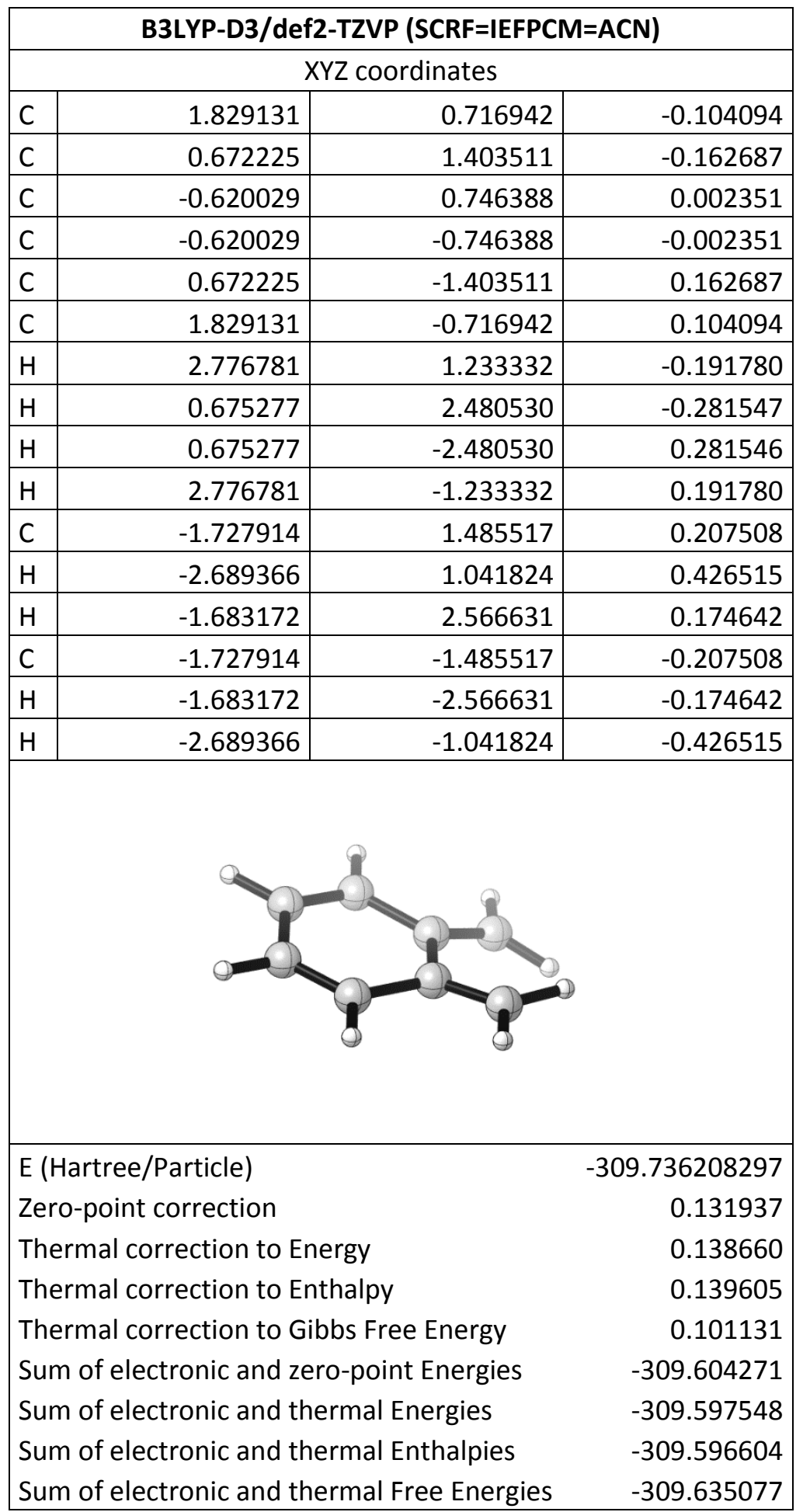


Table S3: Coordinates and energy parameters of 3a (B3LYP-D3/def2-TZVP, IEFPCM=THF).

\begin{tabular}{|c|c|c|c|}
\hline \multicolumn{4}{|c|}{ B3LYP-D3/def2-TZVP (SCRF=IEFPCM=THF) } \\
\hline \multicolumn{4}{|c|}{$\mathrm{XYZ}$ coordinates } \\
\hline C & 1.658649 & -0.931785 & 0.039550 \\
\hline $\mathrm{C}$ & 2.191582 & 0.443343 & -0.098287 \\
\hline $\mathrm{C}$ & 0.333633 & -1.141883 & -0.105875 \\
\hline C & 1.337362 & 1.489717 & -0.094451 \\
\hline $\mathrm{H}$ & -0.060693 & -2.149048 & -0.041269 \\
\hline $\mathrm{H}$ & 1.735275 & 2.497105 & -0.137053 \\
\hline $\mathrm{C}$ & 4.461284 & -0.443832 & 0.013994 \\
\hline $\mathrm{H}$ & 5.534628 & -0.302735 & -0.011243 \\
\hline $\mathrm{C}$ & 3.937926 & -1.775127 & 0.249375 \\
\hline $\mathrm{H}$ & 4.635200 & -2.589827 & 0.400380 \\
\hline $\mathrm{C}$ & 3.632157 & 0.606719 & -0.155457 \\
\hline C & 2.608734 & -2.004722 & 0.267278 \\
\hline $\mathrm{H}$ & 2.220088 & -3.004781 & 0.421561 \\
\hline $\mathrm{H}$ & 4.025835 & 1.605002 & -0.308627 \\
\hline$C$ & -0.963197 & 2.500154 & -0.437346 \\
\hline $\mathrm{H}$ & -0.710867 & 2.653317 & -1.491950 \\
\hline $\mathrm{H}$ & -2.016935 & 2.219762 & -0.407310 \\
\hline$C$ & -0.600859 & -0.023099 & -0.512064 \\
\hline C & -0.145342 & 1.315469 & 0.096906 \\
\hline $\mathrm{H}$ & -0.312923 & 1.262361 & 1.186887 \\
\hline $\mathrm{N}$ & -2.015064 & -0.283696 & -0.264142 \\
\hline $\mathrm{C}$ & -2.628811 & -1.328465 & -1.076884 \\
\hline $\mathrm{H}$ & -2.114321 & -2.299445 & -0.975826 \\
\hline $\mathrm{H}$ & -2.605332 & -1.052929 & -2.133106 \\
\hline$C$ & -4.045295 & -1.426878 & -0.503106 \\
\hline $\mathrm{H}$ & -4.465018 & -2.425635 & -0.622953 \\
\hline $\mathrm{H}$ & -4.704533 & -0.727048 & -1.017447 \\
\hline $\mathrm{C}$ & -3.881808 & -1.020258 & 0.984962 \\
\hline $\mathrm{H}$ & -4.095033 & -1.846807 & 1.662829 \\
\hline $\mathrm{H}$ & -4.562199 & -0.207927 & 1.240014 \\
\hline $\mathrm{C}$ & -2.415203 & -0.568449 & 1.112564 \\
\hline $\mathrm{H}$ & -2.298835 & 0.314026 & 1.742910 \\
\hline $\mathrm{H}$ & -1.803853 & -1.370356 & 1.558308 \\
\hline$C$ & -0.757468 & 3.796776 & 0.343394 \\
\hline $\mathrm{H}$ & -1.028883 & 3.664370 & 1.394005 \\
\hline $\mathrm{H}$ & 0.278389 & 4.140899 & 0.311241 \\
\hline $\mathrm{H}$ & -1.379155 & 4.597112 & -0.062767 \\
\hline $\mathrm{H}$ & -0.505453 & 0.082374 & -1.606900 \\
\hline
\end{tabular}




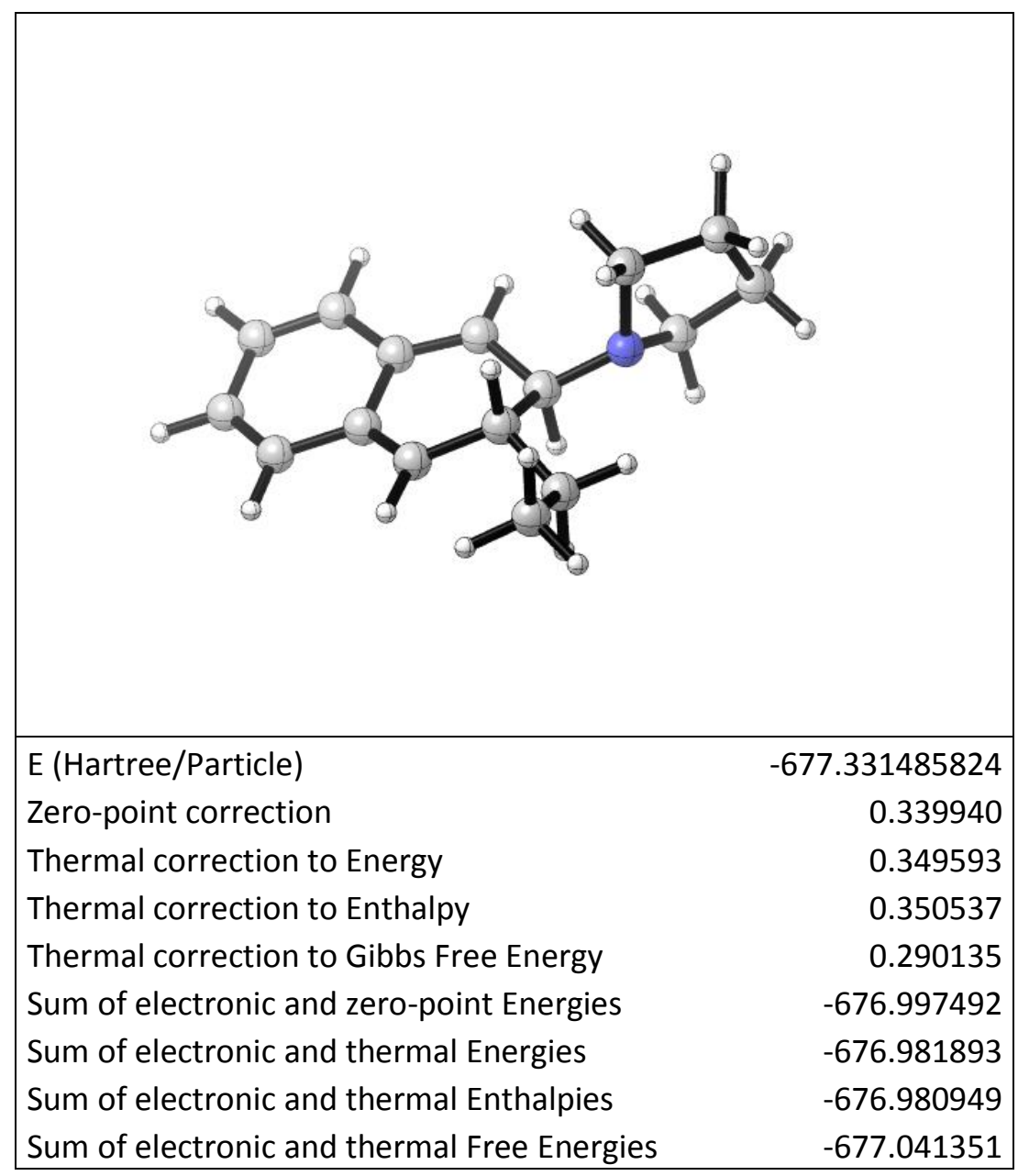


Table S4: Coordinates and energy parameters of 3i (B3LYP-D3/def2-TZVP, IEFPCM=THF).

\begin{tabular}{|c|c|c|c|}
\hline \multicolumn{4}{|c|}{ B3LYP-D3/def2-TZVP (SCRF=IEFPCM=THF) } \\
\hline \multicolumn{4}{|c|}{ XYZ coordinates } \\
\hline C & 1.278764 & -0.873349 & -0.006287 \\
\hline C & 1.836254 & 0.496499 & -0.072046 \\
\hline C & -0.045154 & -1.060521 & -0.166201 \\
\hline C & 1.008478 & 1.559522 & -0.025392 \\
\hline $\mathrm{H}$ & -0.451345 & -2.062411 & -0.149242 \\
\hline $\mathrm{H}$ & 1.429004 & 2.555902 & -0.014181 \\
\hline C & 4.105600 & -0.441639 & 0.023227 \\
\hline $\mathrm{H}$ & 5.176077 & -0.292784 & 0.021649 \\
\hline C & 3.557831 & -1.773684 & 0.175306 \\
\hline $\mathrm{H}$ & 4.218657 & -2.622306 & 0.281927 \\
\hline C & 3.278065 & 0.607140 & -0.099427 \\
\hline C & 2.228073 & -1.950463 & 0.167866 \\
\hline C & -1.266470 & 2.620021 & -0.364858 \\
\hline $\mathrm{H}$ & -0.999823 & 2.802163 & -1.411086 \\
\hline $\mathrm{H}$ & -2.324841 & 2.357406 & -0.354140 \\
\hline C & -0.956926 & 0.094253 & -0.521071 \\
\hline C & -0.478113 & 1.401785 & 0.138630 \\
\hline $\mathrm{H}$ & -0.656403 & 1.317450 & 1.224384 \\
\hline$N$ & -2.372933 & -0.150243 & -0.279722 \\
\hline C & -3.010320 & -1.145337 & -1.136354 \\
\hline $\mathrm{H}$ & -2.515947 & -2.130204 & -1.080486 \\
\hline $\mathrm{H}$ & -2.984512 & -0.822966 & -2.179082 \\
\hline $\mathrm{C}$ & -4.425706 & -1.238577 & -0.561079 \\
\hline $\mathrm{H}$ & -4.872842 & -2.217380 & -0.733714 \\
\hline $\mathrm{H}$ & -5.067118 & -0.493231 & -1.032186 \\
\hline C & -4.244853 & -0.919507 & 0.945862 \\
\hline $\mathrm{H}$ & -4.456240 & -1.783453 & 1.575808 \\
\hline $\mathrm{H}$ & -4.917725 & -0.120110 & 1.255441 \\
\hline C & -2.773227 & -0.486470 & 1.086124 \\
\hline $\mathrm{H}$ & -2.646928 & 0.369023 & 1.750835 \\
\hline $\mathrm{H}$ & -2.168255 & -1.311075 & 1.497383 \\
\hline C & -1.044973 & 3.885580 & 0.460767 \\
\hline $\mathrm{H}$ & -1.329557 & 3.723770 & 1.503630 \\
\hline $\mathrm{H}$ & -0.003206 & 4.212625 & 0.449430 \\
\hline $\mathrm{H}$ & -1.648398 & 4.709271 & 0.074724 \\
\hline $\mathrm{H}$ & -0.858825 & 0.237434 & -1.610816 \\
\hline $\mathrm{F}$ & 1.707165 & -3.195324 & 0.287110 \\
\hline $\mathrm{F}$ & 3.782259 & 1.858775 & -0.218513 \\
\hline
\end{tabular}




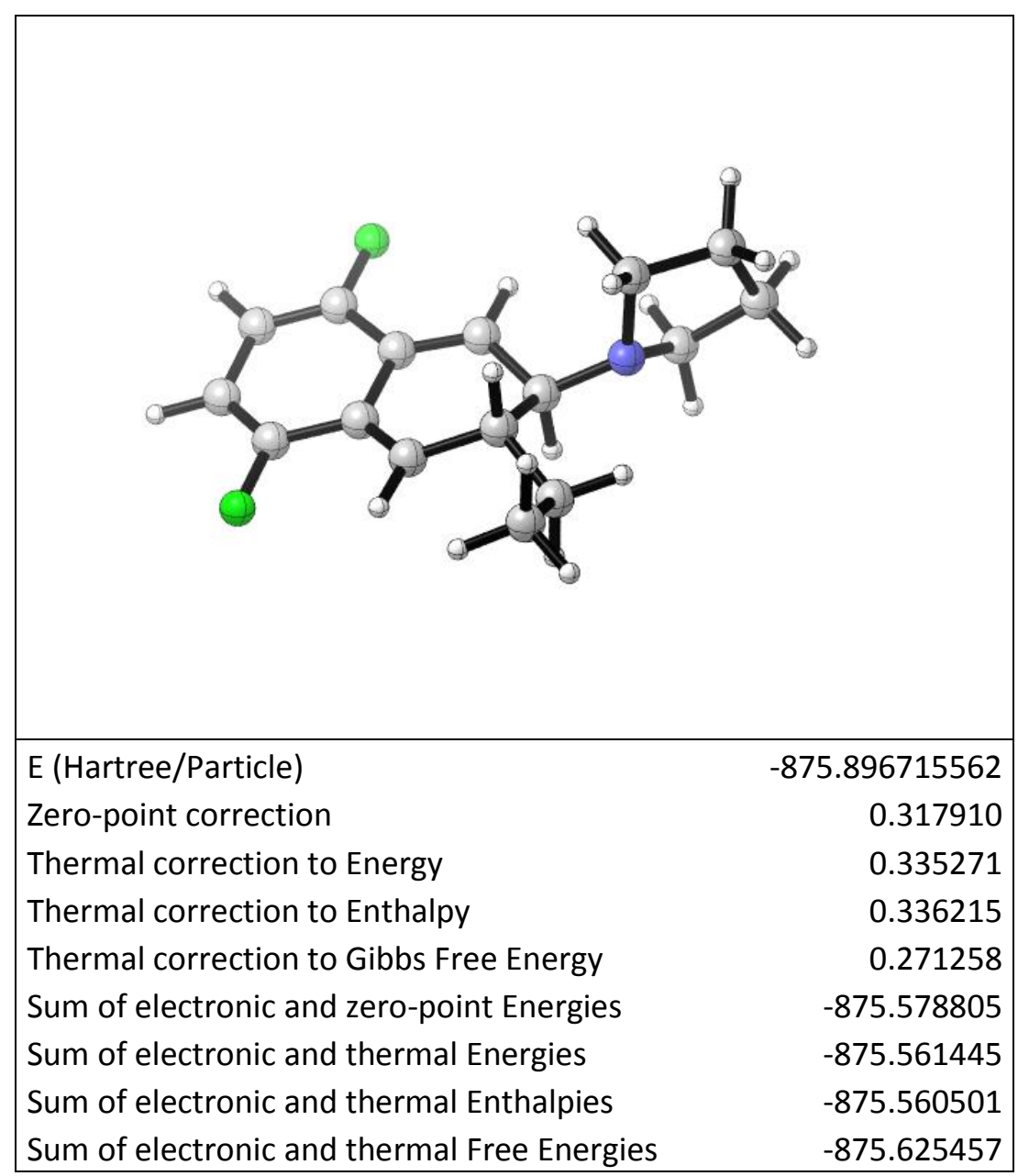


Table S5: Coordinates and energy parameters of 3m-c2 (B3LYP-D3/def2-TZVP, IEFPCM=THF).

\begin{tabular}{|c|c|c|c|}
\hline \multicolumn{4}{|c|}{ B3LYP-D3/def2-TZVP (SCRF=IEFPCM=THF) } \\
\hline \multicolumn{4}{|c|}{ XYZ coordinates } \\
\hline C & 1.229209 & -0.164485 & -0.091448 \\
\hline $\mathrm{C}$ & 1.306504 & 1.313241 & -0.151207 \\
\hline C & 0.023958 & -0.757254 & -0.225467 \\
\hline C & 0.177873 & 2.048870 & -0.071141 \\
\hline $\mathrm{H}$ & -0.044589 & -1.838556 & -0.225591 \\
\hline $\mathrm{H}$ & 0.252500 & 3.130286 & -0.061275 \\
\hline C & 3.738711 & 1.172720 & -0.124615 \\
\hline $\mathrm{H}$ & 4.724833 & 1.617838 & -0.152346 \\
\hline C & 3.658881 & -0.268984 & 0.036892 \\
\hline C & 2.623277 & 1.917533 & -0.218478 \\
\hline $\mathrm{C}$ & 2.466012 & -0.908867 & 0.059087 \\
\hline $\mathrm{H}$ & 2.399510 & -1.983526 & 0.151318 \\
\hline $\mathrm{H}$ & 2.688551 & 2.994719 & -0.316959 \\
\hline$C$ & -2.327396 & 2.331427 & -0.330567 \\
\hline $\mathrm{H}$ & -2.163452 & 2.602420 & -1.378901 \\
\hline $\mathrm{H}$ & -3.246448 & 1.744590 & -0.300732 \\
\hline$C$ & -1.223965 & 0.040076 & -0.540017 \\
\hline C & -1.176015 & 1.425466 & 0.129654 \\
\hline $\mathrm{H}$ & -1.287358 & 1.275263 & 1.217496 \\
\hline $\mathrm{N}$ & -2.479156 & -0.653084 & -0.259331 \\
\hline $\mathrm{C}$ & -2.787892 & -1.793306 & -1.115445 \\
\hline $\mathrm{H}$ & -1.998263 & -2.563927 & -1.093554 \\
\hline $\mathrm{H}$ & -2.903515 & -1.471393 & -2.152347 \\
\hline C & -4.076198 & -2.349938 & -0.502100 \\
\hline $\mathrm{H}$ & -4.177063 & -3.422299 & -0.669573 \\
\hline $\mathrm{H}$ & -4.942804 & -1.862402 & -0.949653 \\
\hline C & -3.968758 & -1.985844 & 1.002062 \\
\hline $\mathrm{H}$ & -3.889693 & -2.870043 & 1.634703 \\
\hline $\mathrm{H}$ & -4.848490 & -1.431284 & 1.328453 \\
\hline C & -2.701593 & -1.117395 & 1.108599 \\
\hline $\mathrm{H}$ & -2.823516 & -0.275813 & 1.791625 \\
\hline $\mathrm{H}$ & -1.854621 & -1.717782 & 1.479129 \\
\hline C & -2.500463 & 3.592246 & 0.514283 \\
\hline $\mathrm{H}$ & -2.688417 & 3.335836 & 1.560182 \\
\hline $\mathrm{H}$ & -1.618406 & 4.235441 & 0.485890 \\
\hline $\mathrm{H}$ & -3.346382 & 4.184338 & 0.159455 \\
\hline $\mathrm{H}$ & -1.225368 & 0.218945 & -1.629166 \\
\hline 0 & 4.881715 & -0.853371 & 0.144606 \\
\hline C & 4.936242 & -2.266476 & 0.308091 \\
\hline $\mathrm{H}$ & 4.494892 & -2.779440 & -0.551059 \\
\hline $\mathrm{H}$ & 5.990338 & -2.521707 & 0.382692 \\
\hline $\mathrm{H}$ & 4.417818 & -2.577128 & 1.219572 \\
\hline
\end{tabular}




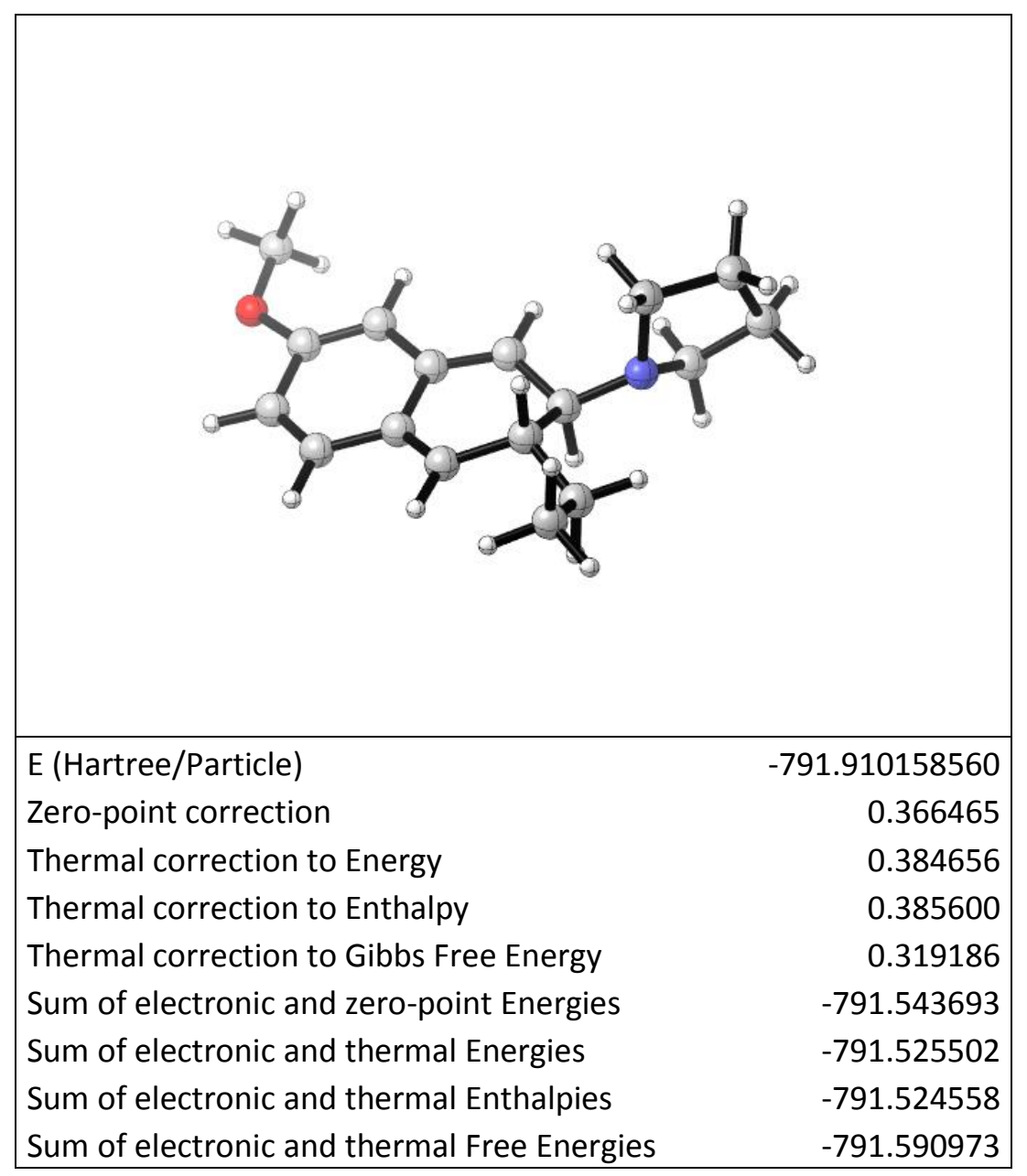


Table S6: Coordinates and energy parameters of 3m-c3 (B3LYP-D3/def2-TZVP, IEFPCM=THF).

\begin{tabular}{|c|c|c|c|}
\hline \multicolumn{4}{|c|}{ B3LYP-D3/def2-TZVP (SCRF=IEFPCM=THF) } \\
\hline \multicolumn{4}{|c|}{ XYZ coordinates } \\
\hline C & 0.914047 & -0.991869 & 0.059828 \\
\hline C & 1.498044 & 0.363823 & -0.057713 \\
\hline C & -0.413695 & -1.161457 & -0.102755 \\
\hline C & 0.673176 & 1.433941 & -0.055031 \\
\hline $\mathrm{H}$ & -0.837849 & -2.157127 & -0.049252 \\
\hline $\mathrm{H}$ & 1.099982 & 2.429963 & -0.079446 \\
\hline C & 3.728904 & -0.606056 & 0.058682 \\
\hline C & 3.154728 & -1.922120 & 0.280142 \\
\hline $\mathrm{H}$ & 3.840031 & -2.747028 & 0.426152 \\
\hline C & 2.943605 & 0.483981 & -0.107333 \\
\hline C & 1.822026 & -2.100244 & 0.286264 \\
\hline $\mathrm{H}$ & 1.399835 & -3.087818 & 0.430195 \\
\hline $\mathrm{H}$ & 3.363237 & 1.470412 & -0.243884 \\
\hline C & -1.589473 & 2.517064 & -0.426372 \\
\hline $\mathrm{H}$ & -1.316344 & 2.663655 & -1.476842 \\
\hline $\mathrm{H}$ & -2.652437 & 2.271966 & -0.411954 \\
\hline C & -1.310758 & -0.012617 & -0.505748 \\
\hline C & -0.818371 & 1.305711 & 0.117424 \\
\hline $\mathrm{H}$ & -1.010232 & 1.254457 & 1.203261 \\
\hline $\mathrm{N}$ & -2.734294 & -0.235170 & -0.271290 \\
\hline C & -3.371662 & -1.250709 & -1.102617 \\
\hline $\mathrm{H}$ & -2.886010 & -2.237531 & -1.011216 \\
\hline $\mathrm{H}$ & -3.332183 & -0.961901 & -2.154818 \\
\hline C & -4.794883 & -1.316336 & -0.540704 \\
\hline $\mathrm{H}$ & -5.241375 & -2.301415 & -0.676134 \\
\hline $\mathrm{H}$ & -5.430310 & -0.592095 & -1.051201 \\
\hline C & -4.631742 & -0.932677 & 0.953517 \\
\hline $\mathrm{H}$ & -4.876181 & -1.760117 & 1.619684 \\
\hline $\mathrm{H}$ & -5.288902 & -0.102681 & 1.212646 \\
\hline C & -3.153170 & -0.527651 & 1.098272 \\
\hline $\mathrm{H}$ & -3.014418 & 0.341348 & 1.742437 \\
\hline $\mathrm{H}$ & -2.570304 & -1.354669 & 1.536388 \\
\hline C & -1.353569 & 3.805691 & 0.359221 \\
\hline $\mathrm{H}$ & -1.641248 & 3.679868 & 1.406375 \\
\hline $\mathrm{H}$ & -0.307276 & 4.117517 & 0.339906 \\
\hline $\mathrm{H}$ & -1.945674 & 4.625606 & -0.052312 \\
\hline $\mathrm{H}$ & -1.202341 & 0.098543 & -1.598622 \\
\hline 0 & 5.088748 & -0.620232 & 0.055381 \\
\hline C & 5.769926 & 0.612009 & -0.154315 \\
\hline $\mathrm{H}$ & 6.831446 & 0.378756 & -0.129440 \\
\hline $\mathrm{H}$ & 5.511597 & 1.043858 & -1.125360 \\
\hline $\mathrm{H}$ & 5.534233 & 1.331571 & 0.634855 \\
\hline
\end{tabular}




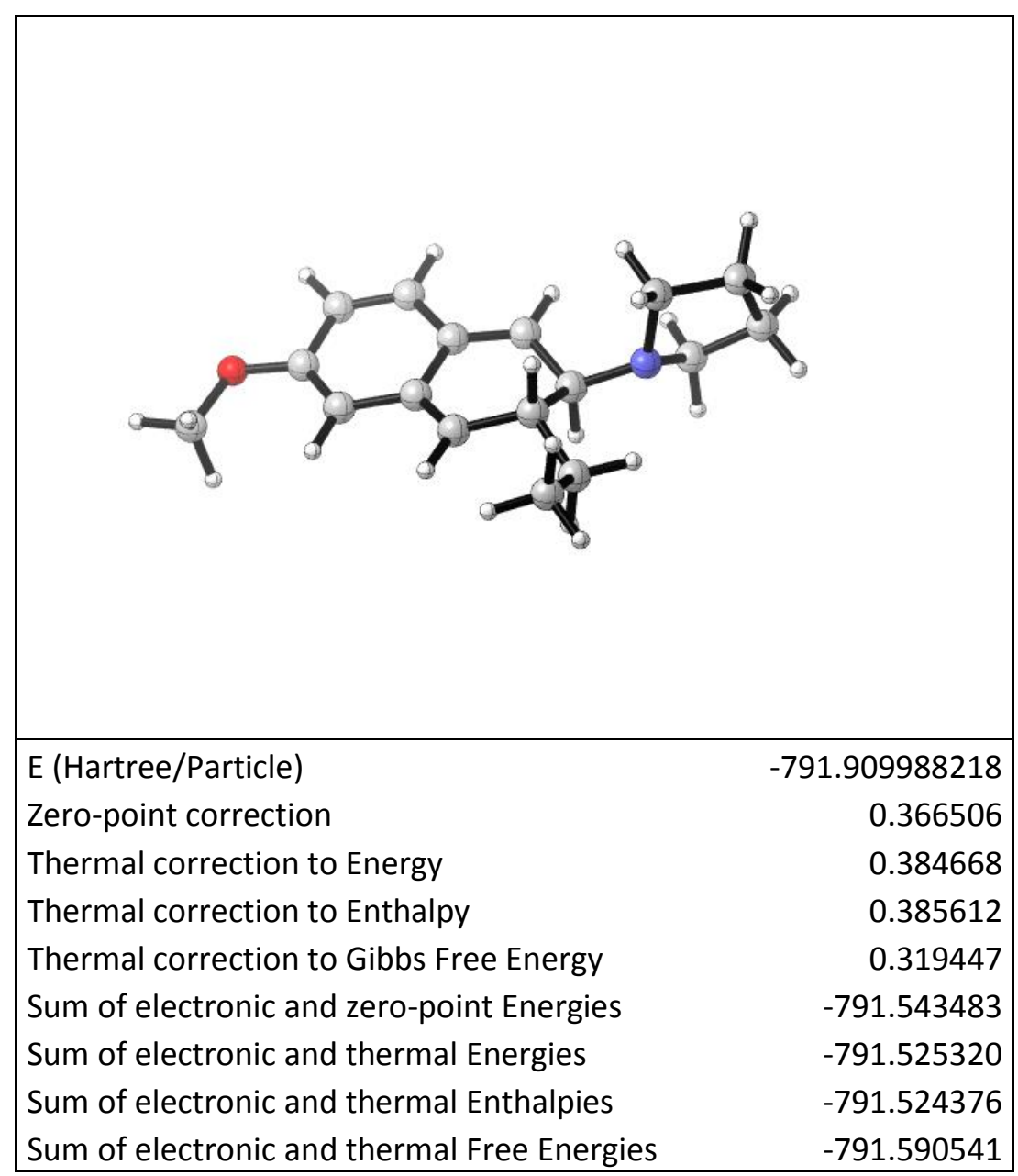




\section{References:}

(1) Ahles S.; Wegner H. A. "Recipe for the Preparation of a Bidentate Lewis Acid Catalyst", 2015. www.beilstein.tv/video/recipe-for-the-preparation-of-a-bidentate-lewis-acid-catalyst/ (accessed March 16th, 2019).

(2) Kessler, S. N.; Neuburger, M.; Wegner, H. A. Eur. J. Org. Chem. 2011, 2011, 3238-3245.

(3) Kessler, S. N.; Wegner, H. A. Org. Lett. 2012, 14, 3268-3271.

(4) Kessler, S. N.; Neuburger, M.; Wegner, H. A. J. Am. Chem. Soc. 2012, 134, 17885-17888.

(5) Allegretti, P. A.; Ferreira, E. M. Chem. Sci. 2013, 4, 1053-1058.

(6) Ahles, S.; Götz, S.; Schweighauser, L.; Brodsky, M.; Kessler, S. N.; Heindl, A. H.; Wegner, H. A. Org. Lett. 2018, 20, 7034-7038.

(7) Frisch, M. J.; Trucks, G. W.; Schlegel, H. B.; Scuseria, G. E.; Robb, M. A.; Cheeseman, J. R.; Scalmani, G.; Barone, V.; Petersson, G. A.; Nakatsuji, H.; Li, X.; Caricato, M.; Marenich, A. V.; Bloino, J.; Janesko, B. G.; Gomperts, R.; Mennucci, B.; Hratchian, H. P.; Ortiz, J. V.; Izmaylov, A. F.; Sonnenberg, J. L.; Williams; Ding, F.; Lipparini, F.; Egidi, F.; Goings, J.; Peng, B.; Petrone, A.; Henderson, T.; Ranasinghe, D.; Zakrzewski, V. G.; Gao, J.; Rega, N.; Zheng, G.; Liang, W.; Hada, M.; Ehara, M.; Toyota, K.; Fukuda, R.; Hasegawa, J.; Ishida, M.; Nakajima, T.; Honda, Y.; Kitao, O.; Nakai, H.; Vreven, T.; Throssell, K.; Montgomery Jr., J. A.; Peralta, J. E.; Ogliaro, F.; Bearpark, M. J.; Heyd, J. J.; Brothers, E. N.; Kudin, K. N.; Staroverov, V. N.; Keith, T. A.; Kobayashi, R.; Normand, J.; Raghavachari, K.; Rendell, A. P.; Burant, J. C.; Iyengar, S. S.; Tomasi, J.; Cossi, M.; Millam, J. M.; Klene, M.; Adamo, C.; Cammi, R.; Ochterski, J. W.; Martin, R. L.; Morokuma, K.; Farkas, O.; Foresman, J. B.; Fox,D. J. Gaussian 16 Rev. B.01; Wallingford, CT, 2016.

(8) Becke, A. D. J. Chem. Phys. 1993, 98, 5648-5652.

(9) Lee, C.; Yang, W.; Parr, R. G. Phys. Rev. B 1988, 37, 785-789.

(10) Grimme, S. J. Comput. Chem. 2004, 25, 1463-1473.

(11) Caricato, M. J. Chem. Theory Comput. 2012, 8, 4494-4502.

(12) Legault, C. Y. CYLview; Université de Sherbrooke: Quebec, Canada, 2009. www.Cylview.org.

(13) Runge, E.; Gross, E. K. U. Phys. Rev. Lett. 1984, 52, 997-1000.

(14) Davidson, E. R. Chem. Phys. Lett. 1996, 260, 514-518.

(15) Dunning, T. H. J. Chem. Phys. 1989, 90, 1007-1023.

(16) Yanai, T.; Tew, D. P.; Handy, N. C. Chem. Phys. Lett. 2004, 393, 51-57.

(17) Trahanovsky, W. S.; Macias, J. R. J. Am. Chem. Soc. 1986, 108, 6820-6821. 